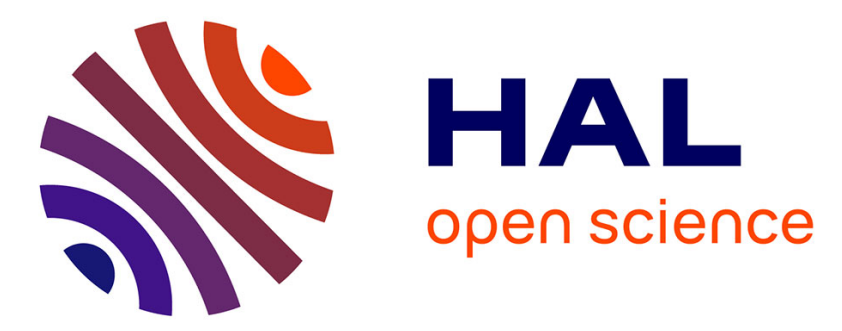

\title{
Synthesis of Novel Cyclic Nitrones withgem-Difluoroalkyl Side Chains Through Cascade Reactions
}

\author{
Ali Soulieman, Rima Ibrahim, Zeinab Barakat, Nicolas Gouault, Thierry \\ Roisnel, Joël Boustie, René Grée, Ali Hachem
}

\section{To cite this version:}

Ali Soulieman, Rima Ibrahim, Zeinab Barakat, Nicolas Gouault, Thierry Roisnel, et al.. Synthesis of Novel Cyclic Nitrones withgem-Difluoroalkyl Side Chains Through Cascade Reactions. European Journal of Organic Chemistry, 2020, 2020 (35), pp.5741-5751. 10.1002/ejoc.202000972 . hal-02957740

\section{HAL Id: hal-02957740 \\ https://hal.science/hal-02957740}

Submitted on 8 Oct 2020

HAL is a multi-disciplinary open access archive for the deposit and dissemination of scientific research documents, whether they are published or not. The documents may come from teaching and research institutions in France or abroad, or from public or private research centers.
L'archive ouverte pluridisciplinaire HAL, est destinée au dépôt et à la diffusion de documents scientifiques de niveau recherche, publiés ou non, émanant des établissements d'enseignement et de recherche français ou étrangers, des laboratoires publics ou privés. 
WILEY-VCH

\title{
Synthesis of novel cyclic nitrones with gem-difluoroalkyl side chains through cascade reactions
}

\author{
Ali Souleiman ${ }^{[a, b]}$, Rima Ibrahim ${ }^{[a]}$, Zeinab Barakat ${ }^{[a]}$, Nicolas Gouault ${ }^{[b]}$, Thierry Roisnel ${ }^{[b]}$, Joel \\ Boustie $^{[b]}$, René Grée*[b] Ali Hachem*[a]
}
[a] Lebanese University, Faculty of Sciences (I)
Laboratory for Medicinal Chemistry and Natural Products, and PRASE-EDST, Hadath, Lebanon ahachem@ul.edu.lb
[b] Univ Rennes, CNRS (Institut for Chemical Sciences in Rennes), UMR 6226, 35000 Rennes, France rene.gree@univ-rennes1.fr

Supporting information for this article is given via a link at the end of the document.

\begin{abstract}
New five-membered cyclic nitrones with gem-difluoroalkyl groups in $\gamma$-position have been prepared by a 3-step sequence starting from propargylic alcohols. This domino process involves a base-mediated isomerization reaction to enones, which are trapped in situ by nitroalkane anions. In a final step, starting from these key precursors, a reduction-cyclization process affords the target molecules. Mono- and bicyclic nitrones have been prepared by this route which allows, as well, the synthesis of nitrones with functional groups in terminal position of the side chain.
\end{abstract}

\section{Introduction}

Nitrones are presently the most used spin traps to characterize free radicals in biological systems, ${ }^{[1-5]}$ with Phenyl- $N$-tertiobutyl Nitrone (PBN) and Dimethyl-Pyrrolidine-N-oxide (DMPO, Figure 1) being the most representative examples for such uses. However, due to the variety of radical types to be studied with large differences in physical, chemical and biological properties, there is a constant need to design and develop novel nitrones. ${ }^{[6]}$ Further, nitrones have been employed also as therapeutic agents in the treatment of diseases related to oxidative stress such as neurodegeneration, ${ }^{[7,8]}$ ischemic stroke, ${ }^{[9,10]}$ cardiovascular diseases, ${ }^{[11]}$ and cancer. ${ }^{[12-15]}$ In addition, nitrones are valuable intermediates in organic synthesis affording efficiently various types of useful heterocyclic, as well as acyclic molecules. ${ }^{[16]}$ On the other hand, it is well recognized that introduction of fluorine in organic molecules can be used for a fine tuning of their physical, chemical and biological properties. ${ }^{[17]}$ Relatively few fluorinated nitrones have been described to date, mostly with fluorine atoms on the phenyl group of $\mathrm{PNB},{ }^{[18]}$ or with $\mathrm{CF}_{3}$ or $\mathrm{CHF}_{2}$ groups on the nitrone. ${ }^{[19]}$ In the case of fluorinated nitrones, ${ }^{19} \mathrm{~F}$ NMR could be also a very useful tool for monitoring biophysical and biochemical studies. ${ }^{[20]}$ Therefore, the goal of this paper is to describe short and efficient syntheses of new nitrones bearing gem-difluoroalkyl side chains in $\mathrm{y}$-position. More precisely, we will first develop our strategy for the preparation of type $\boldsymbol{A}$ model nitrones (Figure 1). Then, we will extend it to molecules $B$ with functional groups in terminal position of the alkyl chains. Such groups (ester, azide, alcohol, tosylate, and protected alcohol) could be of much use to adjust the physicochemical and biological properties of these nitrones. Further, these groups could be also employed to graft easily various types of labels eventually required for in depth in vivo biological studies. Finally, starting from the same intermediates, we will report the preparation of the

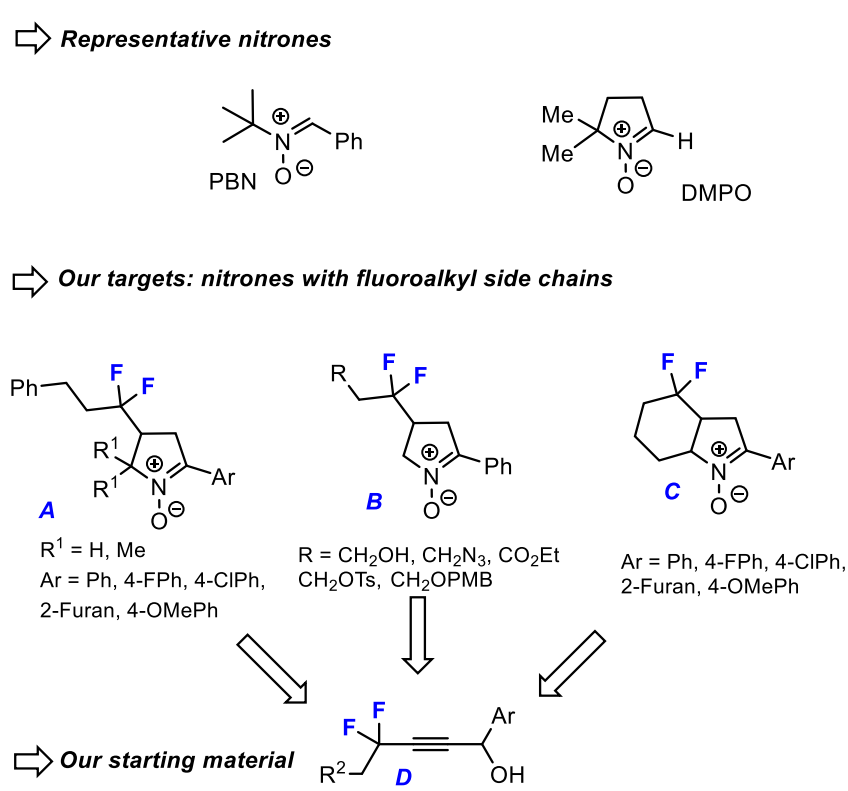

Figure 1. Our Target molecules starting from gem-difluorinated propargylic alcohols D.

bicyclic analogues $C$. All these new nitrones will be prepared in short sequences (2-3 steps) from the easily available type- $\boldsymbol{D}$ gemdifluoroalkyl propargylic alcohols. ${ }^{[21]}$

\section{Results and Discussion}

In order to prepare the first series of representative nitrones, we started from the known ${ }^{[21,22]} \mathrm{gem}$-difluoro propargylic derivative 1. After reaction with $n$-BuLi at $-90{ }^{\circ} \mathrm{C}$, followed by addition of different aldehydes 2a-e, the gem-difluorinated alcohols 3a-e were obtained (Scheme 1, Table 1). The reactions with nitromethane, or 2-nitropropane, in the presence of DBU afforded in fair to good yields the nitro intermediates 4a-e and 5a-e respectively. In agreement with previous results, the first step of this domino process is likely the base-mediated isomerization of the propargylic alcohols into the corresponding (non-isolated) enones 3'a-e which are trapped in situ by the nitroalkane anions to give the desired molecules 4 or 5 . [22] 


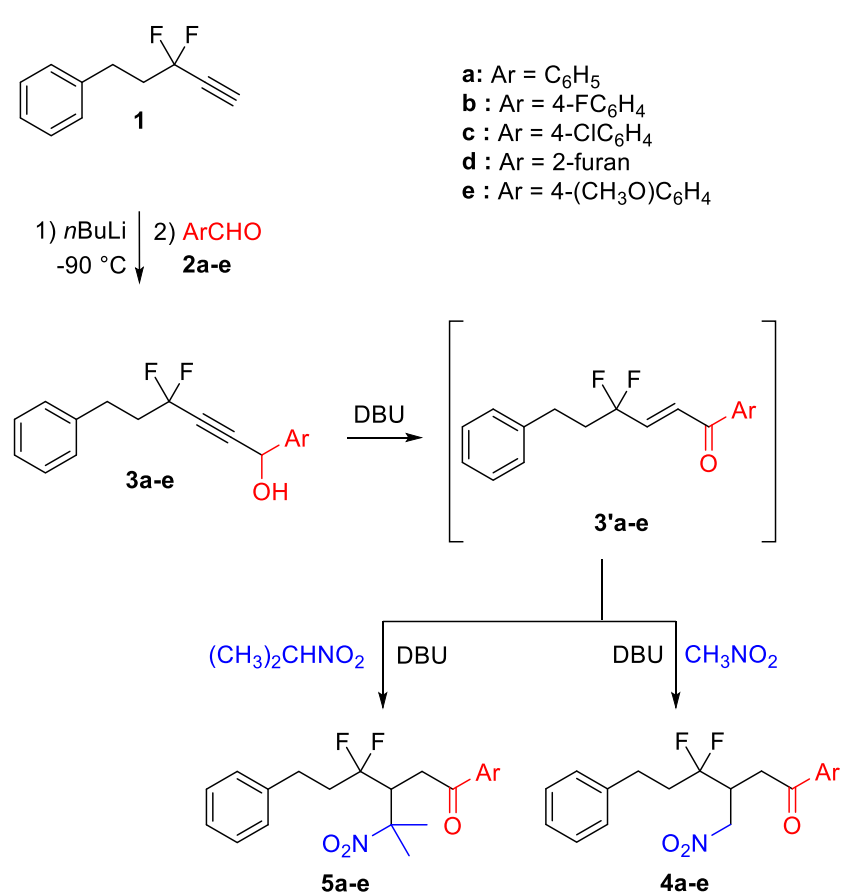

Scheme 1. The synthesis of nitro intermediates $4 a-e$ and $5 a-e$

Table 1. The synthesis of nitro intermediates 4 a-e and 5 a-e

\begin{tabular}{|c|c|c|c|c|c|}
\hline \multirow[b]{2}{*}{ Entry } & \multirow[b]{2}{*}{ SM } & \multirow[b]{2}{*}{$\mathrm{Ar}$} & \multicolumn{3}{|c|}{ Yield (\%) $)^{[a]}$} \\
\hline & & & 3 & 4 & 5 \\
\hline 1 & $2 a$ & $\mathrm{Ph}$ & $3 a: 73^{[b]}$ & $\mathbf{4 a}: 72$ & 5a : 69 \\
\hline 2 & $2 b$ & $4-\mathrm{FC}_{6} \mathrm{H}_{4}$ & $3 b: 81$ & 4b : 74 & 5b : 69 \\
\hline 3 & 2c & $4-\mathrm{ClC}_{6} \mathrm{H}_{4}$ & $3 c: 60$ & 4c: 69 & $5 c: 73$ \\
\hline 4 & $2 d$ & 2-furan & 3d : 64 & 4d : 71 & 5d: 72 \\
\hline 5 & $2 e$ & 4- $\left(\mathrm{CH}_{3} \mathrm{O}\right) \mathrm{C}_{6} \mathrm{H}_{4}$ & $3 e: 67$ & $\mathbf{4 e}: 76$ & 5e : 60 \\
\hline
\end{tabular}

[a] Isolated yields; [b] Reported previously. ${ }^{[23]}$

Several attempts were done to reduce the nitro group in 4 a using $\mathrm{Pd}(\mathrm{OH})_{2}$ and $\mathrm{H}_{2}$ gas to afford the nitrone $6 \mathbf{a}$ but unfortunately the yield was very low (15\%). Similarly, $\mathrm{NiCl}_{2} \cdot 6 \mathrm{H}_{2} \mathrm{O}$ in the presence of $\mathrm{NaBH}_{4}$ gave a poor yield (30\%), and even lower yield (10\%) was obtained by using ammonium formate in the presence of $\mathrm{Pd} / \mathrm{C}$ at $60^{\circ} \mathrm{C}$. However, upon using $\mathrm{Zn}$ powder in the presence of $\mathrm{NH}_{4} \mathrm{Cl}$ in water and THF at $0^{\circ} \mathrm{C}$, the nitrones $6 \mathrm{a}-\mathrm{e}$ and $7 \mathrm{a}$-e were successfully prepared, as shown in Scheme 2 and Table 2.

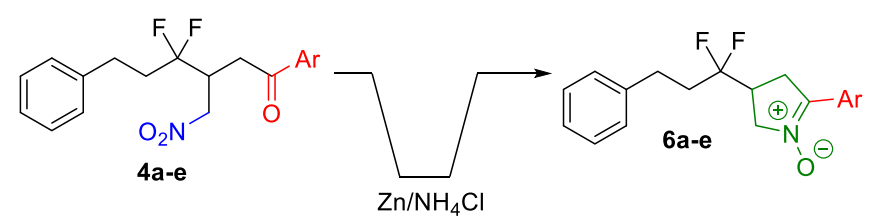<smiles>CC[C@@H](O)CCc1cccc(CCC(F)(F)C2CC(Br)N([O-])C2(C)C)c1</smiles>

Scheme 2. Synthesis of the nitrones 6a-e and 7a-e
Table 2. Synthesis of the nitrones 6a-e and 7a-e

\begin{tabular}{|c|c|c|c|c|}
\hline & & Yield (\%) ${ }^{[a]}$ & & Yield $(\%)^{[a]}$ \\
\hline Entry & SM & 6 & SM & 7 \\
\hline 1 & $4 a$ & $\mathbf{6 a}: 65$ & $5 a$ & $7 a: 90$ \\
\hline 2 & $4 b$ & $6 b: 61$ & $5 b$ & $7 b: 55$ \\
\hline 3 & $4 c$ & $6 c: 71$ & $5 c$ & $7 c: 54$ \\
\hline 4 & $4 d$ & 6d : 73 & $5 d$ & 7d : 70 \\
\hline 5 & $4 e$ & $6 e: 69$ & $5 e$ & $7 e: 85$ \\
\hline
\end{tabular}

[a] Isolated yields

All these derivatives have spectral and analytical data in agreement with the proposed structures (See experimental section and $\mathrm{SI}$ ). In addition, the structure of the nitrone $6 \mathrm{e}$ was confirmed by X-Ray analysis (Figure 2). ${ }^{24]}$

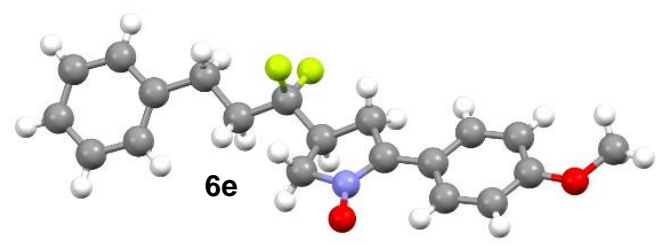

Figure 2. X-Ray structure analysis of $6 \mathbf{e}$.

Then, to expand the methodology to compounds having a functional group on the terminal position of the side chain, we started from the known ${ }^{[25]}$ ynol 8 (Scheme 3). After oxidation with Jones reagent at $0^{\circ} \mathrm{C}$ the ynone 9 was obtained in $75 \%$ yield and it was reacted with Diethylaminosulfurtrifluoride (DAST) to give the gem-difluoro propargylic intermediate 10 in $57 \%$ yield. The same sequence of reactions as before allowed us to prepare the alcohol 11a in $95 \%$ yield, the nitromethane adduct 12a with $76 \%$ yield and finally the nitrone 13a in $77 \%$ yield. On the other hand, the deprotection of $12 \mathrm{a}$ afforded the alcohol 14a in excellent yield, and the final reduction gave in $60 \%$ yield the nitrone $15 \mathrm{a}$ with a free primary alcohol group (Scheme 3).

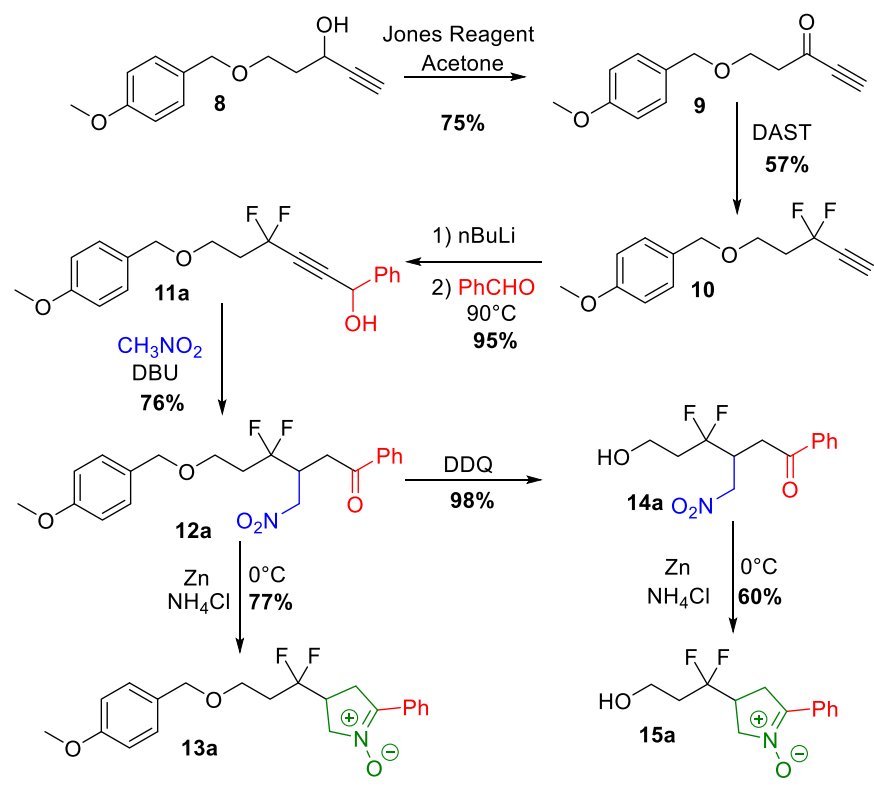

Scheme 3. Synthesis of the nitrones $13 a$ and $15 a$. 


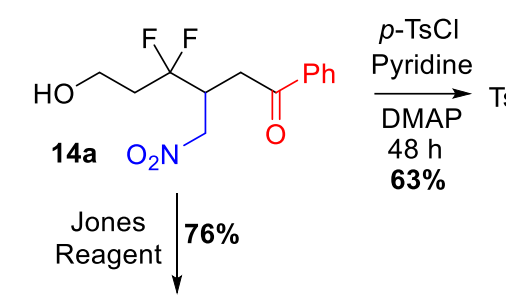

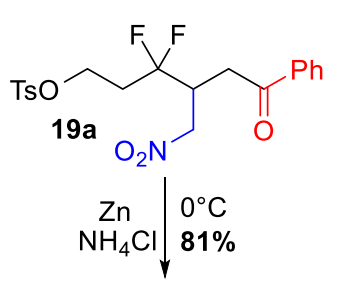<smiles>O=C(O)CC(F)(F)C(CC(=O)c1ccccc1)C[N+](=O)[O-]</smiles>

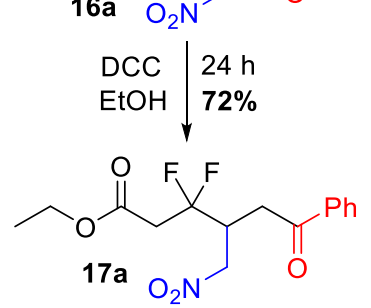
\begin{tabular}{l|l}
$\mathrm{Zn}$ & $0^{\circ} \mathrm{C}$ \\
$\mathrm{NH}_{4} \mathrm{Cl}$ & $77 \%$
\end{tabular}<smiles>CCOC(=O)CC(F)(F)C1CC(c2ccccc2)=[N+]([O-])C1</smiles>

Scheme 4. Synthesis of the nitrones, 18a, 20a, and 21a.

To complete this strategy and extend the range of functional groups in the terminal position of the side chain, alcohol 14a was first transformed into the acid $\mathbf{1 6} \mathbf{a}$ by Jones reagent. After Steglich esterification of this acid, the ester 17a was obtained and the reduction of the nitro group under the same conditions as above gave the nitrone with a terminal ester group 18a, in $42 \%$ overall yield from 14a. On the other hand, the tosylation of the alcohol 14a afforded compound 19 a which was readily reduced to the nitrone with a tosylate group $20 \mathrm{a}$ in $51 \%$ overall yield from $14 a$. Then displacing this tosylate group with sodium azide afforded the nitrone with a terminal azide 21a in $85 \%$ yield (Scheme 4). These new nitrones, with different functional groups in the terminal position of the side chain, offer many potentialities for further applications in chemistry and in studies of biological problems.

In order to expand the strategy to fused bicyclic nitrones, we started from the known, ${ }^{[22]}$ gem-difluorinated propargylic compound 22. After reaction with $n$-BuLi and different aromatic aldehydes, the propargylic alcohols 23a-e were obtained in good yields (Table 3). Substitution of the terminal primary alcohol by bromine gave the derivatives $24 a-e$ in fair to good yields. Then, reaction with nitromethane in the presence of DBU gave directly the cyclohexyl derivatives 25a-e, as single diastereoisomers (control of the crude reaction mixture using ${ }^{19} \mathrm{~F} N M R$ ). Based on our previous studies, ${ }^{[22,26]}$ in this cascade process, three steps are likely to occur sequentially: isomerization of the propargylic alcohol to the enone then nucleophilic substitution of the bromine by the nitromethane anion, and finally intramolecular Michael addition of the nitro anion onto this enone to give 25a-e as trans isomers (Scheme 5).

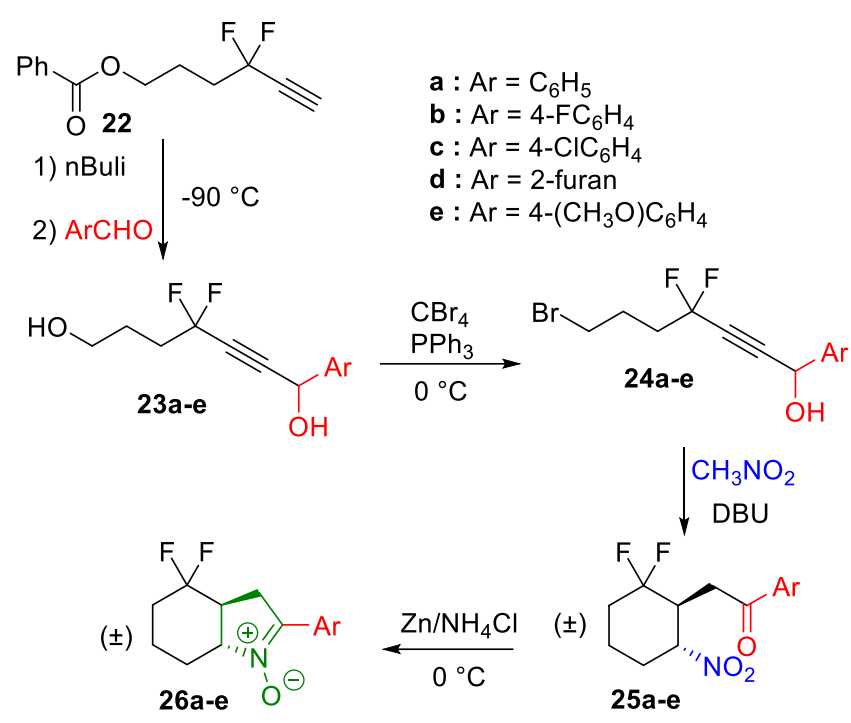

Scheme 5. Synthesis of the bicyclic nitrones 26a-e

Table 3. Synthesis of the bicyclic nitrones 26a-e

\begin{tabular}{clccccc}
\cline { 3 - 6 } & & & \multicolumn{4}{c}{ Yield (\%) $^{[\mathrm{a}]}$} \\
\hline Entry & SM & $\mathrm{Ar}$ & $\mathbf{2 3}$ & $\mathbf{2 4}$ & $\mathbf{2 5}$ & $\mathbf{2 6}$ \\
\hline 1 & 22a & $\mathrm{Ph}$ & $72^{[\mathrm{a}]}$ & $72^{[\mathrm{a}]}$ & 76 & 56 \\
2 & 22b & 4- $\mathrm{FC}_{6} \mathrm{H}_{4}$ & 70 & 88 & 66 & 70 \\
3 & 22c & 4- $\mathrm{ClC}_{6} \mathrm{H}_{4}$ & 86 & 61 & 70 & 60 \\
4 & 22d & 2-furan & 90 & 51 & 52 & 80 \\
5 & 22e & 4- $\left(\mathrm{CH}_{3} \mathrm{O}\right) \mathrm{C}_{6} \mathrm{H}_{4}$ & 95 & 77 & 49 & 75
\end{tabular}

[a] Isolated yields; [b] Reported previously [22]
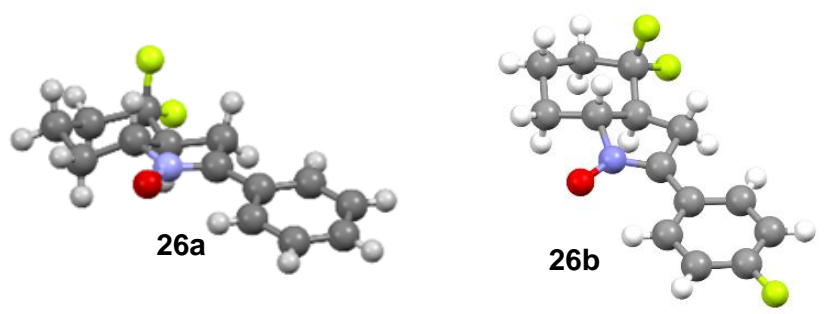

Figure 3. X-Ray structure analysis of $26 \mathrm{a}$ and $\mathbf{2 6 b}$

In a last step, the desired bicyclic nitrones were obtained using the same reduction procedure as above to give compounds 26a$\mathbf{e}$ in good yields (Table 3 ). The structure of $\mathbf{2 6 a}$ and $\mathbf{2 6} \mathbf{b}$, including the trans ring junction, were unambiguously established using $X$ Ray crystallography (Figure 3). ${ }^{[24]}$

\section{Conclusion}

Novel mono- and bicyclic nitrones with a $\mathrm{CF}_{2} \mathrm{R}$ group in $\gamma$ position have been efficiently prepared from gem-difluoro propargylic alcohols. Biological properties of these new nitrones are under active study and corresponding results will be reported in due course. 


\section{Experimental Section}

\section{General informations: see ref. 22}

\section{General procedure 1 for the synthesis of gem- difluoropropargylic alcohols 3}

To a solution of $n$-butyllithium $2.5 \mathrm{M}(1.44 \mathrm{~mL}, 3.61 \mathrm{mmol})$ in anhydrous THF (25 mL) cooled to $-90^{\circ} \mathrm{C}$, was added under nitrogen, a solution of 1 (500 mg, $2.77 \mathrm{mmol}$ ). The mixture was stirred for $30 \mathrm{~min}$ at $-90^{\circ} \mathrm{C}$. Then, aldehyde 2 (1.3 equiv) in anhydrous THF $(10 \mathrm{~mL})$ was added at $-90^{\circ} \mathrm{C}$, stirred for $45 \mathrm{~min}$, then the reaction mixture was allowed to warm to r.t. within $2 \mathrm{~h}$. The mixture was then treated with a saturated ammonium chloride solution and extracted with ethyl acetate $(3 \times 40 \mathrm{~mL})$. The combined organic phases were washed with water, dried over $\mathrm{Na}_{2} \mathrm{SO}_{4}$ and concentrated in vacuo. After purification by chromatography on silica gel, the propargylic alcohols 3 were obtained.

\section{4,4-difluoro-1-(4-fluorophenyl)-6-phenylhex-2-yn-1-ol 3b}

Pale yellow oil, yield $684 \mathrm{mg}(81 \%)$. $R_{f}=0.5$ (hexane / Ethyl Acetate: $8 / 2$ ). ${ }^{1} \mathrm{H}$ NMR $\left(300 \mathrm{MHz}, \mathrm{CDCl}_{3}\right) \delta 7.50-7.37(\mathrm{~m}, 2 \mathrm{H}), 7.32-7.10(\mathrm{~m}, 5 \mathrm{H})$, $7.09-6.97(\mathrm{~m}, 2 \mathrm{H}), 5.44\left(\mathrm{t},{ }^{3} \mathrm{JH}_{\mathrm{H}}=3.9 \mathrm{~Hz}, 1 \mathrm{H}\right), 2.90-2.76(\mathrm{~m}, 2 \mathrm{H}), 2.45$ $-2.24(\mathrm{~m}, 2 \mathrm{H}), 2.32-2.10$ (bs, $1 \mathrm{H}, \mathrm{OH}) .{ }^{13} \mathrm{C} \mathrm{NMR}\left(75 \mathrm{MHz}, \mathrm{CDCl}_{3}\right) \delta$ $163.1\left(\mathrm{~d},{ }^{1} J_{C-F}=247.9 \mathrm{~Hz}\right), 139.8,134.9,128.7,128.6\left(\mathrm{~d},{ }^{3} J_{C-F}=8.4 \mathrm{~Hz}\right)$, 128.5, 126.6, $115.9\left(\mathrm{~d},{ }^{2} J_{C-F}=21.8 \mathrm{~Hz}\right), 114.2\left(\mathrm{t},{ }^{1} J_{C-F}=233.6 \mathrm{~Hz}\right), 86.8(\mathrm{t}$, $\left.{ }^{3} J_{C-F}=6.7 \mathrm{~Hz}\right), 79.6\left(\mathrm{t},{ }^{2} J_{C-F}=41.0 \mathrm{~Hz}\right), 63.6,41.0\left(\mathrm{t},{ }^{2} J_{C-F}=26.2 \mathrm{~Hz}\right), 29.1$ $\left(\mathrm{t},{ }^{3} \mathrm{~J} C-\mathrm{F}=4.0 \mathrm{~Hz}\right) .{ }^{19} \mathrm{~F}\{\mathrm{H}\} \mathrm{NMR}\left(282 \mathrm{MHz}, \mathrm{CDCl}_{3}\right) \delta-83.75(\mathrm{~s}),-112.77$ (s). HRMS (ESI): calcd. for $[\mathrm{M}+\mathrm{Naa}]^{+}\left(\mathrm{C}_{18} \mathrm{H}_{15} \mathrm{OF}_{3} \mathrm{Na}\right)=327.0967$; found: 327.0970 (1 ppm).

\section{1-(4-chlorophenyl)-4,4-difluoro-6-phenylhex-2-yn-1-ol 3c} Yellow oil, yield $534 \mathrm{mg}(60 \%) . R_{f}=0.2$ (hexane / Ethyl Acetate: 9/1). ${ }^{1} \mathrm{H}$ $\operatorname{NMR}\left(300 \mathrm{MHz}, \mathrm{CDCl}_{3}\right) \delta 7.48-7.35(\mathrm{~m}, 4 \mathrm{H}), 7.35-7.27(\mathrm{~m}, 2 \mathrm{H}), 7.26-$ $7.16(\mathrm{~m}, 3 \mathrm{H}), 5.49(\mathrm{bs}, 1 \mathrm{H}), 2.94-2.83(\mathrm{~m}, 2 \mathrm{H}), 2.49-2.31(\mathrm{~m}, 2 \mathrm{H}), 2.27$ (bs, $1 \mathrm{H}, \mathrm{OH}) .{ }^{13} \mathrm{C} \mathrm{NMR}\left(75 \mathrm{MHz}, \mathrm{CDCl}_{3}\right) \delta 139.8,137.5,135.0,129.2$, 128.8, 128.5, 128.1, 126.6, $114.2\left(\mathrm{t},{ }^{1} J_{C-F}=233.7 \mathrm{~Hz}\right), 86.6\left(\mathrm{t},{ }^{3} J_{C-F}=6.7\right.$ $\mathrm{Hz}), 79.7\left(\mathrm{t},{ }^{2} J_{C-F}=41.0 \mathrm{~Hz}\right), 63.6,40.9\left(\mathrm{t},{ }^{2} J_{C-F}=26.1 \mathrm{~Hz}\right), 29.1\left(\mathrm{t},{ }^{3} J_{C-F}=\right.$ $4.0 \mathrm{~Hz}) .{ }^{19} \mathrm{~F}\{\mathrm{H}\} \mathrm{NMR}\{\mathrm{H}\}\left(282 \mathrm{MHz}, \mathrm{CDCl}_{3}\right) \delta-83.81$ (s). HRMS (ESI): calcd. for $[\mathrm{M}+\mathrm{Na}]^{+}\left(\mathrm{C}_{18} \mathrm{H}_{15} \mathrm{OF}_{2}{ }^{35} \mathrm{CINa}\right)=343.0671 ; 343.0672(0 \mathrm{ppm})$.

\section{4,4-difluoro-1-(furan-2-yl)-6-phenylhex-2-yn-1-ol 3d}

Black oil, yield $490 \mathrm{mg}(64 \%) . R_{f}=0.3$ (hexane / Ethyl Acetate: 8/2). ${ }^{1} \mathrm{H}$ $\operatorname{NMR}\left(300 \mathrm{MHz}, \mathrm{CDCl}_{3}\right) \delta 7.41$ (dd, $\left.{ }^{3} \mathrm{JH}_{\mathrm{H}}=1.9,{ }^{3} \mathrm{JH}_{\mathrm{H}}=0.8 \mathrm{~Hz}, 1 \mathrm{H}\right), 7.33-$ $7.24(\mathrm{~m}, 2 \mathrm{H}), 7.23-7.14(\mathrm{~m}, 3 \mathrm{H}), 6.44\left(\mathrm{dd},{ }^{3} \mathrm{~J}_{\mathrm{H}-\mathrm{H}}=3.3 \mathrm{~Hz},{ }^{3} \mathrm{JH}_{\mathrm{H}}=0.8 \mathrm{~Hz}\right.$, $1 \mathrm{H}), 6.35\left(\mathrm{dd},{ }^{3} \mathrm{~J}_{\mathrm{H}-\mathrm{H}}=3.3 \mathrm{~Hz},{ }^{3} \mathrm{~J}_{\mathrm{H}-\mathrm{H}}=1.9 \mathrm{~Hz}, 1 \mathrm{H}\right), 5.49\left(\mathrm{t},{ }^{5} \mathrm{~J}_{\mathrm{H}-\mathrm{F}}=3.9 \mathrm{~Hz}\right.$, $1 \mathrm{H}), 2.92-2.81(\mathrm{~m}, 2 \mathrm{H}), 2.68(\mathrm{bs}, 1 \mathrm{H}, \mathrm{OH}), 2.46-2.27(\mathrm{~m}, 2 \mathrm{H}) .{ }^{13} \mathrm{C} \mathrm{NMR}$ $\left(75 \mathrm{MHz}, \mathrm{CDCl}_{3}\right) \delta 151.4,143.6,139.9,128.7,128.5,126.5,114.2\left(\mathrm{t},{ }^{1} \mathrm{~J}_{C}\right.$ $F=233.7 \mathrm{~Hz}), 110.7,108.6,84.7\left(\mathrm{t},{ }^{3} J_{C-F}=6.8 \mathrm{~Hz}\right), 78.6\left(\mathrm{t},{ }^{2} J_{C-F}=41.1\right.$ $\mathrm{Hz}), 57.9\left(\mathrm{t},{ }^{4} J_{C-F}=1.8 \mathrm{~Hz}\right), 41.0\left(\mathrm{t},{ }^{2} J_{C-F}=26.0 \mathrm{~Hz}\right), 29.1\left(\mathrm{t},{ }^{3} J_{C-F}=4.0 \mathrm{~Hz}\right)$. ${ }^{19} \mathrm{~F}\{\mathrm{H}\}$ NMR (282 MHz, $\left.\mathrm{CDCl}_{3}\right) \delta-84.02$ (s). HRMS (ESI): calcd. for $[\mathrm{M}+\mathrm{Naa}]^{+}\left(\mathrm{C}_{16} \mathrm{H}_{14} \mathrm{O}_{2} \mathrm{~F}_{2} \mathrm{Na}\right)=299.0854$; found: 299.0857 (1 ppm).

\section{4,4-difluoro-1-(4-methoxyphenyl)-6-phenylhex-2-yn-1-ol 3e}

Pale yellow oil, yield $588 \mathrm{mg}(67 \%)$. R $\mathrm{f}=0.2$ (Petroleum Ether / Ethyl Acetate: 8/2). ${ }^{1} \mathrm{H}$ NMR $\left(300 \mathrm{MHz}, \mathrm{CDCl}_{3}\right) \delta 7.47-7.40(\mathrm{~m}, 2 \mathrm{H}), 7.35-$ $7.27(\mathrm{~m}, 2 \mathrm{H}), 7.26-7.17(\mathrm{~m}, 3 \mathrm{H}), 6.96-6.89(\mathrm{~m}, 2 \mathrm{H}), 5.47\left(\mathrm{t},{ }^{5} \mathrm{~J}_{\mathrm{H}-\mathrm{F}}=3.9\right.$ $\mathrm{Hz}, 1 \mathrm{H}), 3.82(\mathrm{~s}, 3 \mathrm{H}), 2.92-2.85(\mathrm{~m}, 2 \mathrm{H}), 2.48-2.31(\mathrm{~m}, 2 \mathrm{H}), 2.28(\mathrm{bs}$, 1H). ${ }^{13} \mathrm{C}$ NMR $\left(75 \mathrm{MHz}, \mathrm{CDCl}_{3}\right) \delta 160.1,139.9,131.4\left(\mathrm{t},{ }^{5} \mathrm{JC}-\mathrm{F}=1.6 \mathrm{~Hz}\right)$, 128.7, 128.5, 128.2, 126.5, 114.3, $114.2\left(\mathrm{t},{ }^{1} J_{C-F}=233.4 \mathrm{~Hz}\right), 87.3\left(\mathrm{t},{ }^{3} J_{C-F}\right.$ $=6.8 \mathrm{~Hz}), 79.2\left(\mathrm{t},{ }^{2} J_{C-F}=40.9 \mathrm{~Hz}\right), 63.9\left(\mathrm{t},{ }^{4} J_{C-F}=2.0 \mathrm{~Hz}\right), 55.5,41.0(\mathrm{t}$, $\left.{ }^{2} J_{C-F}=26.2 \mathrm{~Hz}\right), 29.1\left(\mathrm{t},{ }^{3} J_{C-F}=4.0 \mathrm{~Hz}\right) \cdot{ }^{19} \mathrm{~F}\{\mathrm{H}\} \mathrm{NMR}\left(376 \mathrm{MHz}, \mathrm{CDCl}_{3}\right) \delta$ -83.61 (s). HRMS (ESI): calcd. for $[\mathrm{M}+\mathrm{Na}]^{+}\left(\mathrm{C}_{19} \mathrm{H}_{18} \mathrm{O}_{2} \mathrm{~F}_{2} \mathrm{Na}\right)=339.1167$; found: 339.1169 (1 ppm).
General procedure 2 for the synthesis of $\mathrm{gem}$-difluoronitroketones 4 and 5

Representative procedure: Synthesis of 4,4-difluoro-3-(nitromethyl)1,6-diphenylhexan-1-one 4a

To the difluoropropargylic alcohol $3 a(150 \mathrm{mg}, 0.52 \mathrm{mmol})$ was added nitromethane ( $56 \mu \mathrm{L}, 1.04 \mathrm{mmol})$ and DBU $(310 \mu \mathrm{l}, 2.10 \mathrm{mmol})$ in THF (3 $\mathrm{ml})$. The reaction mixture was stirred at room temperature overnight. At the end saturated $\mathrm{NH}_{4} \mathrm{Cl}(10 \mathrm{~mL})$ was added and the reaction mixture was extracted with ethyl acetate $(3 \times 20 \mathrm{~mL})$. The organic layers were separated, washed with water ( $1 \times 10 \mathrm{~mL})$, dried over $\mathrm{Na}_{2} \mathrm{SO}_{4}$ and concentrated under vacuum. After purification by chromatography on silica gel, $4 a(131 \mathrm{mg}$, $72 \%$ ) was obtained as white solid. $R_{f}=0.2$ (hexane / Ethyl Acetate: 9/1). $\mathrm{mp}=102{ }^{\circ} \mathrm{C} .{ }^{1} \mathrm{H}$ NMR $\left(300 \mathrm{MHz}, \mathrm{CDCl}_{3}\right) \delta 8.01-7.92(\mathrm{~m}, 2 \mathrm{H}), 7.67-$ $7.57(\mathrm{~m}, 1 \mathrm{H}), 7.54-7.44(\mathrm{~m}, 2 \mathrm{H}), 7.34-7.15(\mathrm{~m}, 5 \mathrm{H}), 4.74\left(\mathrm{dd},{ }^{2} \mathrm{~J}_{\mathrm{H}-\mathrm{H}}=\right.$ $\left.13.6 \mathrm{~Hz},{ }^{3} \mathrm{JH}_{\mathrm{H}-\mathrm{H}}=6.1 \mathrm{~Hz}, 1 \mathrm{H}\right), 4.52\left(\mathrm{dd},{ }^{2} \mathrm{JH}_{\mathrm{H} H}=13.6 \mathrm{~Hz},{ }^{2} \mathrm{JH}-\mathrm{H}=5.5 \mathrm{~Hz}, 1 \mathrm{H}\right)$, $3.83-3.62(\mathrm{~m}, 1 \mathrm{H}), 3.41\left(\mathrm{dd},{ }^{2} \mathrm{~J}_{\mathrm{H}-\mathrm{H}}=18.5 \mathrm{~Hz},{ }^{2} \mathrm{~J}_{\mathrm{H}-\mathrm{H}}=4.6 \mathrm{~Hz}, 1 \mathrm{H}\right), 3.24$ $\left(\mathrm{dd},{ }^{2} \mathrm{~J}_{\mathrm{H}-\mathrm{H}}=18.5 \mathrm{~Hz},{ }^{2} \mathrm{~J}_{\mathrm{H}-\mathrm{H}}=8.1 \mathrm{~Hz}, 1 \mathrm{H}\right), 2.94-2.82(\mathrm{~m}, 2 \mathrm{H}), 2.33-2.10$ (m, 2H). ${ }^{13} \mathrm{C} \mathrm{NMR}\left(75 \mathrm{MHz}, \mathrm{CDCl}_{3}\right) \delta 196.1,140.0,136.1,134.0,129.0$, 128.8, 128.4, 128.3, 126.6, $124.1\left(\mathrm{t},{ }^{1} J_{C-F}=245.7 \mathrm{~Hz}\right), 73.9\left(\mathrm{t},{ }^{3} J_{C-F}=4.8\right.$ $\mathrm{Hz}), 39.3\left(\mathrm{t},{ }^{2} J_{C-F}=24.5 \mathrm{~Hz}\right), 37.0\left(\mathrm{t},{ }^{2} J_{C-F}=24.6 \mathrm{~Hz}\right), 35.4\left(\mathrm{t},{ }^{3} J_{C-F}=3.6\right.$ $\mathrm{Hz}), 28.0\left(\mathrm{t},{ }^{3} J_{C-F}=5.1 \mathrm{~Hz}\right) .{ }^{19} \mathrm{~F}\{\mathrm{H}\} \mathrm{NMR}\left(282 \mathrm{MHz}, \mathrm{CDCl}_{3}\right) \delta-102.95(\mathrm{AB}$ system, $\left.{ }^{2} J_{F-F}=248.9 \mathrm{~Hz}\right),-104.05\left(\mathrm{AB}\right.$ system, $\left.{ }^{2} J_{F-F}=248.9 \mathrm{~Hz}\right)$. HRMS (ESI): calcd. for $[\mathrm{M}+\mathrm{Na}]^{+}\left(\mathrm{C}_{19} \mathrm{H}_{19} \mathrm{NO}_{3} \mathrm{~F}_{2} \mathrm{Na}\right)=370.1225$; found: 370.1230 (1 ppm).

\section{4,4-difluoro-1-(4-fluorophenyl)-3-(nitromethyl)-6-phenylhexan-1-one} $4 \mathrm{~b}$

Yellow solid, yield $133 \mathrm{mg}(74 \%)$ from $150 \mathrm{mg}(0.49 \mathrm{mmol})$ of $3 \mathbf{b} . \mathrm{R}_{\mathrm{f}}=0.4$ (hexane / Ethyl Acetate: 9/1). $\mathrm{mp}=129^{\circ} \mathrm{C} .{ }^{1} \mathrm{H} \mathrm{NMR}\left(300 \mathrm{MHz}, \mathrm{CDCl}_{3}\right) \delta$ $8.15-7.98(\mathrm{~m}, 2 \mathrm{H}), 7.48-7.08(\mathrm{~m}, 7 \mathrm{H}), 4.80\left(\mathrm{dd},{ }^{2} \mathrm{~J}_{\mathrm{H} H}=13.6 \mathrm{~Hz},{ }^{3} \mathrm{JH}_{\mathrm{H} H}=\right.$ $5.9 \mathrm{~Hz}, 1 \mathrm{H}), 4.58\left(\mathrm{dd},{ }^{2} \mathrm{JH}_{\mathrm{H}}=13.6 \mathrm{~Hz},{ }^{3} \mathrm{JH}-\mathrm{H}=5.6 \mathrm{~Hz}, 1 \mathrm{H}\right), 3.87-3.63(\mathrm{~m}$, $1 \mathrm{H}), 3.45\left(\mathrm{dd},{ }^{2} \mathrm{~J}_{\mathrm{H}-\mathrm{H}}=18.5 \mathrm{~Hz},{ }^{3} \mathrm{~J}_{\mathrm{H}-\mathrm{H}}=4.6 \mathrm{~Hz}, 1 \mathrm{H}\right), 3.29\left(\mathrm{dd},{ }^{2} \mathrm{~J}_{\mathrm{H}-\mathrm{H}}=18.4 \mathrm{~Hz}\right.$, $\left.{ }^{3} \mathrm{JH}_{\mathrm{H}-\mathrm{H}}=8.0 \mathrm{~Hz}, 1 \mathrm{H}\right), 3.09-2.81(\mathrm{~m}, 2 \mathrm{H}), 2.39-2.16(\mathrm{~m}, 2 \mathrm{H}) .{ }^{13} \mathrm{C} \mathrm{NMR}(75$ $\left.\mathrm{MHz}, \mathrm{CDCl}_{3}\right) \delta 194.5,166.3\left(\mathrm{~d},{ }^{1} \mathrm{JC}_{\mathrm{F}-\mathrm{F}}=256.3 \mathrm{~Hz}\right), 139.9,132.5\left(\mathrm{~d},{ }^{4} \mathrm{JC}_{\mathrm{F}} \mathrm{F}=\right.$ $3.0 \mathrm{~Hz}$ ), 131.0 (d, $\left.{ }^{3} J_{C-F}=9.5 \mathrm{~Hz}\right), 128.8,128.4,124.0\left(\mathrm{t},{ }^{1} J_{C-F}=245.8 \mathrm{~Hz}\right)$, $116.2\left(\mathrm{~d},{ }^{2} J_{C-F}=22.1 \mathrm{~Hz}\right), 73.8\left(\mathrm{t},{ }^{3} J_{C-F}=4.9 \mathrm{~Hz}\right), 39.3\left(\mathrm{t},{ }^{2} J_{C-F}=24.5 \mathrm{~Hz}\right)$, $36.9\left(\mathrm{t},{ }^{2} J_{C-F}=24.6 \mathrm{~Hz}\right), 35.2\left(\mathrm{t},{ }^{3} J_{C-F}=3.7 \mathrm{~Hz}\right), 28.0\left(\mathrm{t},{ }^{3} J_{C-F}=5.1 \mathrm{~Hz}\right) .{ }^{19} \mathrm{~F}$ $\{\mathrm{H}\} \operatorname{NMR}\left(282 \mathrm{MHz}, \mathrm{CDCl}_{3}\right) \delta-103.00\left(\mathrm{AB}\right.$ system, $\left.{ }^{2} J_{F-F}=248.8 \mathrm{~Hz}\right)$, 103.52 (s), -139.95 (AB system, ${ }^{2} J_{F-F}=248.8 \mathrm{~Hz}$ ). HRMS (ESI): calcd. for $[\mathrm{M}+\mathrm{Na}]^{+}\left(\mathrm{C}_{19} \mathrm{H}_{18} \mathrm{NO}_{3} \mathrm{~F}_{3} \mathrm{Na}\right)=388.1131$; found: 388.1131 (0 ppm).

1-(4-chlorophenyl)-4,4-difluoro-3-(nitromethyl)-6-phenylhexan-1-one $4 c$

White solid, yield $123 \mathrm{mg}(69 \%)$ from $150 \mathrm{mg}(0.47 \mathrm{mmol})$ of $3 \mathrm{c} . \mathrm{R}_{\mathrm{f}}=0.3$ (hexane / Ethyl Acetate: 9/1). $\mathrm{mp}=137^{\circ} \mathrm{C} .{ }^{1} \mathrm{H}$ NMR $\left(300 \mathrm{MHz}, \mathrm{CDCl}_{3}\right) \delta$ $7.94-7.86(\mathrm{~m}, 2 \mathrm{H}), 7.52-7.42(\mathrm{~m}, 2 \mathrm{H}), 7.34-7.12(\mathrm{~m}, 5 \mathrm{H}), 4.73$ (dd, $\left.{ }^{2} \mathrm{JH}-\mathrm{H}=13.6 \mathrm{~Hz},{ }^{3} \mathrm{JH}_{\mathrm{H}-\mathrm{H}}=5.9 \mathrm{~Hz}, 1 \mathrm{H}\right), 4.51\left(\mathrm{dd},{ }^{2} \mathrm{~J}_{\mathrm{H}-\mathrm{H}}=13.6,{ }^{3} \mathrm{~J}_{\mathrm{H}-\mathrm{H}}=5.6 \mathrm{~Hz}\right.$, $1 \mathrm{H}), 3.79-3.54(\mathrm{~m}, 1 \mathrm{H}), 3.37\left(\mathrm{dd},{ }^{2} J_{H-H}=18.5 \mathrm{~Hz},{ }^{3} \mathrm{~J}_{\mathrm{H}-\mathrm{H}}=4.6 \mathrm{~Hz}, 1 \mathrm{H}\right)$, $3.22\left(\mathrm{dd},{ }^{2} \mathrm{~J}_{\mathrm{H}-\mathrm{H}}=18.5 \mathrm{~Hz},{ }^{3} \mathrm{~J}_{\mathrm{H}-\mathrm{H}}=8.0 \mathrm{~Hz}, 1 \mathrm{H}\right), 2.93-2.77(\mathrm{~m}, 2 \mathrm{H}), 2.33-$ $2.09(\mathrm{~m}, 2 \mathrm{H}) .{ }^{13} \mathrm{C} \mathrm{NMR}\left(75 \mathrm{MHz}, \mathrm{CDCl}_{3}\right) \delta 195.0,140.6,139.9,134.4$, 129.7, 129.3, 128.8, 126.6, $124.0\left(\mathrm{t},{ }^{1} J_{C-F}=245.7 \mathrm{~Hz}\right), 73.8\left(\mathrm{t},{ }^{3} J_{C-F}=4.9\right.$ $\mathrm{Hz}), 39.3\left(\mathrm{t},{ }^{2} J_{C-F}=24.5 \mathrm{~Hz}\right), 36.9\left(\mathrm{t},{ }^{2} J_{C-F}=24.7 \mathrm{~Hz}\right), 35.3\left(\mathrm{t},{ }^{3} J_{C-F}=3.6\right.$ $\mathrm{Hz}), 28.0\left(\mathrm{dd},{ }^{3} J_{C-F}=5.8 \mathrm{~Hz},{ }^{3} J_{C-F}=5.0 \mathrm{~Hz}\right) .{ }^{19} \mathrm{~F}\{\mathrm{H}\} \mathrm{NMR}(471 \mathrm{MHz}$, $\left.\mathrm{CDCl}_{3}\right) \delta-103.0\left(\mathrm{AB}\right.$ system, $\left.{ }^{2} \mathrm{JF}_{F-F}=249.2 \mathrm{~Hz}\right),-103.6\left(\mathrm{AB}\right.$ system, ${ }^{2} J_{F-F}=$ $249.2 \mathrm{~Hz})$. HRMS (ESI): calcd. for $[\mathrm{M}+\mathrm{Na}]^{+}\left(\mathrm{C}_{19} \mathrm{H}_{18} \mathrm{NO}_{3} \mathrm{~F}_{2}{ }^{35} \mathrm{CINa}\right)=$ 404.0835; found: 404.0832 (1 ppm).

\section{4,4-difluoro-1-(furan-2-yl)-3-(nitromethyl)-6-phenylhexan-1-one 4d}

Black solid, yield $130 \mathrm{mg}(71 \%)$ from $150 \mathrm{mg}(0.54 \mathrm{mmol})$ of $3 \mathrm{~d}$. $\mathrm{R}_{\mathrm{f}}=0.4$ (hexane / Ethyl Acetate: 8/2). $\mathrm{mp}=79^{\circ} \mathrm{C} .{ }^{1} \mathrm{H} \mathrm{NMR}\left(300 \mathrm{MHz}, \mathrm{CDCl}_{3}\right) \delta$ $7.68\left(\mathrm{dd},{ }^{3} \mathrm{~J}_{\mathrm{H}-\mathrm{H}}=1.7 \mathrm{~Hz},{ }^{4} \mathrm{~J}_{\mathrm{H}-\mathrm{H}}=0.8 \mathrm{~Hz}, 1 \mathrm{H}\right), 7.46-7.15(\mathrm{~m}, 6 \mathrm{H}), 6.65$ (dd, $\left.{ }^{3} J_{H-H}=3.6 \mathrm{~Hz},{ }^{3} J_{H-H}=1.7 \mathrm{~Hz}, 1 \mathrm{H}\right), 4.80\left(\mathrm{dd},{ }^{2} \mathrm{~J}_{\mathrm{H}-\mathrm{H}}=13.7 \mathrm{~Hz},{ }^{3} \mathrm{~J}_{\mathrm{H}-\mathrm{H}}=6.1\right.$ $\mathrm{Hz}, 1 \mathrm{H}), 4.59\left(\mathrm{dd},{ }^{2} \mathrm{~J}_{\mathrm{H}-\mathrm{H}}=13.7 \mathrm{~Hz},{ }^{3} \mathrm{~J}_{\mathrm{H}-\mathrm{H}}=5.7 \mathrm{~Hz}, 1 \mathrm{H}\right), 3.90-3.59(\mathrm{~m}, 1 \mathrm{H})$, 
$3.35\left(\mathrm{dd},{ }^{2} J_{H-H}=18.1 \mathrm{~Hz},{ }^{3} \mathrm{JH}_{\mathrm{H}}=4.9 \mathrm{~Hz}, 1 \mathrm{H}\right), 3.16\left(\mathrm{dd},{ }^{2} \mathrm{~J}_{\mathrm{H}-\mathrm{H}}=18.1 \mathrm{~Hz}\right.$, $\left.{ }^{3} \mathrm{JH}_{\mathrm{H}-\mathrm{H}}=7.9 \mathrm{~Hz}, 1 \mathrm{H}\right), 3.00-2.75(\mathrm{~m}, 2 \mathrm{H}), 2.46-2.14(\mathrm{~m}, 2 \mathrm{H}) .{ }^{13} \mathrm{C}$ NMR $(75$ $\left.\mathrm{MHz}, \mathrm{CDCl}_{3}\right) \delta 185.1,152.1,147.1,140.0,128.8,128.4,126.5,124.0(\mathrm{t}$, $\left.{ }^{1} J_{C-F}=245.8 \mathrm{~Hz}\right), 118.0,112.8,73.7\left(\mathrm{t},{ }^{3} J_{C-F}=4.9 \mathrm{~Hz}\right), 39.0\left(\mathrm{t},{ }^{2} J_{C-F}=24.6\right.$ $\mathrm{Hz}), 36.8\left(\mathrm{t},{ }^{2} J_{C-F}=24.6 \mathrm{~Hz}\right), 35.1\left(\mathrm{t},{ }^{3} J_{C-F}=3.8 \mathrm{~Hz}\right), 27.9\left(\mathrm{t},{ }^{3} J_{C-F}=5.1 \mathrm{~Hz}\right)$. ${ }^{19} \mathrm{~F}\{\mathrm{H}\} \mathrm{NMR}\left(282 \mathrm{MHz}, \mathrm{CDCl}_{3}\right) \delta-102.94\left(\mathrm{AB}\right.$ system, $\left.{ }^{2} \mathrm{~J}-\mathrm{F}=248.6 \mathrm{~Hz}\right)$, 104.39 (AB system, ${ }^{2} J_{F-F}=248.6 \mathrm{~Hz}$ ). HRMS (ESI): calcd. for $[\mathrm{M}+\mathrm{Na}]^{+}$ $\left(\mathrm{C}_{17} \mathrm{H}_{17} \mathrm{NO}_{4} \mathrm{~F}_{2} \mathrm{Na}\right)=360.1018$; found: $360.1019(0 \mathrm{ppm})$.

\section{4,4-difluoro-1-(4-methoxyphenyl)-3-(nitromethyl)-6-phenylhexan-1-} one $4 \mathrm{e}$

White solid, yield $130 \mathrm{mg}(76 \%)$ from $150 \mathrm{mg}(0.47 \mathrm{mmol})$ of $3 e . R_{f}=0.5$ (Petroleum Ether / Ethyl Acetate: 8/2). $\mathrm{mp}=107^{\circ} \mathrm{C} .{ }^{1} \mathrm{H}$ NMR $(300 \mathrm{MHz}$, $\left.\mathrm{CDCl}_{3}\right) \delta 8.03-7.87(\mathrm{~m}, 2 \mathrm{H}), 7.42-7.10(\mathrm{~m}, 5 \mathrm{H}), 7.01-6.91(\mathrm{~m}, 2 \mathrm{H})$, $4.73\left(\mathrm{dd},{ }^{2} \mathrm{JH}_{\mathrm{H}}=13.6 \mathrm{~Hz},{ }^{3} \mathrm{JHH}_{\mathrm{H}}=6.2 \mathrm{~Hz}, 1 \mathrm{H}\right), 4.52\left(\mathrm{dd},{ }^{2} \mathrm{JHH}_{\mathrm{H}}=13.6 \mathrm{~Hz},{ }^{3} \mathrm{~J}_{\mathrm{H}-}\right.$ $H=5.3 \mathrm{~Hz}, 1 \mathrm{H}), 3.88(\mathrm{~s}, 3 \mathrm{H}), 3.80-3.62(\mathrm{~m}, 1 \mathrm{H}), 3.35\left(\mathrm{dd},{ }^{2} \mathrm{~J}_{H-H}=18.3\right.$ $\left.\mathrm{Hz},{ }^{3} \mathrm{JH}_{\mathrm{H} H}=4.5 \mathrm{~Hz}, 1 \mathrm{H}\right), 3.18\left(\mathrm{dd},{ }^{2} \mathrm{JH}-\mathrm{H}=18.3 \mathrm{~Hz},{ }^{3} \mathrm{JH}_{\mathrm{H}}=8.2 \mathrm{~Hz}, 1 \mathrm{H}\right), 2.94$ $-2.81(\mathrm{~m}, 2 \mathrm{H}), 2.32-2.09(\mathrm{~m}, 2 \mathrm{H}) .{ }^{13} \mathrm{C} \mathrm{NMR}\left(75 \mathrm{MHz}, \mathrm{CDCl}_{3}\right) \delta 194.5$, 164.2, 140.0, 130.6, 129.1, 128.8, 128.4, 126.5, $124.2\left(\mathrm{t},{ }^{1} J_{C-F}=245.7 \mathrm{~Hz}\right)$, $114.1,73.9\left(\mathrm{t},{ }^{3} J_{C-F}=4.8 \mathrm{~Hz}\right), 55.7,39.4\left(\mathrm{t},{ }^{2} J_{C-F}=24.3 \mathrm{~Hz}\right), 36.9\left(\mathrm{t},{ }^{2} J_{C-F}\right.$ $=24.6 \mathrm{~Hz}), 34.9\left(\mathrm{dd},{ }^{3} J_{C-F}=4.1,{ }^{3} J_{C-F}=3.1 \mathrm{~Hz}\right), 28.0\left(\mathrm{t},{ }^{3} J_{C-F}=5.0 \mathrm{~Hz}\right) .{ }^{19} \mathrm{~F}$ $\{\mathrm{H}\} \mathrm{NMR}\left(282 \mathrm{MHz}, \mathrm{CDCl}_{3}\right) \delta-102.83\left(\mathrm{AB}\right.$ system, $\left.{ }^{2} J_{F-F}=248.6 \mathrm{~Hz}\right)$, 104.10 (AB system, ${ }^{2} J_{F-F}=248.6 \mathrm{~Hz}$ ). HRMS (ESI): calcd. for $[\mathrm{M}+\mathrm{Na}]^{+}$ $\left(\mathrm{C}_{20} \mathrm{H}_{21} \mathrm{NO}_{4} \mathrm{~F}_{2} \mathrm{Na}\right)=400.1331$; found: $400.1333(0 \mathrm{ppm})$.

\section{4,4-difluoro-3-(2-nitropropan-2-yl)-1,6-diphenylhexan-1-one 5a}

Yellow solid, yield $136 \mathrm{mg}(69 \%)$ from $150 \mathrm{mg}(0.47 \mathrm{mmol})$ of $3 a$. $R_{f}=0.3$ (hexane / Ethyl Acetate: 9/1). $\mathrm{mp}=71^{\circ} \mathrm{C} .{ }^{1} \mathrm{H}$ NMR $\left(300 \mathrm{MHz}, \mathrm{CDCl}_{3}\right) \delta$ $8.10-7.94(\mathrm{~m}, 2 \mathrm{H}), 7.68-7.59(\mathrm{~m}, 1 \mathrm{H}), 7.58-7.42(\mathrm{~m}, 2 \mathrm{H}), 7.32-7.07$ (m, $5 \mathrm{H}$ ), 4.40 (ddt, ${ }^{3} \mathrm{JH}_{\mathrm{H}-\mathrm{F}}=25.6 \mathrm{~Hz},{ }^{3} \mathrm{JH}_{\mathrm{H}-\mathrm{F}}=4.7 \mathrm{~Hz},{ }^{3} \mathrm{JH}-\mathrm{H}=4.7 \mathrm{~Hz}, 1 \mathrm{H}$ ), 3.24 (dd, $\left.{ }^{1} J_{H-H}=19.3 \mathrm{~Hz},{ }^{3} J_{H-H}=4.4 \mathrm{~Hz}, 1 \mathrm{H}\right), 3.09\left(\mathrm{dd},{ }^{1}{ }^{H} \mathrm{H}-\mathrm{H}=19.2 \mathrm{~Hz},{ }^{3} \mathrm{~J}_{\mathrm{H}-\mathrm{H}}=\right.$ $5.2 \mathrm{~Hz}, 1 \mathrm{H}), 2.99-2.70(\mathrm{~m}, 2 \mathrm{H}), 2.21-1.96(\mathrm{~m}, 2 \mathrm{H}), 1.78(\mathrm{~s}, 3 \mathrm{H}), 1.50$ (s, 3H). ${ }^{13} \mathrm{C}$ NMR $\left(75 \mathrm{MHz}, \mathrm{CDCl}_{3}\right) \delta 195.6,140.2,135.9,134.0,129.0$, 128.6, 128.4, 128.3, 126.3, $125.3\left(\mathrm{t},{ }^{1} J_{C-F}=247.8 \mathrm{~Hz}\right), 89.2\left(\mathrm{~d},{ }^{3} J_{C-F}=1.8\right.$ $\mathrm{Hz}), 45.0\left(\mathrm{t},{ }^{2} J_{C-F}=21.6 \mathrm{~Hz}\right), 38.6\left(\mathrm{t},{ }^{2} J_{C-F}=24.9 \mathrm{~Hz}\right), 35.8\left(\mathrm{t},{ }^{3} J_{C-F}=4.5\right.$ $\mathrm{Hz}), 28.0\left(\mathrm{dd},{ }^{3} J_{C-F}=6.3 \mathrm{~Hz},{ }^{3} J_{C-F}=4.4 \mathrm{~Hz}\right), 27.2\left(\mathrm{~d},{ }^{4} J_{C-F}=1.5 \mathrm{~Hz}\right), 22.5$ $\left(\mathrm{t},{ }^{4} \mathrm{~J}_{\mathrm{C}-\mathrm{F}}=3.0 \mathrm{~Hz}\right) .{ }^{19} \mathrm{~F}\{\mathrm{H}\} \mathrm{NMR}\left(282 \mathrm{MHz}, \mathrm{CDCl}_{3}\right) \delta-100.15$ (AB system, $\left.{ }^{2} J_{F-F}=247.3 \mathrm{~Hz}\right),-102.16\left(\right.$ AB system, $\left.{ }^{2} J_{F-F}=247.3 \mathrm{~Hz}\right)$. HRMS (ESI): calcd. for $\left[\mathrm{M}+\mathrm{Na}^{+}\left(\mathrm{C}_{21} \mathrm{H}_{23} \mathrm{NO}_{3} \mathrm{~F}_{2} \mathrm{Na}\right)=398.1538\right.$; found: 398.1534 (1 ppm).

4,4-difluoro-1-(4-fluorophenyl)-3-(2-nitropropan-2-yl)-6-phenylhexan1-one $5 b$

Pale yellow solid, yield $134 \mathrm{mg}(69 \%)$ from $150 \mathrm{mg}(0.49 \mathrm{mmol})$ of $3 \mathbf{b}$. $\mathrm{R}_{\mathrm{f}}$ $=0.4$ (hexane $/$ Ethyl Acetate: $9 / 1) \cdot \mathrm{mp}=89^{\circ} \mathrm{C} .{ }^{1} \mathrm{H} \mathrm{NMR}\left(300 \mathrm{MHz}, \mathrm{CDCl}_{3}\right)$ $\delta 8.10-7.93(\mathrm{~m}, 2 \mathrm{H}), 7.34-7.07(\mathrm{~m}, 7 \mathrm{H}), 4.35\left(\mathrm{ddt},{ }^{3} \mathrm{JH}_{\mathrm{F}}=25.3 \mathrm{~Hz},{ }^{3} \mathrm{~J}\right.$ $\left.F=4.8 \mathrm{~Hz},{ }^{3} J_{H-H}=4.8,1 \mathrm{H}\right), 3.20\left(\mathrm{dd},{ }^{1} J_{H-H}=19.2 \mathrm{~Hz},{ }^{3} J_{H-H}=4.4 \mathrm{~Hz}, 1 \mathrm{H}\right)$, $3.03\left(\mathrm{dd},{ }^{1} \mathrm{~J}_{\mathrm{H}-\mathrm{H}}=19.2 \mathrm{~Hz},{ }^{1} \mathrm{~J}_{\mathrm{H}-\mathrm{H}}=5.1 \mathrm{~Hz}, 1 \mathrm{H}\right), 2.95-2.68(\mathrm{~m}, 2 \mathrm{H}), 2.17-$ $1.93(\mathrm{~m}, 2 \mathrm{H}), 1.76(\mathrm{~s}, 3 \mathrm{H}), 1.50(\mathrm{~s}, 3 \mathrm{H}) .{ }^{13} \mathrm{C} \mathrm{NMR}\left(75 \mathrm{MHz}, \mathrm{CDCl}_{3}\right) \delta 194.0$, $166.3\left(\mathrm{~d},{ }^{1} J_{C-F}=256.3 \mathrm{~Hz}\right), 140.2,132.4\left(\mathrm{~d},{ }^{4} J_{C-F}=3.0 \mathrm{~Hz}\right), 131.0\left(\mathrm{~d},{ }^{3} J_{C-F}\right.$ $=9.5 \mathrm{~Hz}), 128.7,128.4,126.4,125.2\left(\mathrm{t},{ }^{1} J_{C-F}=247.8 \mathrm{~Hz}\right), 116.2\left(\mathrm{~d},{ }^{2} J_{C-F}\right.$ $=22.0 \mathrm{~Hz}), 89.2\left(\mathrm{~d},{ }^{3} J_{C-F}=1.5 \mathrm{~Hz}\right), 45.1\left(\mathrm{t},{ }^{2} J_{C-F}=21.7 \mathrm{~Hz}\right), 38.6\left(\mathrm{t},{ }^{2} J_{C-F}\right.$ $=24.9 \mathrm{~Hz}), 35.7\left(\mathrm{t},{ }^{3} J_{C-F}=4.5 \mathrm{~Hz}\right), 28.0\left(\mathrm{dd},{ }^{3} J_{C-F}=6.1 \mathrm{~Hz},{ }^{3} J_{C-F}=4.6 \mathrm{~Hz}\right)$, 27.0, 23.2, 22.7. ${ }^{19} \mathrm{~F}\{\mathrm{H}\} \mathrm{NMR}\left(282 \mathrm{MHz}, \mathrm{CDCl}_{3}\right) \delta-100.05$ (AB system, $\left.{ }^{2} J_{F-F}=247.5 \mathrm{~Hz}\right),-102.15\left(\mathrm{AB}\right.$ system, $\left.{ }^{2} J_{F-F}=247.5 \mathrm{~Hz}\right),-103.55(\mathrm{~s})$. HRMS (ESI): calcd. for $[\mathrm{M}+\mathrm{Na}]^{+}\left(\mathrm{C}_{21} \mathrm{H}_{22} \mathrm{NO}_{3} \mathrm{~F}_{3} \mathrm{Na}\right)=416.1444$; found: 416.1443 (0 ppm).

\section{1-(4-chlorophenyl)-4,4-difluoro-3-(2-nitropropan-2-yl)-6- phenylhexan-1-one $5 \mathrm{c}$}

Yellow oil, yield $140 \mathrm{mg}(73 \%)$ from $150 \mathrm{mg}(0.47 \mathrm{mmol})$ of $3 \mathrm{c}$. $\mathrm{R}_{\mathrm{f}}=0.6$ (hexane / Ethyl Acetate: 8/2). ${ }^{1} \mathrm{H} \mathrm{NMR}\left(300 \mathrm{MHz}, \mathrm{CDCl}_{3}\right) \delta 8.05-7.95$ (m, $2 \mathrm{H}), 7.58-7.51(\mathrm{~m}, 5 \mathrm{H}), 7.38-7.15(\mathrm{~m}, 2 \mathrm{H}), 4.41\left(\mathrm{ddt},{ }^{3} \mathrm{~J}_{\mathrm{H}-\mathrm{F}}=25.3 \mathrm{~Hz}\right.$, $\left.{ }^{3} \mathrm{JH}_{\mathrm{H}-\mathrm{F}}=4.8 \mathrm{~Hz},{ }^{3} \mathrm{JH}_{\mathrm{H}}=4.8 \mathrm{~Hz}, 1 \mathrm{H}\right), 3.27\left(\mathrm{dd},{ }^{2} \mathrm{JH}_{\mathrm{H}-\mathrm{H}}=19.3 \mathrm{~Hz},{ }^{3} \mathrm{~J}_{\mathrm{H}-\mathrm{H}}=4.4 \mathrm{~Hz}\right.$, $1 \mathrm{H}), 3.09\left(\mathrm{dd},{ }^{2} \mathrm{~J}_{\mathrm{H}-\mathrm{H}}=19.3 \mathrm{~Hz},{ }^{3} \mathrm{~J}_{\mathrm{H}-\mathrm{H}}=5.1 \mathrm{~Hz}, 1 \mathrm{H}\right), 3.02-2.74(\mathrm{~m}, 2 \mathrm{H})$,
$2.24-2.01$ (m, 2H), 1.83 (s, 3H), 1.56 (s, 3H). ${ }^{13} \mathrm{C} \mathrm{NMR} \mathrm{(75} \mathrm{MHz,} \mathrm{CDCl} 3$ ) $\delta$ 194.5, 140.6, 140.2, 134.2, 129.7, 129.4, 128.7, 128.4, 126.4, $125.2(\mathrm{t}$ $\left.{ }^{1} J_{C-F}=248.2 \mathrm{~Hz}\right), 89.2\left(\mathrm{~d},{ }^{3} J_{C-F}=1.6 \mathrm{~Hz}\right), 45.1\left(\mathrm{t},{ }^{2} J_{C-F}=21.6 \mathrm{~Hz}\right), 38.6(\mathrm{t}$, $\left.{ }^{2} J_{C-F}=24.9 \mathrm{~Hz}\right), 35.8\left(\mathrm{t},{ }^{3} J_{C-F}=4.5 \mathrm{~Hz}\right), 28.0\left(\mathrm{dd},{ }^{3} J_{C-F}=6.2 \mathrm{~Hz},{ }^{3} J_{C-F}=4.5\right.$ $\mathrm{Hz})$, 23.9, $22.8\left(\mathrm{t},{ }^{4} \mathrm{~J}_{\mathrm{C}-\mathrm{F}}=3.0 \mathrm{~Hz}\right) .{ }^{19} \mathrm{~F}\{\mathrm{H}\} \mathrm{NMR}\left(282 \mathrm{MHz}, \mathrm{CDCl}_{3}\right) \delta-100.0$ (AB system, ${ }^{2} J_{F-F}=247.7 \mathrm{~Hz}$ ), $-102.2\left(\right.$ AB system, $\left.{ }^{2} \mathrm{JF}_{F-F}=247.7 \mathrm{~Hz}\right)$. HRMS (ESI): calcd. for $[\mathrm{M}+\mathrm{Na}]^{+}\left(\mathrm{C}_{21} \mathrm{H}_{22} \mathrm{NO}_{3} \mathrm{~F}_{2}{ }^{35} \mathrm{CINa}\right)=432.1149$; found: 432.1150 (0 ppm).

4,4-difluoro-1-(furan-2-yl)-3-(2-nitropropan-2-yl)-6-phenylhexan-1one $5 d$

Yellow oil, yield $117 \mathrm{mg}(72 \%)$ from $150 \mathrm{mg}(0.44 \mathrm{mmol})$ of $\mathbf{3 d}$. $R_{\mathrm{f}}=0.4$ (hexane / Ethyl Acetate: 8/2). ${ }^{1} \mathrm{H} \mathrm{NMR}\left(300 \mathrm{MHz}, \mathrm{CDCl}_{3}\right) \delta 7.69$ (dd, ${ }^{3} \mathrm{~J}_{\mathrm{H}-\mathrm{H}}$ $\left.=1.7 \mathrm{~Hz},{ }^{4} \mathrm{~J}_{\mathrm{H}-\mathrm{H}}=0.7 \mathrm{~Hz}, 1 \mathrm{H}\right), 7.39-7.18(\mathrm{~m}, 6 \mathrm{H}), 6.66\left(\mathrm{dd},{ }^{3} \mathrm{H}-\mathrm{H}=3.6 \mathrm{~Hz}\right.$, ${ }^{3} \mathrm{JH}_{\mathrm{H}-\mathrm{H}}=1.7 \mathrm{~Hz}, 1 \mathrm{H}$ ), 4.33 (ddt, ${ }^{3} \mathrm{~J}_{\mathrm{H}-\mathrm{F}}=25.2 \mathrm{~Hz},{ }^{3} \mathrm{~J}_{\mathrm{H}-\mathrm{F}}=4.8 \mathrm{~Hz},{ }^{3} \mathrm{~J}_{\mathrm{H}-\mathrm{H}}=4.8$ $\mathrm{Hz}, 1 \mathrm{H}), 3.16\left(\mathrm{dd},{ }^{2} \mathrm{~J}_{\mathrm{H}-\mathrm{H}}=19.2 \mathrm{~Hz},{ }^{3} \mathrm{~J}_{\mathrm{H}-\mathrm{H}}=4.5 \mathrm{~Hz}, 1 \mathrm{H}\right), 3.05\left(\mathrm{dd},{ }^{2}{ }^{\mathrm{H}-H}=\right.$ $\left.19.2 \mathrm{~Hz},{ }^{3} \mathrm{H}-\mathrm{H}=5.2 \mathrm{~Hz}, 1 \mathrm{H}\right), 3.00-2.75(\mathrm{~m}, 2 \mathrm{H}), 2.25-2.06(\mathrm{~m}, 2 \mathrm{H})$, 1.83 (s, 3H), 1.58 (s, 3H). ${ }^{13} \mathrm{C} \mathrm{NMR}\left(75 \mathrm{MHz}, \mathrm{CDCl}_{3}\right) \delta$ 185.0, 152.0, 147.0, 140.3, 128.7, 128.5, 126.4, $125.2\left(\mathrm{t},{ }^{1} J_{C-F}=245.3 \mathrm{~Hz}\right), 118.0,112.9,89.2$ $\left(\mathrm{d},{ }^{3} J_{C-F}=1.5 \mathrm{~Hz}\right), 44.7\left(\mathrm{t},{ }^{2} J_{C-F}=21.8 \mathrm{~Hz}\right), 38.6\left(\mathrm{t},{ }^{2} J_{C-F}=24.9 \mathrm{~Hz}\right), 35.6$ (t, $\left.{ }^{3} J_{C-F}=4.6 \mathrm{~Hz}\right), 28.0\left(\mathrm{dd},{ }^{3} J_{C-F}=6.1 \mathrm{~Hz},{ }^{3} J_{C-F}=4.6 \mathrm{~Hz}\right), 27.3\left(\mathrm{~d},{ }^{4} J_{C-F}=\right.$ $1.2 \mathrm{~Hz}), 22.4\left(\mathrm{dd},{ }^{4} J_{C-F}=2.7,{ }^{3} J_{C-F}=0.6 \mathrm{~Hz}\right) .{ }^{19} \mathrm{~F}\{\mathrm{H}\} \mathrm{NMR}(282 \mathrm{MHz}$, $\left.\mathrm{CDCl}_{3}\right) \delta-100.45$ (AB system, ${ }^{2} J_{F-F}=247.3 \mathrm{~Hz}$ ), -102.24 (AB system, ${ }^{2} J_{F-}$ $F=247.3 \mathrm{~Hz}$ ). HRMS (ESI): calcd. for $[\mathrm{M}+\mathrm{Na}]^{+}\left(\mathrm{C}_{19} \mathrm{H}_{21} \mathrm{NO}_{4} \mathrm{~F}_{2} \mathrm{Na}\right)=$ 388.1331 ; found 388.1333 (0 ppm).

\section{4,4-difluoro-1-(4-methoxyphenyl)-3-(2-nitropropan-2-yl)-6- phenylhexan-1-one $5 e$}

Pale yellow solid, yield $115 \mathrm{mg}(60 \%)$ from $150 \mathrm{mg}(0.47 \mathrm{mmol})$ of $3 \mathrm{e}$. Rf $=0.5$ (Petroleum Ether $/$ Ethyl Acetate: 8/2). $\mathrm{mp}=111^{\circ} \mathrm{C} .{ }^{1} \mathrm{H}$ NMR $(300$ $\left.\mathrm{MHz}, \mathrm{CDCl}_{3}\right) \delta 8.03-7.92(\mathrm{~m}, 2 \mathrm{H}), 7.31-7.17(\mathrm{~m}, 2 \mathrm{H}), 7.23-7.09(\mathrm{~m}$, 3H), $7.02-6.92(\mathrm{~m}, 2 \mathrm{H}), 4.38$ (ddt, ${ }^{3} \mathrm{~J}_{\mathrm{H}-\mathrm{F}}=25.7 \mathrm{~Hz},{ }^{3} \mathrm{~J}_{\mathrm{H}-\mathrm{F}}=4.7 \mathrm{~Hz},{ }^{3} \mathrm{~J}_{\mathrm{H}-\mathrm{H}}=$ $4.7 \mathrm{~Hz}, 1 \mathrm{H}), 3.89(\mathrm{~s}, 3 \mathrm{H}), 3.16\left(\mathrm{dd},{ }^{2} J_{H-H}=19.0 \mathrm{~Hz},{ }^{3} J_{H-H}=4.4 \mathrm{~Hz}, 1 \mathrm{H}\right)$, $3.01\left(\mathrm{dd},{ }^{2} \mathrm{~J}_{\mathrm{H}-\mathrm{H}}=19.1 \mathrm{~Hz},{ }^{3} \mathrm{~J}_{\mathrm{H}-\mathrm{H}}=5.2 \mathrm{~Hz}, 1 \mathrm{H}\right), 2.98-2.67(\mathrm{~m}, 2 \mathrm{H}), 2.20-$ $1.94(\mathrm{~m}, 2 \mathrm{H}), 1.76(\mathrm{~s}, 3 \mathrm{H}), 1.49(\mathrm{~s}, 3 \mathrm{H}) .{ }^{13} \mathrm{C} \mathrm{NMR}\left(75 \mathrm{MHz}, \mathrm{CDCl}_{3}\right) \delta 193.9$, 164.2, 140.3, 130.6, 129.0, 128.7, 128.5, 126.3, $125.4\left(\mathrm{t},{ }^{1} J_{C-F}=247.6 \mathrm{~Hz}\right)$, 114.2, $89.2\left(\mathrm{~d},{ }^{3} J_{C-F}=1.8 \mathrm{~Hz}\right), 55.7,45.0\left(\mathrm{t},{ }^{2} J_{C-F}=21.5 \mathrm{~Hz}\right), 38.6\left(\mathrm{t},{ }^{2} J_{C-F}\right.$ $=24.9 \mathrm{~Hz}), 35.4\left(\mathrm{dd},{ }^{3} J_{C-F}=5.0 \mathrm{~Hz},{ }^{3} J_{C-F}=3.8 \mathrm{~Hz}\right), 28.0\left(\mathrm{dd},{ }^{3} J_{C-F}=6.3\right.$ $\left.\mathrm{Hz},{ }^{3} J_{C-F}=4.4 \mathrm{~Hz}\right), 27.3\left(\mathrm{~d},{ }^{4} J_{C-F}=1.4 \mathrm{~Hz}\right), 22.5\left(\mathrm{t},{ }^{4} J_{C-F}=2.9 \mathrm{~Hz}\right) .{ }^{19} \mathrm{~F}\{\mathrm{H}\}$ $\operatorname{NMR}\left(471 \mathrm{MHz}, \mathrm{CDCl}_{3}\right) \delta-100.30\left(\mathrm{AB}\right.$ system, $\left.{ }^{2} \mathrm{~J} F-F=247.1 \mathrm{~Hz}\right),-101.94$ $\left(A B\right.$ system, ${ }^{2} J_{F-F}=247.1 \mathrm{~Hz}$ ). HRMS (ESI): calcd. for $[\mathrm{M}+\mathrm{Na}]^{+}$ $\left(\mathrm{C}_{22} \mathrm{H}_{25} \mathrm{NO}_{4} \mathrm{~F}_{2} \mathrm{Na}\right)=428.1644$; found: 428.1650 (1 ppm).

\section{General procedure 3 for the synthesis of difluoronitrones 6 and 7}

Representative procedure: 3-(1,1-difluoro-3-phenylpropyl)-5-phenyl3,4-dihydro-2H-pyrrole 1-oxide $6 \mathrm{a}$

To the difluoronitro-ketone $4 a\left(75 \mathrm{mg}, 0.22 \mathrm{mmol}\right.$ ) was added $\mathrm{NH}_{4} \mathrm{Cl}$ (35 $\mathrm{mg}, 0.65 \mathrm{mmol})$ in THF $(2 \mathrm{ml})$ and water $(1 \mathrm{ml})$ at $0{ }^{\circ} \mathrm{C}$. The reaction mixture was stirred for $5 \mathrm{mn}$ then $\mathrm{Zn}$ powder ( $212 \mathrm{mg}, 3.24 \mathrm{mmol}$ ) was added over $15 \mathrm{mn}$ and very slowly. The reaction was monitored using TLC each $5 \mathrm{mn}$. Prolonged reaction times will lead to the total reduction of the nitro groups into amines. At the end the reaction mixture was filtrated on celite, concentrated under vacuum, and purified by chromatography on silica gel, 6a $\left(44 \mathrm{mg}, 65 \%\right.$ ) was obtained as yellow solid. $R_{f}=0.3$ (hexane / Ethyl Acetate: $6 / 4) . \mathrm{mp}=114{ }^{\circ} \mathrm{C} .{ }^{1} \mathrm{H} \mathrm{NMR}\left(300 \mathrm{MHz}, \mathrm{CDCl}_{3}\right) \delta 8.37-8.18(\mathrm{~m}$, $2 \mathrm{H}), 7.49-7.39(\mathrm{~m}, 3 \mathrm{H}), 7.37-7.27(\mathrm{~m}, 2 \mathrm{H}), 7.27-7.13(\mathrm{~m}, 3 \mathrm{H}), 4.36$ (dd, $\left.{ }^{2} \mathrm{~J}_{\mathrm{H}-\mathrm{H}}=14.3 \mathrm{~Hz},{ }^{3} \mathrm{~J}_{\mathrm{H}-\mathrm{H}}=7.8 \mathrm{~Hz}, 1 \mathrm{H}\right), 4.25\left(\mathrm{dd},{ }^{2} \mathrm{~J}_{\mathrm{H}-\mathrm{H}}=14.3 \mathrm{~Hz},{ }^{3} \mathrm{~J}_{\mathrm{H}-\mathrm{H}}=\right.$ $9.7 \mathrm{~Hz}, 1 \mathrm{H}), 3.25\left(\mathrm{~d},{ }^{3} \mathrm{~J}_{\mathrm{H}-\mathrm{H}}=8.1 \mathrm{~Hz}, 2 \mathrm{H}\right), 3.12-2.93(\mathrm{~m}, 1 \mathrm{H}), 2.93-2.79$ $(\mathrm{m}, 2 \mathrm{H}), 2.32-2.09(\mathrm{~m}, 2 \mathrm{H}) .{ }^{13} \mathrm{C}$ NMR $\left(75 \mathrm{MHz}, \mathrm{CDCl}_{3}\right) \delta 139.9,139.4$, 130.7, 128.8, 128.7, 128.6, 128.3, 127.3, 126.6, $123.3\left(\mathrm{t},{ }^{1} \mathrm{JC-F}=244.0 \mathrm{~Hz}\right)$, $64.2\left(\mathrm{dd},{ }^{3} J_{C-F}=5.5 \mathrm{~Hz},{ }^{3} J_{C-F}=3.8 \mathrm{~Hz}\right), 37.0\left(\mathrm{t},{ }^{2} J_{C-F}=24.9 \mathrm{~Hz}\right), 36.5(\mathrm{t}$, $\left.{ }^{2} J_{C-F}=26.4 \mathrm{~Hz}\right), 31.4\left(\mathrm{dd},{ }^{3} J_{C-F}=5.6 \mathrm{~Hz},{ }^{3} J_{C-F}=4.3 \mathrm{~Hz}\right), 27.9\left(\mathrm{t},{ }^{3} J_{C-F}=\right.$ 
$4.7 \mathrm{~Hz}) .{ }^{19} \mathrm{~F}\{\mathrm{H}\} \mathrm{NMR}\left(471 \mathrm{MHz}, \mathrm{CDCl}_{3}\right) \delta-106.75(\mathrm{~d}, J=246.0 \mathrm{~Hz})$, $107.87(\mathrm{~d}, J=246.0 \mathrm{~Hz})$. HRMS (ESI): calcd. for $[\mathrm{M}+\mathrm{Na}]^{+}\left(\mathrm{C}_{19} \mathrm{H}_{19} \mathrm{NOF}_{2} \mathrm{Na}\right)$ $=338.1327$; found: 338.1330 (1 ppm).

\section{3-(1,1-difluoro-3-phenylpropyl)-5-(4-fluorophenyl)-3,4-dihydro-2H- pyrrole 1-oxide $6 \mathrm{~b}$}

Pale yellow solid, yield $42 \mathrm{mg}(61 \%)$ from $75 \mathrm{mg}(0.20 \mathrm{mmol})$ of $4 \mathbf{b} . \mathrm{R}_{\mathrm{f}}=$ 0.1 (hexane / Ethyl Acetate: 6/4). $\mathrm{mp}=155^{\circ} \mathrm{C} .{ }^{1} \mathrm{H} \mathrm{NMR}\left(300 \mathrm{MHz}, \mathrm{CDCl}_{3}\right)$ $\delta 8.42-8.27(\mathrm{~m}, 2 \mathrm{H}), 7.38-7.28(\mathrm{~m}, 2 \mathrm{H}), 7.28-7.07(\mathrm{~m}, 5 \mathrm{H}), 4.44-$ $4.18(\mathrm{~m}, 2 \mathrm{H}), 3.43-3.16(\mathrm{~m}, 2 \mathrm{H}), 3.16-2.94(\mathrm{~m}, 1 \mathrm{H}), 2.94-2.80(\mathrm{~m}, 2 \mathrm{H})$, $2.32-2.10(\mathrm{~m}, 2 \mathrm{H}) .{ }^{13} \mathrm{C}$ NMR $\left(75 \mathrm{MHz}, \mathrm{CDCl}_{3}\right) \delta 163.7\left(\mathrm{~d},{ }^{1} \mathrm{~J}_{\mathrm{C}-F}=252.8\right.$ $\mathrm{Hz}), 140.0,138.7,129.8\left(\mathrm{~d},{ }^{3} J_{C-F}=8.3 \mathrm{~Hz}\right), 128.9,128.4,126.7,125.1$, $123.3\left(\mathrm{t},{ }^{1} J_{C-F}=244.1 \mathrm{~Hz}\right), 115.8\left(\mathrm{~d},{ }^{2} J_{C-F}=21.6 \mathrm{~Hz}\right), 64.2,37.2\left(\mathrm{t},{ }^{2} J_{C-F}=\right.$ $24.9 \mathrm{~Hz}), 36.5\left(\mathrm{t},{ }^{2} J_{C-F}=26.4 \mathrm{~Hz}\right), 31.4,28.0\left(\mathrm{t},{ }^{3} J_{C-F}=4.6 \mathrm{~Hz}\right) .{ }^{19} \mathrm{~F}\{\mathrm{H}\}$ $\operatorname{NMR}\left(282 \mathrm{MHz}, \mathrm{CDCl}_{3}\right) \delta-106.86\left(\mathrm{AB}\right.$ system, $\left.{ }^{2} J_{F-F}=246.1 \mathrm{~Hz}\right),-107.32$ (s), -108.00 (AB system, $\left.{ }^{2} J_{F-F}=246.1 \mathrm{~Hz}\right)$. HRMS (ESI): calcd. for $[\mathrm{M}+\mathrm{Na}]^{+}$ $\left(\mathrm{C}_{19} \mathrm{H}_{18} \mathrm{NOF}_{3} \mathrm{Na}\right)=356.1233$; found: $356.1235(1 \mathrm{ppm})$.

\section{5-(4-chlorophenyl)-3-(1,1-difluoro-3-phenylpropyl)-3,4-dihydro-2H-} pyrrole 1-oxide $6 \mathrm{c}$

Pale white solid, yield $52 \mathrm{mg}(71 \%)$ from $75 \mathrm{mg}(0.20 \mathrm{mmol})$ of $4 \mathrm{c} . \mathrm{R}_{\mathrm{f}}=0.1$ (Petroleum Ether / Ethyl Acetate: 6/4). $\mathrm{mp}=159{ }^{\circ} \mathrm{C} .{ }^{1} \mathrm{H}$ NMR $(300 \mathrm{MHz}$, $\left.\mathrm{CDCl}_{3}\right) \delta 8.32-8.20(\mathrm{~m}, 2 \mathrm{H}), 7.45-7.37(\mathrm{~m}, 2 \mathrm{H}), 7.36-7.15(\mathrm{~m}, 5 \mathrm{H})$, $4.36\left(\mathrm{dd},{ }^{2} \mathrm{~J}_{\mathrm{H}-\mathrm{H}}=14.4 \mathrm{~Hz},{ }^{3} \mathrm{JH}_{\mathrm{H}-\mathrm{H}}=7.8 \mathrm{~Hz}, 1 \mathrm{H}\right), 4.24\left(\mathrm{dd},{ }^{2} \mathrm{~J}_{\mathrm{H}-\mathrm{H}}=14.4 \mathrm{~Hz}\right.$, $\left.{ }^{3} \mathrm{JH}_{\mathrm{H}-\mathrm{H}}=9.7 \mathrm{~Hz}, 1 \mathrm{H}\right), 3.24\left(\mathrm{~d},{ }^{3} \mathrm{JH}_{\mathrm{H}}=8.1 \mathrm{~Hz}, 2 \mathrm{H}\right), 3.15-2.92(\mathrm{~m}, 1 \mathrm{H}), 2.93$ $-2.83(\mathrm{~m}, 2 \mathrm{H}), 2.31-2.09(\mathrm{~m}, 2 \mathrm{H}) .{ }^{13} \mathrm{C} \mathrm{NMR}\left(75 \mathrm{MHz}, \mathrm{CDCl}_{3}\right) \delta 139.9$, 138.5, 136.4, 128.9, 128.9, 128.6, 128.4, 127.2, 126.7, 123.3 (t, ${ }^{1} J_{C-F}=$ $244.2 \mathrm{~Hz}), 64.3\left(\mathrm{t},{ }^{3} J_{C-F}=4.7 \mathrm{~Hz}\right), 37.1\left(\mathrm{t},{ }^{2} J_{C-F}=24.9 \mathrm{~Hz}\right), 36.5\left(\mathrm{t},{ }^{2} J_{C-F}=\right.$ $26.4 \mathrm{~Hz}), 31.3\left(\mathrm{t},{ }^{3} J_{C-F}=5.0 \mathrm{~Hz}\right), 28.0\left(\mathrm{t},{ }^{3} J_{C-F}=4.7 \mathrm{~Hz}\right) .{ }^{19} \mathrm{~F}$ NMR $(471$ $\left.\mathrm{MHz}, \mathrm{CDCl}_{3}\right) \delta-106.84\left(\mathrm{AB}\right.$ system, ${ }^{2} \mathrm{~J}_{F-F}=246.3 \mathrm{~Hz}$ ), -107.86 (AB system, $\left.{ }^{2} J_{F-F}=246.2 \mathrm{~Hz}\right)$. HRMS $(\mathrm{ESI})$ : calcd. for $[\mathrm{M}+\mathrm{Na}]^{+}\left(\mathrm{C}_{19} \mathrm{H}_{18} \mathrm{NOF}_{3} \mathrm{CINa}\right)=$ 372.0937; found: 372.0939 (0 ppm).

\section{3-(1,1-difluoro-3-phenylpropyl)-5-(furan-2-yl)-3,4-dihydro-2H-pyrrole} 1-oxide 6d

Yellow oil, yield $50 \mathrm{mg}(73 \%)$ from $75 \mathrm{mg}(0.22 \mathrm{mmol})$ of $4 \mathbf{d} . R_{f}=0.2$ (hexane / Ethyl Acetate: 2/8). ${ }^{1} \mathrm{H}$ NMR $\left(300 \mathrm{MHz}, \mathrm{CDCl}_{3}\right) \delta 7.79\left(\mathrm{~d},{ }^{3} \mathrm{~J}_{\mathrm{H}-\mathrm{H}}=\right.$ $3.2 \mathrm{~Hz}, 1 \mathrm{H}), 7.49\left(\mathrm{~d},{ }^{3} \mathrm{~J}_{\mathrm{H}-\mathrm{H}}=1.1 \mathrm{~Hz}, 1 \mathrm{H}\right), 7.39-7.13(\mathrm{~m}, 5 \mathrm{H}), 6.58\left(\mathrm{dd},{ }^{3} \mathrm{~J}_{\mathrm{H}}\right.$ $\left.H=3.4 \mathrm{~Hz},{ }^{4} \mathrm{~J}_{\mathrm{H}-\mathrm{H}}=1.8 \mathrm{~Hz}, 1 \mathrm{H}\right), 4.36-4.06(\mathrm{~m}, 2 \mathrm{H}), 3.38-3.14(\mathrm{~m}, 2 \mathrm{H})$, $3.15-2.92(\mathrm{~m}, 1 \mathrm{H}), 2.92-2.77(\mathrm{~m}, 2 \mathrm{H}), 2.33-2.07(\mathrm{~m}, 2 \mathrm{H}) .{ }^{13} \mathrm{C} N M R$ $\left(75 \mathrm{MHz}, \mathrm{CDCl}_{3}\right) \delta$ 144.9, 143.9, 140.0, 133.5, 128.8, 128.4, 126.6, 123.3 (t, $\left.{ }^{1} J_{C-F}=244.1 \mathrm{~Hz}\right), 115.0,112.6,62.3\left(\mathrm{dd},{ }^{3} J_{C-F}=5.3 \mathrm{~Hz},{ }^{3} J_{C-F}=4.0 \mathrm{~Hz}\right)$, $37.6\left(\mathrm{t},{ }^{2} J_{C-F}=26.4 \mathrm{~Hz}\right), 37.0\left(\mathrm{t},{ }^{2} J_{C-F}=24.9 \mathrm{~Hz}\right), 29.3\left(\mathrm{t},{ }^{3} J_{C-F}=5.0 \mathrm{~Hz}\right)$, $28.0\left(\mathrm{t},{ }^{3} \mathrm{JC}_{-} \mathrm{F}=4.7 \mathrm{~Hz}\right) \cdot{ }^{19} \mathrm{~F}\{\mathrm{H}\} \mathrm{NMR}\left(282 \mathrm{MHz}, \mathrm{CDCl}_{3}\right) \delta-106.02(\mathrm{AB}$ system, $\left.{ }^{2} J_{F-F}=246.1 \mathrm{~Hz}\right),-108.38\left(\right.$ AB system, $\left.{ }^{2} J_{F-F}=246.1 \mathrm{~Hz}\right)$. HRMS (ESI): calcd. for $[\mathrm{M}+\mathrm{Naa}]^{+}\left(\mathrm{C}_{17} \mathrm{H}_{17} \mathrm{NO}_{2} \mathrm{~F}_{2} \mathrm{Na}\right)=328.1120$; found: 328.1121 (0 ppm).

3-(1,1-difluoro-3-phenylpropyl)-5-(4-methoxyphenyl)-3,4-dihydro-2Hpyrrole 1-oxide 6e

Pale yellow solid, yield $55 \mathrm{mg}(80 \%)$ from $75 \mathrm{mg}(0.20 \mathrm{mmol})$ of $4 \mathrm{e} . \mathrm{R}_{\mathrm{f}}=$ 0.4 (Petroleum Ether / Ethyl Acetate: $6 / 4) . \mathrm{mp}=126^{\circ} \mathrm{C} .{ }^{1} \mathrm{H} \mathrm{NMR}(300 \mathrm{MHz}$, $\left.\mathrm{CDCl}_{3}\right) \delta 8.37-8.25(\mathrm{~m}, 2 \mathrm{H}), 7.38-7.25(\mathrm{~m}, 2 \mathrm{H}), 7.29-7.15(\mathrm{~m}, 3 \mathrm{H})$, $6.98-6.91(\mathrm{~m}, 2 \mathrm{H}), 4.39-4.15(\mathrm{~m}, 2 \mathrm{H}), 3.84(\mathrm{~s}, 3 \mathrm{H}), 3.26-3.19(\mathrm{~m}, 2 \mathrm{H})$, $3.12-2.93(\mathrm{~m}, 1 \mathrm{H}), 2.91-2.84(\mathrm{~m}, 2 \mathrm{H}), 2.30-2.07(\mathrm{~m}, 2 \mathrm{H}) .{ }^{13} \mathrm{C} N M R$ $\left(75 \mathrm{MHz}, \mathrm{CDCl}_{3}\right) \delta 161.3,140.0,139.3,129.3,128.8,128.4,126.6,123.4$ $\left(\mathrm{t},{ }^{1} J_{C-F}=245.2 \mathrm{~Hz}\right), 121.6,113.9,63.7\left(\mathrm{t},{ }^{3} J_{C-F}=4.7 \mathrm{~Hz}\right), 55.4,37.1(\mathrm{t}$, $\left.{ }^{2} J_{C-F}=24.9 \mathrm{~Hz}\right), 36.5\left(\mathrm{t},{ }^{2} J_{C-F}=26.4 \mathrm{~Hz}\right), 31.4\left(\mathrm{t},{ }^{3} J_{C-F}=5.0 \mathrm{~Hz}\right), 28.0(\mathrm{t}$, $\left.{ }^{3} J_{C-F}=4.7 \mathrm{~Hz}\right) .{ }^{19} \mathrm{~F}\{\mathrm{H}\} \mathrm{NMR}\left(376 \mathrm{MHz}, \mathrm{CDCl}_{3}\right) \delta-106.9$ (AB system, ${ }^{2} J_{F-F}$ $=246.0 \mathrm{~Hz}$ ), -107.7 (AB system, ${ }^{2} J_{F-F}=246.0 \mathrm{~Hz}$ ). HRMS (ESI): calcd. for $[\mathrm{M}+\mathrm{Na}]^{+}\left(\mathrm{C}_{20} \mathrm{H}_{21} \mathrm{NO}_{2} \mathrm{~F}_{2} \mathrm{Na}\right)=368.1433$; found: 368.1435 (1 ppm).

3-(1,1-difluoro-3-phenylpropyl)-2,2-dimethyl-5-phenyl-3,4-dihydro$2 \mathrm{H}$-pyrrole 1-oxide $7 \mathrm{a}$
White solid, yield $62 \mathrm{mg}(90 \%)$ from $75 \mathrm{mg}(0.20 \mathrm{mmol})$ of $5 \mathrm{a}$. $\mathrm{R}_{\mathrm{f}}=0.4$ (hexane / Ethyl Acetate: 6/4). $\mathrm{mp}=110{ }^{\circ} \mathrm{C} .{ }^{1} \mathrm{H} \mathrm{NMR}\left(300 \mathrm{MHz}, \mathrm{CDCl}_{3}\right) \delta$ $8.44-8.26(\mathrm{~m}, 2 \mathrm{H}), 7.53-7.40(\mathrm{~m}, 3 \mathrm{H}), 7.37-7.16(\mathrm{~m}, 5 \mathrm{H}), 3.23-3.00$ $(\mathrm{m}, 2 \mathrm{H}), 3.00-2.84(\mathrm{~m}, 2 \mathrm{H}), 2.85-2.59(\mathrm{~m}, 1 \mathrm{H}), 2.41-2.07(\mathrm{~m}, 2 \mathrm{H})$, $1.65(\mathrm{~s}, 3 \mathrm{H}), 1.53(\mathrm{~s}, 3 \mathrm{H}) .{ }^{13} \mathrm{C} \mathrm{NMR}\left(75 \mathrm{MHz}, \mathrm{CDCl}_{3}\right) \delta$ 140.2, 136.0, 130.5, 129.4, 128.9, 128.7, 128.5, 127.4, 126.7, $123.7\left(\mathrm{t},{ }^{1} J_{C-F}=245.2 \mathrm{~Hz}\right), 77.0$, $48.5\left(\mathrm{t},{ }^{2} J_{C-F}=24.1 \mathrm{~Hz}\right), 38.9\left(\mathrm{t},{ }^{2} J_{C-F}=25.1 \mathrm{~Hz}\right), 28.5\left(\mathrm{dd},{ }^{3} J_{C-F}=7.0 \mathrm{~Hz}\right.$, $\left.{ }^{3} J_{C-F}=4.0 \mathrm{~Hz}\right), 28.1\left(\mathrm{t},{ }^{3} \mathrm{JC}_{\mathrm{C}-\mathrm{F}}=5.0 \mathrm{~Hz}\right), 27.3\left(\mathrm{~d},{ }^{4} \mathrm{~J}_{C-F}=1.2 \mathrm{~Hz}\right), 21.0\left(\mathrm{t},{ }^{3} \mathrm{JC}_{\mathrm{C}}\right.$ $F=3.3 \mathrm{~Hz}) .{ }^{19} \mathrm{~F}\{\mathrm{H}\} \mathrm{NMR}\left(282 \mathrm{MHz}, \mathrm{CDCl}_{3}\right) \delta-102.75$ (AB system, ${ }^{2} \mathrm{~J} F-F=$ $247.0 \mathrm{~Hz}$ ), -104.91 (AB system, ${ }^{2} \mathrm{~J}_{F-F}=247.0 \mathrm{~Hz}$ ). HRMS (ESI): calcd. for $\left[\mathrm{M}+\mathrm{Na}^{+}\left(\mathrm{C}_{21} \mathrm{H}_{23} \mathrm{NOF}_{2} \mathrm{Na}\right)=366.1640\right.$; found: 366.1644 (1 ppm).

3-(1,1-difluoro-3-phenylpropyl)-5-(4-fluorophenyl)-2,2-dimethyl-3,4dihydro-2H-pyrrole 1-oxide $7 \mathrm{~b}$

White solid, yield $38 \mathrm{mg}(55 \%)$ from $75 \mathrm{mg}(0.19 \mathrm{mmol})$ of $\mathbf{5 b}$. $\mathrm{R}_{\mathrm{f}}=0.2$ (hexane / Ethyl Acetate: 6/4). $\mathrm{mp}=119{ }^{\circ} \mathrm{C} .{ }^{1} \mathrm{H}$ NMR $\left(300 \mathrm{MHz}, \mathrm{CDCl}_{3}\right) \delta$ $8.46-8.29(\mathrm{~m}, 2 \mathrm{H}), 7.39-7.27(\mathrm{~m}, 2 \mathrm{H}), 7.30-7.18(\mathrm{~m}, 3 \mathrm{H}), 7.19-7.04$ $(\mathrm{m}, 2 \mathrm{H}), 3.17-2.98(\mathrm{~m}, 2 \mathrm{H}), 2.99-2.83(\mathrm{~m}, 2 \mathrm{H}), 2.73(\mathrm{~m}, 1 \mathrm{H}), 2.38-$ $2.12(\mathrm{~m}, 2 \mathrm{H}), 1.64(\mathrm{~s}, 3 \mathrm{H}), 1.53(\mathrm{~s}, 3 \mathrm{H}) .{ }^{13} \mathrm{C} \mathrm{NMR}\left(75 \mathrm{MHz}, \mathrm{CDCl}_{3}\right) \delta 163.4$ $\left(\mathrm{d},{ }^{1} J_{C-F}=252.7 \mathrm{~Hz}\right), 140.2,135.1,129.7\left(\mathrm{~d},{ }^{3} J_{C-F}=8.3 \mathrm{~Hz}\right), 128.9,128.4$, 126.7, $125.8\left(\mathrm{~d},{ }^{4} J_{C-F}=3.4 \mathrm{~Hz}\right), 123.6\left(\mathrm{t},{ }^{1} J_{C-F}=245.1 \mathrm{~Hz}\right), 115.7\left(\mathrm{~d},{ }^{2} J_{C-F}\right.$ $=21.6 \mathrm{~Hz}), 76.9,48.4\left(\mathrm{t},{ }^{2} J_{C-F}=24.2 \mathrm{~Hz}\right), 38.9\left(\mathrm{t},{ }^{2} J_{C-F}=25.1 \mathrm{~Hz}\right), 28.5$ $\left(\mathrm{dd},{ }^{3} J_{C-F}=7.2,{ }^{3} J_{C-F}=4.0 \mathrm{~Hz}\right), 28.0\left(\mathrm{t},{ }^{3} J_{C-F}=5.0 \mathrm{~Hz}\right), 27.3,21.0\left(\mathrm{t},{ }^{4} J_{C-F}\right.$ $=3.6 \mathrm{~Hz}) \cdot{ }^{19} \mathrm{~F}\{\mathrm{H}\} \mathrm{NMR}\left(282 \mathrm{MHz}, \mathrm{CDCl}_{3}\right) \delta-102.61$ (AB system, ${ }^{2} J_{F-F}=$ $247.3 \mathrm{~Hz}$ ), -104.92 (AB system, ${ }^{2} J_{F-F}=247.3 \mathrm{~Hz}$ ), -108.05 (s). HRMS (ESI): calcd. for $[\mathrm{M}+\mathrm{Na}]^{+}\left(\mathrm{C}_{21} \mathrm{H}_{22} \mathrm{NOF}_{3} \mathrm{Na}\right)=384.1545$; found: $384.1543(1$ ppm).

\section{5-(4-chlorophenyl)-3-(1,1-difluoro-3-phenylpropyl)-2,2-dimethyl-3,4-} dihydro-2H-pyrrole 1-oxide 7c

Yellow oil, yield $37 \mathrm{mg}(54 \%)$ from $75 \mathrm{mg}(0.18 \mathrm{mmol})$ of $5 \mathrm{c} . \mathrm{R}_{\mathrm{f}}=0.2$ (hexane / Ethyl Acetate: 6/4). ${ }^{1} \mathrm{H} \mathrm{NMR}\left(300 \mathrm{MHz}, \mathrm{CDCl}_{3}\right) \delta 8.32-8.27$ (m, $2 \mathrm{H}), 7.43-7.39(\mathrm{~m}, 2 \mathrm{H}), 7.36-7.29(\mathrm{~m}, 2 \mathrm{H}), 7.28-7.20(\mathrm{~m}, 3 \mathrm{H}), 3.17-$ $3.00(\mathrm{~m}, 2 \mathrm{H}), 2.95-2.84(\mathrm{~m}, 2 \mathrm{H}), 2.82-2.64(\mathrm{~m}, 1 \mathrm{H}), 2.33-2.14(\mathrm{~m}, 2 \mathrm{H})$, $1.64(\mathrm{~s}, 3 \mathrm{H}), 1.52$ (s, 3H). ${ }^{13} \mathrm{C} \mathrm{NMR}\left(75 \mathrm{MHz}, \mathrm{CDCl}_{3}\right) \delta 140.1,136.0,135.4$, 128.9, 128.7, 128.4, 128.4, 127.8, 126.7, $123.6\left(\mathrm{t},{ }^{1} J_{F-F}=245.3 \mathrm{~Hz}\right), 78.8$, $48.4\left(\mathrm{t},{ }^{2} J_{F-F}=24.2 \mathrm{~Hz}\right), 38.9\left(\mathrm{t},{ }^{2} J_{F-F}=25.1 \mathrm{~Hz}\right), 28.4\left(\mathrm{dd},{ }^{3} J_{F-F}=7.1 \mathrm{~Hz}\right.$, $\left.{ }^{3} J_{F-F}=4.0 \mathrm{~Hz}\right), 28.0\left(\mathrm{dd},{ }^{3} J_{F-F}=5.0 \mathrm{~Hz},{ }^{3} J_{F-F}=0.1 \mathrm{~Hz}\right), 27.2\left(\mathrm{~d},{ }^{4} J_{F-F}=1.6\right.$ $\mathrm{Hz}), 21.0\left(\mathrm{dd},{ }^{3} J_{F-F}=4.2 \mathrm{~Hz},{ }^{3} J_{F-F}=3.1 \mathrm{~Hz}\right) .{ }^{19} \mathrm{~F}\{\mathrm{H}\} \mathrm{NMR}\left(282 \mathrm{MHz}, \mathrm{CDCl}_{3}\right)$ $\delta$-102.68 (AB system, ${ }^{2} J_{F-F}=247.3 \mathrm{~Hz}$ ), -104.95 (AB system, ${ }^{2} J_{F-F}=247.3$ $\mathrm{Hz})$. HRMS (ESI): calcd. for $[\mathrm{M}+\mathrm{Na}]^{+}\left(\mathrm{C}_{21} \mathrm{H}_{22} \mathrm{NOF}_{2}{ }^{35} \mathrm{CINa}\right)=400.1250$; found: 400.1255 (1 ppm).

\section{3-(1,1-difluoro-3-phenylpropyl)-5-(furan-2-yl)-2,2-dimethyl-3,4-} dihydro-2H-pyrrole 1-oxide 7d

Yellow oil, yield $48 \mathrm{mg}(70 \%)$ from $75 \mathrm{mg}(0.21 \mathrm{mmol})$ of $\mathbf{5 d} . \mathrm{R}_{\mathrm{f}}=0.3$ (hexane / Ethyl Acetate: $2 / 8) .{ }^{1} \mathrm{H} \mathrm{NMR}\left(300 \mathrm{MHz}, \mathrm{CDCl}_{3}\right) \delta 7.79\left(\mathrm{~d},{ }^{3} \mathrm{~J}_{\mathrm{H}-\mathrm{H}=}\right.$ $3.5 \mathrm{~Hz}, 1 \mathrm{H}), 7.48\left(\mathrm{dd},{ }^{3} \mathrm{~J}_{H-H}=1.8,{ }^{4} \mathrm{~J}_{H-H}=0.8 \mathrm{~Hz}, 1 \mathrm{H}\right), 7.36-7.28(\mathrm{~m}, 2 \mathrm{H})$, $7.27-7.18(\mathrm{~m}, 3 \mathrm{H}), 6.58\left(\mathrm{dd},{ }^{3} \mathrm{JH}_{\mathrm{H} H}=3.5 \mathrm{~Hz},{ }^{3} \mathrm{JH}_{\mathrm{H}}=1.8 \mathrm{~Hz}, 1 \mathrm{H}\right), 3.20-$ $2.98(\mathrm{~m}, 2 \mathrm{H}), 2.95-2.84(\mathrm{~m}, 2 \mathrm{H}), 2.84-2.61(\mathrm{~m}, 1 \mathrm{H}), 2.38-2.07(\mathrm{~m}, 2 \mathrm{H})$, $1.61(\mathrm{~s}, 3 \mathrm{H}), 1.51(\mathrm{~s}, 3 \mathrm{H}) .{ }^{13} \mathrm{C} \mathrm{NMR}\left(75 \mathrm{MHz}, \mathrm{CDCl}_{3}\right) \delta 145.4,143.5,140.1$, 130.0, 128.8, 128.4, 126.6, $123.6\left(\mathrm{dd},{ }^{1} J_{C-F}=246.0 \mathrm{~Hz},{ }^{1} J_{C-F}=244.3 \mathrm{~Hz}\right)$, 114.4, 112.5, 75.6, $49.2\left(\mathrm{t},{ }^{2} J_{C-F}=24.1 \mathrm{~Hz}\right), 38.8\left(\mathrm{t},{ }^{2} J_{C-F}=25.1 \mathrm{~Hz}\right), 28.0$ (dd, $\left.{ }^{3} J_{C-F}=5.7 \mathrm{~Hz},{ }^{3} J_{C-F}=4.3 \mathrm{~Hz}\right), 27.0\left(\mathrm{~d},{ }^{4} J_{C-F}=1.6 \mathrm{~Hz}\right), 26.5\left(\mathrm{dd},{ }^{3} J_{C-F}=\right.$ $\left.7.2 \mathrm{~Hz},{ }^{3} J_{C-F}=4.1 \mathrm{~Hz}\right), 20.9\left(\mathrm{dd},{ }^{4} \mathrm{~J}_{C-F}=4.6 \mathrm{~Hz},{ }^{4} J_{C-F}=2.9 \mathrm{~Hz}\right) .{ }^{19} \mathrm{~F}\{\mathrm{H}\} \mathrm{NMR}$ $\left(282 \mathrm{MHz}, \mathrm{CDCl}_{3}\right) \delta-103.27\left(\mathrm{AB}\right.$ system, $\left.{ }^{2} J_{F-F}=246.8 \mathrm{~Hz}\right),-104.94(\mathrm{AB}$ system, $\left.\quad{ }^{2} J_{F-F}=246.8 \mathrm{~Hz}\right)$. HRMS $(\mathrm{ESI}):$ calcd. for $[\mathrm{M}+\mathrm{Na}]^{+}$ $\left(\mathrm{C}_{19} \mathrm{H}_{21} \mathrm{NO}_{2} \mathrm{~F}_{2} \mathrm{Na}\right)=356.1433$; found: 356.1436 (1 ppm).

\section{3-(1,1-difluoro-3-phenylpropyl)-5-(4-methoxyphenyl)-2,2-dimethyl-} 3,4-dihydro-2H-pyrrole 1-oxide $7 \mathrm{e}$

White solid, yield $59 \mathrm{mg}(85 \%)$ from $75 \mathrm{mg}(0.18 \mathrm{mmol})$ of 5 e. $R_{\mathrm{f}}=0.3$ (Petroleum Ether / Ethyl Acetate: $7 / 3) . \mathrm{mp}=152{ }^{\circ} \mathrm{C} .{ }^{1} \mathrm{H}$ NMR $(300 \mathrm{MHz}$, $\left.\mathrm{CDCl}_{3}\right)$ ठ $8.39-8.26(\mathrm{~m}, 2 \mathrm{H}), 7.37-7.28(\mathrm{~m}, 2 \mathrm{H}), 7.27-7.15(\mathrm{~m}, 3 \mathrm{H})$, 
$6.98-6.88(\mathrm{~m}, 2 \mathrm{H}), 3.81(\mathrm{~s}, 3 \mathrm{H}), 3.07-2.97(\mathrm{~m}, 2 \mathrm{H}), 2.96-2.81(\mathrm{~m}, 2 \mathrm{H})$, $2.79-2.57(\mathrm{~m}, 1 \mathrm{H}), 2.35-2.10(\mathrm{~m}, 2 \mathrm{H}), 1.62(\mathrm{~s}, 3 \mathrm{H}), 1.50(\mathrm{~s}, 3 \mathrm{H}) .{ }^{13} \mathrm{C}$ $\mathrm{NMR}\left(75 \mathrm{MHz}, \mathrm{CDCl}_{3}\right) \delta 160.9,140.1,135.7,129.2,128.7,128.3,126.5$, $123.6\left(\mathrm{dd},{ }^{1} J_{C-F}=245.4 \mathrm{~Hz},{ }^{1} J_{C-F}=244.6 \mathrm{~Hz}\right), 122.1,113.8,76.2\left(\mathrm{~d},{ }^{3} J_{C-F}\right.$ $=1.1 \mathrm{~Hz}), 55.3,48.2\left(\mathrm{t},{ }^{2} J_{C-F}=24.1 \mathrm{~Hz}\right), 38.7\left(\mathrm{t},{ }^{2} J_{C-F}=25.1 \mathrm{~Hz}\right), 28.3(\mathrm{dd}$, $\left.{ }^{3} J_{C-F}=7.0 \mathrm{~Hz},{ }^{3} J_{C-F}=3.9 \mathrm{~Hz}\right), 27.9\left(\mathrm{dd},{ }^{4} J_{C-F}=5.5 \mathrm{~Hz},{ }^{4} J_{C-F}=4.4 \mathrm{~Hz}\right), 27.1$ $\left(\mathrm{d},{ }^{4} J_{C-F}=1.5 \mathrm{~Hz}\right), 20.8\left(\mathrm{dd},{ }^{3} J_{C-F}=4.3 \mathrm{~Hz},{ }^{3} J_{C-F}=2.9 \mathrm{~Hz}\right) .{ }^{19} \mathrm{~F}\{\mathrm{H}\} \mathrm{NMR}$ $\left(471 \mathrm{MHz}, \mathrm{CDCl}_{3}\right) \delta-102.74\left(\mathrm{AB}\right.$ system, $\left.{ }^{2} J_{F-F}=246.9 \mathrm{~Hz}\right),-104.68$ (AB system, $\left.{ }^{2} J_{F-F}=246.9 \mathrm{~Hz}\right)$. HRMS (ESI): calcd. for $[\mathrm{M}+\mathrm{Na}]^{+}$ $\left(\mathrm{C}_{22} \mathrm{H}_{25} \mathrm{NO}_{2} \mathrm{~F}_{2} \mathrm{Na}\right)=396.1745$; found: 396.1745 (0 ppm).

\section{Synthesis of 5-((4-methoxybenzyl)oxy)pent-1-yn-3-one 9}

To the propargylic alcohol $8(3 \mathrm{~g}, 13.62 \mathrm{mmol})$ in acetone $(20 \mathrm{~mL})$ was added dropwise under magnetic stirring at room temperature, a concentrated $(5.4 \mathrm{M})$ solution of Jones reagent until disappearance of the starting material (TLC analysis). After addition of isopropanol (5.0 equiv), the reaction mixture was filtered and the filtrate was extracted with ethyl acetate $(3 \times 40 \mathrm{~mL})$. The combined organic phases were dried over $\mathrm{Na}_{2} \mathrm{SO}_{4}$, filtered and concentrated under vacuum. After purification by chromatography on silica gel, 5-((4-methoxybenzyl)oxy)pent-1-yn-3-one 9 was obtained as colorless oil $(2.08 \mathrm{~g}, 70 \%)$. $R_{f}=0.6$ (Petroleum Ether Ethyl Acetate: 8/2). ${ }^{1} \mathrm{H}$ NMR $\left(300 \mathrm{MHz}, \mathrm{CDCl}_{3}\right) \delta 7.30-7.18(\mathrm{~m}, 2 \mathrm{H}), 6.94$ $-6.77(\mathrm{~m}, 2 \mathrm{H}), 4.45(\mathrm{~s}, 2 \mathrm{H}), 3.82-3.75(\mathrm{~m}, 5 \mathrm{H}), 3.23\left(\mathrm{~d},{ }^{5} \mathrm{~J}-\mathrm{H}=0.8 \mathrm{~Hz}\right.$, $1 \mathrm{H}), 2.85\left(\mathrm{td},{ }^{3} \mathrm{~J}_{\mathrm{H}-\mathrm{H}}=6.2 \mathrm{~Hz},{ }^{5} \mathrm{JH}_{\mathrm{H}}=0.8 \mathrm{~Hz}, 2 \mathrm{H}\right) .{ }^{13} \mathrm{C} \mathrm{NMR}\left(75 \mathrm{MHz}, \mathrm{CDCl}_{3}\right)$ ठ 185.3, 159.4, 130.0, 129.5, 113.9, 81.3, 79.1, 73.0, 64.3, 55.4, 45.7. HRMS (ESI): calcd. for $[\mathrm{M}+\mathrm{Na}]^{+}\left(\mathrm{C}_{13} \mathrm{H}_{14} \mathrm{O}_{3} \mathrm{Na}\right)=241.0835$; found: 241.0837 (1 ppm).

\section{Synthesis of 1-(((3,3-difluoropent-4-yn-1-yl)oxy)methyl)-4- methoxybenzene 10}

To the propargylic ketone $9(2 \mathrm{~g}, 9.16 \mathrm{mmol})$ one drop of $95 \%$ ethanol and DAST (2.42 $\mathrm{mL}, 18.32 \mathrm{mmol}, 2$ equiv) were added. The reaction mixture was stirred at $58^{\circ} \mathrm{C}$ for $8 \mathrm{~h}$. After coming back to room temperature and hydrolysis, the reaction mixture was extracted with $\mathrm{CH}_{2} \mathrm{Cl}_{2}(3 \times 40 \mathrm{~mL})$. The organic layers were separated, washed with water $(3 \times 20 \mathrm{~mL})$, dried over $\mathrm{MgSO}_{4}$ then filtrated on silica. After purification by chromatography on silica gel, the 1-(((3,3-difluoropent-4-yn-1-yl)oxy)methyl)-4methoxybenzene 10 was obtained as a yellow oil $(1.25 \mathrm{~g}, 57 \%)$. $R_{f}=0.4$ (Petroleum Ether/ Ethyl Acetate: 9/1). ${ }^{1} \mathrm{H}$ NMR $\left(300 \mathrm{MHz}, \mathrm{CDCl}_{3}\right) \delta 7.31$ $7.21(\mathrm{~m}, 3 \mathrm{H}), 6.95-6.81(\mathrm{~m}, 2 \mathrm{H}), 4.47(\mathrm{~s}, 2 \mathrm{H}), 3.81(\mathrm{~s}, 3 \mathrm{H}), 3.70\left(\mathrm{t},{ }^{3} \mathrm{JH}_{\mathrm{H}}\right.$ $=7.0 \mathrm{~Hz}, 2 \mathrm{H}), 2.78\left(\mathrm{t},{ }^{3} \mathrm{~J}_{\mathrm{H}-\mathrm{F}}=5.1 \mathrm{~Hz}, 1 \mathrm{H}\right), 2.41\left(\mathrm{tt},{ }^{3} \mathrm{~J}_{\mathrm{H}-\mathrm{F}}=14.7 \mathrm{~Hz},{ }^{3} \mathrm{~J}_{\mathrm{H}-\mathrm{H}}=\right.$ $6.9 \mathrm{~Hz}, 2 \mathrm{H}) .{ }^{13} \mathrm{C} \mathrm{NMR}\left(75 \mathrm{MHz}, \mathrm{CDCl}_{3}\right) \delta 159.4,130.1,129.5,114.0,113.0$ (t, $\left.{ }^{1} J_{C-F}=233.3 \mathrm{~Hz}\right), 76.4\left(\mathrm{t},{ }^{2} J_{C-F}=40.5 \mathrm{~Hz}\right), 75.7\left(\mathrm{t},{ }^{3} J_{C-F}=6.8 \mathrm{~Hz}\right), 73.0$, $63.9\left(\mathrm{t},{ }^{3} J_{C-F}=4.8 \mathrm{~Hz}\right), 55.4,39.4\left(\mathrm{t},{ }^{2}{ }^{\mathrm{C}-F}=25.7 \mathrm{~Hz}\right) .{ }^{19} \mathrm{~F}\{\mathrm{H}\} \mathrm{NMR}(282$ $\left.\mathrm{MHz}, \mathrm{CDCl}_{3}\right) \delta-82.75$ (s). HRMS (ESI): calcd. for $[\mathrm{M}+\mathrm{Na}]^{+}\left(\mathrm{C}_{13} \mathrm{H}_{14} \mathrm{O}_{2} \mathrm{~F}_{2} \mathrm{Na}\right)$ $=263.0854$; found: 263.0856 ( 1 ppm).

Synthesis of 4,4-difluoro-6-((4-methoxybenzyl)oxy)-1-phenylhex-2yn-1-ol 11a

The reaction was carried out with 1-(((3,3-difluoropent-4-yn-1yl)oxy)methyl)-4-methoxybenzene 10 ( $1 \mathrm{~g}, 4.16 \mathrm{mmol}$ ) with Benzaldehyde $(550 \mu \mathrm{L}, 5.41 \mathrm{mmol})$ according to the general procedure 1. After purification by chromatography on silica gel, 11a was obtained as yellow oil $(1.35 \mathrm{~g}, 95 \%)$. $\mathrm{R}_{\mathrm{f}}=0.4$ (Petroleum Ether/ Ethyl Acetate: $\left.7.5 / 2.5\right) .{ }^{1} \mathrm{H}$ NMR $\left(300 \mathrm{MHz}, \mathrm{CDCl}_{3}\right) \delta 7.55-7.34(\mathrm{~m}, 5 \mathrm{H}), 7.29-7.19(\mathrm{~m}, 2 \mathrm{H}), 6.94-$ $6.81(\mathrm{~m}, 2 \mathrm{H}), 5.48(\mathrm{bs}, 1 \mathrm{H}), 4.44(\mathrm{~s}, 2 \mathrm{H}), 3.79(\mathrm{~s}, 3 \mathrm{H}), 3.69\left(\mathrm{td},{ }^{3} \mathrm{~J}_{\mathrm{H}-\mathrm{H}}=6.8\right.$ $\left.\mathrm{Hz},{ }^{4} J_{H-F}=0.6 \mathrm{~Hz}, 2 \mathrm{H}\right), 2.57$ (bs, $\left.1 \mathrm{H}\right), 2.42\left(\mathrm{tt},{ }^{3} \mathrm{JH}_{\mathrm{F}}=14.0 \mathrm{~Hz},{ }^{3} \mathrm{~J}_{\mathrm{H}-\mathrm{H}}=6.8\right.$ $\mathrm{Hz}, 2 \mathrm{H}) .{ }^{13} \mathrm{C} \mathrm{NMR}\left(75 \mathrm{MHz}, \mathrm{CDCl}_{3}\right) \delta 159.4,139.1\left(\mathrm{t},{ }^{5} \mathrm{~J}_{\mathrm{C}-\mathrm{F}}=1.6 \mathrm{~Hz}\right), 130.0$, 129.5, 129.0, 128.9, 126.7, 114.0, 113.5 (t, $\left.{ }^{1} J_{C-F}=233.3 \mathrm{~Hz}\right), 87.2\left(\mathrm{t},{ }^{3} J_{C-F}\right.$ $=6.7 \mathrm{~Hz}), 79.2\left(\mathrm{t},{ }^{2} J_{C-F}=40.5 \mathrm{~Hz}\right), 72.9,64.2\left(\mathrm{t},{ }^{4} J_{C-F}=2.0 \mathrm{~Hz}\right), 63.9\left(\mathrm{t},{ }^{3} \mathrm{~J}_{C-}\right.$ $F=4.8 \mathrm{~Hz}), 55.4,39.5\left(\mathrm{t},{ }^{2} J_{C-F}=26.1 \mathrm{~Hz}\right) \cdot{ }^{19} \mathrm{~F}\{\mathrm{H}\} \mathrm{NMR}\left(282 \mathrm{MHz}, \mathrm{CDCl}_{3}\right)$ $\delta-81.73$ (s). HRMS (ESI): calcd. for $[\mathrm{M}+\mathrm{Na}]^{+}\left(\mathrm{C}_{20} \mathrm{H}_{20} \mathrm{O}_{3} \mathrm{~F}_{2} \mathrm{Na}\right)=369.1272$; found: $369.1272(0 \mathrm{ppm})$.
Synthesis of 4,4-difluoro-6-((4-methoxybenzyl)oxy)-3-(nitromethyl)-1phenylhexan-1-one 12a

The reaction was carried out with 4,4-difluoro-6-((4-methoxybenzyl)oxy)1-phenylhex-2-yn-1-ol 11a (1 g, $2.88 \mathrm{mmol})$ according to the general procedure 2. After purification by chromatography on silica gel, 12a was obtained as a colorless oil $(0.89 \mathrm{~g}, 76 \%)$. $\mathrm{R}_{\mathrm{f}}=0.3$ (Petroleum Ether/ Ethyl Acetate: $7 / 3)$. ${ }^{1} \mathrm{H}$ NMR $\left(300 \mathrm{MHz}, \mathrm{CDCl}_{3}\right) \delta 7.98-7.91(\mathrm{~m}, 2 \mathrm{H}), 7.65-$ $7.55(\mathrm{~m}, 1 \mathrm{H}), 7.52-7.43(\mathrm{~m}, 2 \mathrm{H}), 7.25-7.17(\mathrm{~m}, 2 \mathrm{H}), 6.87-6.80(\mathrm{~m}, 2 \mathrm{H})$, $4.73\left(\mathrm{dd},{ }^{2} \mathrm{~J}_{\mathrm{H}-\mathrm{H}}=13.4 \mathrm{~Hz},{ }^{3} \mathrm{JH}_{\mathrm{H}-\mathrm{H}}=5.9 \mathrm{~Hz}, 1 \mathrm{H}\right), 4.59\left(\mathrm{dd},{ }^{2} \mathrm{JH}_{\mathrm{H}}=13.4 \mathrm{~Hz},{ }^{3} \mathrm{~J}_{\mathrm{H}}\right.$ $H=5.0 \mathrm{~Hz}, 1 \mathrm{H}), 4.43(\mathrm{~s}, 2 \mathrm{H}), 3.78(\mathrm{~s}, 3 \mathrm{H}), 3.65\left(\mathrm{t},{ }^{3} \mathrm{~J}_{\mathrm{H}-\mathrm{H}}=5.9 \mathrm{~Hz}, 2 \mathrm{H}\right), 3.43$ (dd, $\left.{ }^{2} J_{H-H}=18.4 \mathrm{~Hz},{ }^{3} J_{H-H}=4.0 \mathrm{~Hz}, 1 \mathrm{H}\right), 3.24\left(\mathrm{dd},{ }^{2}{ }^{H}-H=18.4 \mathrm{~Hz},{ }^{3} \mathrm{~J}_{H-H}=\right.$ $8.9 \mathrm{~Hz}, 1 \mathrm{H}), 2.39-2.21(\mathrm{~m}, 2 \mathrm{H}) .{ }^{13} \mathrm{C} \mathrm{NMR}\left(75 \mathrm{MHz}, \mathrm{CDCl}_{3}\right) \delta 196.3,159.3$, $136.1,133.7,129.5,129.5,128.8,128.1,124.2\left(\mathrm{~d},{ }^{1} J_{C-F}=244.9 \mathrm{~Hz}\right), 113.9$, $73.5\left(\mathrm{t},{ }^{3} J_{C-F}=4.6 \mathrm{~Hz}\right), 73.2,63.4\left(\mathrm{t},{ }^{3} J_{C-F}=6.3 \mathrm{~Hz}\right), 55.3,39.3\left(\mathrm{t},{ }^{2} J_{C-F}=\right.$ $24.3 \mathrm{~Hz}), 35.3\left(\mathrm{t},{ }^{2} J_{C-F}=25.1 \mathrm{~Hz}\right), 34.9\left(\mathrm{t},{ }^{3} J_{C-F}=3.6 \mathrm{~Hz}\right) .{ }^{19} \mathrm{~F}\{\mathrm{H}\}$ NMR $(471$ $\mathrm{MHz}, \mathrm{CDCl}_{3}$ ) $\delta-99.53\left(\mathrm{AB}\right.$ system, ${ }^{2} \mathrm{~J}_{\mathrm{F}-\mathrm{F}}=253.2 \mathrm{~Hz}$ ), -100.47 (AB system, $\left.{ }^{2} J_{F-F}=253.2 \mathrm{~Hz}\right)$. HRMS $(E S I)$ : calcd. for $[\mathrm{M}+\mathrm{Na}]^{+}\left(\mathrm{C}_{21} \mathrm{H}_{23} \mathrm{NO}_{5} \mathrm{~F}_{2} \mathrm{Na}\right)=$ 430.1437; found: 430.1439 (1 ppm).

Synthesis of 3-(1,1-difluoro-3-((4-methoxybenzyl)oxy)propyl)-5phenyl-3,4-dihydro-2H-pyrrole 1-oxide 13a

The reaction was carried out with 4,4-difluoro-6-((4-methoxybenzyl)oxy)3-(nitromethyl)-1-phenylhexan-1-one 12a (75 mg, $0.18 \mathrm{mmol}$ ) according to the general procedure 3 . After purification by chromatography on silica gel, 13a was obtained as colorless oil $(53 \mathrm{mg}, 77 \%) . R_{f}=0.1$ (Petroleum Ether/ Ethyl Acetate: 6/4). ${ }^{1} \mathrm{H}$ NMR $\left(300 \mathrm{MHz}, \mathrm{CDCl}_{3}\right) \delta 8.36-8.21(\mathrm{~m}, 2 \mathrm{H}), 7.49$ $-7.40(\mathrm{~m}, 3 \mathrm{H}), 7.26-7.19(\mathrm{~m}, 2 \mathrm{H}), 6.95-6.81(\mathrm{~m}, 2 \mathrm{H}), 4.44(\mathrm{~s}, 2 \mathrm{H}), 4.41$ $-4.20(\mathrm{~m}, 2 \mathrm{H}), 3.79(\mathrm{~s}, 3 \mathrm{H}), 3.64\left(\mathrm{t},{ }^{3} \mathrm{~J}_{\mathrm{H}-\mathrm{H}}=5.9 \mathrm{~Hz}, 2 \mathrm{H}\right), 3.36-3.05(\mathrm{~m}$, $3 \mathrm{H}), 2.33-2.13(\mathrm{~m}, 2 \mathrm{H}) .{ }^{13} \mathrm{C} \mathrm{NMR}\left(75 \mathrm{MHz}, \mathrm{CDCl}_{3}\right) \delta 159.5,139.9,130.8$, 129.7, 129.5, 128.8, 128.6, 127.4, $123.5\left(\mathrm{t},{ }^{1} \mathrm{~J}_{C-F}=243.4 \mathrm{~Hz}\right), 114.1,73.2$, $64.4\left(\mathrm{dd},{ }^{3} J_{C-F}=6.1 \mathrm{~Hz},{ }^{3} J_{C-F}=3.7 \mathrm{~Hz}\right), 63.5\left(\mathrm{t},{ }^{3} J_{C-F}=6.3 \mathrm{~Hz}\right), 55.4,36.3$ $\left(\mathrm{t},{ }^{2} J_{C-F}=25.8 \mathrm{~Hz}\right), 35.8\left(\mathrm{t},{ }^{2} J_{C-F}=25.4 \mathrm{~Hz}\right), 31.5\left(\mathrm{dd},{ }^{3} J_{C-F}=6.2,{ }^{3} J_{C-F}=\right.$ $4.1 \mathrm{~Hz}) .{ }^{19} \mathrm{~F}\{\mathrm{H}\} \mathrm{NMR}\left(471 \mathrm{MHz}, \mathrm{CDCl}_{3}\right) \delta-103.73$ (AB system, ${ }^{2} \mathrm{~J} F-F=$ $249.2 \mathrm{~Hz}$ ), -104.83 (AB system, ${ }^{2} J_{F-F}=249.2 \mathrm{~Hz}$ ). HRMS (ESI): calcd. for $[\mathrm{M}+\mathrm{Na}]^{+}\left(\mathrm{C}_{21} \mathrm{H}_{23} \mathrm{NO}_{3} \mathrm{~F}_{2} \mathrm{Na}\right)=398.1538$; found: 398.1538 (0 ppm).

Synthesis of 4,4-difluoro-6-hydroxy-3-(nitromethyl)-1-phenylhexan1-one 14a

To protected nitro compound 12a ( $0.5 \mathrm{~g}, 1.22 \mathrm{mmol})$ in DCM $(20 \mathrm{~mL})$ and water $(1 \mathrm{~mL})$ was added under magnetic stirring at room temperature, DDQ (420 mg, $1.84 \mathrm{mmol})$ until disappearance of the starting material (TLC analysis). Then water was added and the reaction mixture was extracted with DCM $(3 \times 20 \mathrm{~mL})$. The combined organic phases were dried over $\mathrm{Na}_{2} \mathrm{SO}_{4}$, filtered and concentrated in vacuum. After purification by chromatography on silica gel, 4,4-difluoro-6-hydroxy-3-(nitromethyl)-1phenylhexan-1-one 14a was obtained as yellow oil $(345 \mathrm{mg}, 98 \%)$. $\mathrm{R}_{\mathrm{f}}=$ 0.4 (Petroleum Ether/ Ethyl Acetate: 6/4). ${ }^{1} \mathrm{H}$ NMR $\left(300 \mathrm{MHz}, \mathrm{CDCl}_{3}\right) \delta$ $7.99-7.91(\mathrm{~m}, 2 \mathrm{H}), 7.65-7.56(\mathrm{~m}, 1 \mathrm{H}), 7.53-7.43(\mathrm{~m}, 2 \mathrm{H}), 4.75$ (dd, $\left.{ }^{2} \mathrm{~J}_{\mathrm{H}-\mathrm{H}}=13.5 \mathrm{~Hz},{ }^{3} \mathrm{JH}_{\mathrm{H} H}=5.9 \mathrm{~Hz}, 1 \mathrm{H}\right), 4.55\left(\mathrm{dd},{ }^{2} \mathrm{~J}_{\mathrm{H}-\mathrm{H}}=13.5 \mathrm{~Hz},{ }^{3} \mathrm{JH}_{\mathrm{H} H}=5.5\right.$ $\mathrm{Hz}, 1 \mathrm{H}), 3.90\left(\mathrm{t},{ }^{3} \mathrm{JH}_{\mathrm{H}-\mathrm{H}}=5.9 \mathrm{~Hz}, 2 \mathrm{H}\right), 3.87-3.69(\mathrm{~m}, 1 \mathrm{H}), 3.45\left(\mathrm{dd},{ }^{2} \mathrm{~J}_{H-H}=\right.$ $\left.18.5 \mathrm{~Hz},{ }^{3} \mathrm{JH}_{\mathrm{H}-\mathrm{H}}=4.5 \mathrm{~Hz}, 1 \mathrm{H}\right), 3.25\left(\mathrm{dd},{ }^{2} \mathrm{~J}_{\mathrm{H}-\mathrm{H}}=18.5 \mathrm{~Hz},{ }^{3} \mathrm{~J}_{\mathrm{H}-\mathrm{H}}=8.2 \mathrm{~Hz}, 1 \mathrm{H}\right)$, $2.23\left(\mathrm{tt},{ }^{3} \mathrm{H}-\mathrm{F}=17.1 \mathrm{~Hz},{ }^{2} \mathrm{JH}_{\mathrm{H}-\mathrm{H}}=5.9 \mathrm{~Hz}, 2 \mathrm{H}\right), 2.08(\mathrm{bs}, 1 \mathrm{H}) .{ }^{13} \mathrm{C}$ NMR $(75$ $\mathrm{MHz}, \mathrm{CDCl}_{3} \delta 196.5,136.0,134.0,128.9,128.2,124.5(\mathrm{t}, J=245.1 \mathrm{~Hz})$, $73.7(\mathrm{t}, J=4.9 \mathrm{~Hz}), 56.5(\mathrm{t}, J=6.0 \mathrm{~Hz}), 39.5(\mathrm{t}, J=24.2 \mathrm{~Hz}), 37.3(\mathrm{t}, J=$ $24.1 \mathrm{~Hz}$ ), $35.2(\mathrm{dd}, J=4.2,3.3 \mathrm{~Hz}) .{ }^{19} \mathrm{~F}\{\mathrm{H}\} \mathrm{NMR}\left(471 \mathrm{MHz}, \mathrm{CDCl}_{3}\right) \delta-$ 100.56 (AB system, $\left.{ }^{2} J_{F-F}=252.5 \mathrm{~Hz}\right),-101.43\left(\right.$ AB system, ${ }^{2} J_{F-F}=252.5$ $\mathrm{Hz})$. HRMS (ESI): calcd. for $[\mathrm{M}+\mathrm{Naa}]^{+}\left(\mathrm{C}_{13} \mathrm{H}_{15} \mathrm{NO}_{4} \mathrm{~F}_{2} \mathrm{Na}\right)=310.0861$; found: 310.0863 (0 ppm).

Synthesis of 3-(1,1-difluoro-3-hydroxypropyl)-5-phenyl-3,4-dihydro2H-pyrrole 1-oxide 15a

The reaction was carried out with 4,4-difluoro-6-hydroxy-3-(nitromethyl)-1phenylhexan-1-one $14 \mathrm{a}(75 \mathrm{mg}, 0.26 \mathrm{mmol})$ according to the general 
procedure 3. After purification by chromatography on silica gel, 15a was obtained as white solid $\left(40 \mathrm{mg}, 60 \%\right.$ ). $R_{f}=0.1$ (Petroleum Ether/ Ethyl Acetate: $2.5 / 7.5) . \mathrm{mp}=147^{\circ} \mathrm{C} .{ }^{1} \mathrm{H}$ NMR $\left(300 \mathrm{MHz}, \mathrm{CD}_{3} \mathrm{OD}\right) \delta 8.37-8.27$ $(\mathrm{m}, 2 \mathrm{H}), 7.56-7.42(\mathrm{~m}, 3 \mathrm{H}), 4.46-4.18(\mathrm{~m}, 2 \mathrm{H}), 3.78\left(\mathrm{t},{ }^{3} \mathrm{~J}_{\mathrm{H}-\mathrm{H}}=6.3 \mathrm{~Hz}\right.$, $2 \mathrm{H}), 3.57-3.32(\mathrm{~m}, 3 \mathrm{H}), 2.31-2.14(\mathrm{~m}, 2 \mathrm{H}) .{ }^{13} \mathrm{C} \mathrm{NMR}\left(75 \mathrm{MHz}, \mathrm{CD}_{3} \mathrm{OD}\right)$ $\delta 144.8,132.5,129.6,129.5,129.2,125.3\left(\mathrm{t},{ }^{1} J_{C-F}=242.8 \mathrm{~Hz}\right), 65.0$ (dd, $\left.{ }^{3} J_{C-F}=6.1 \mathrm{~Hz},{ }^{3} J_{C-F}=4.0 \mathrm{~Hz}\right), 56.5\left(\mathrm{t},{ }^{3} J_{C-F}=6.2 \mathrm{~Hz}\right), 38.5\left(\mathrm{t},{ }^{2} J_{C-F}=24.2\right.$ $\mathrm{Hz}), 37.4\left(\mathrm{t},{ }^{2} \mathrm{JC}_{\mathrm{C}-\mathrm{F}}=25.8 \mathrm{~Hz}\right), 32.8\left(\mathrm{dd},{ }^{3} \mathrm{JC-F}=6.4 \mathrm{~Hz},{ }^{3} \mathrm{JC}_{\mathrm{F}}=4.1 \mathrm{~Hz}\right) .{ }^{19} \mathrm{~F}$ $\{\mathrm{H}\} \operatorname{NMR}\left(471 \mathrm{MHz}, \mathrm{CD}_{3} \mathrm{OD}\right) \delta-105.95\left(\mathrm{AB}\right.$ system, $\left.{ }^{2} J_{F-F}=248.4 \mathrm{~Hz}\right)$, 107.81 (AB system, ${ }^{2} J_{F-F}=248.4 \mathrm{~Hz}$ ). HRMS (ESI): calcd. for $[\mathrm{M}+\mathrm{Na}]^{+}$ $\left(\mathrm{C}_{13} \mathrm{H}_{15} \mathrm{NO}_{2} \mathrm{~F}_{2} \mathrm{Na}\right)=278.0963$; found: 278.0961 (1 ppm).

\section{3,3-difluoro-4-(nitromethyl)-6-oxo-6-phenylhexanoic acid 16a}

The reaction was carried out with 4,4-difluoro-6-hydroxy-3-(nitromethyl)-1phenylhexan-1-one 14a (200 mg, $0.70 \mathrm{mmol}$ ) based on the same procedure of the synthesis of compound 9. After purification by chromatography on silica gel, 16a was obtained as white solid (160 mg, $76 \%$ ). $R_{f}=0.2$ (Petroleum Ether/ Ethyl Acetate/methanol: 4.5/5/0.5). $\mathrm{mp}=$ $130{ }^{\circ} \mathrm{C} .{ }^{1} \mathrm{H}$ NMR $\left(300 \mathrm{MHz},\left(\mathrm{CD}_{3}\right)_{2} \mathrm{CO}\right) \delta 8.1-8.0(\mathrm{~m}, 2 \mathrm{H}), 7.7-7.6(\mathrm{~m}$, $1 \mathrm{H}), 7.6-7.5(\mathrm{~m}, 2 \mathrm{H}), 4.9\left(\mathrm{dd},{ }^{2} \mathrm{~J}-\mathrm{H}=14.2 \mathrm{~Hz},{ }^{3} \mathrm{JH}_{\mathrm{H}-\mathrm{H}}=6.8 \mathrm{~Hz}, 1 \mathrm{H}\right), 4.7$ (dd, $\left.{ }^{2} J_{H-H}=14.2 \mathrm{~Hz},{ }^{3} J_{H-H}=4.6 \mathrm{~Hz}, 1 \mathrm{H}\right), 4.2-4.0(\mathrm{~m}, 1 \mathrm{H}), 3.6$ (ddd, ${ }^{2} J_{H-H}=18.7$ $\left.\mathrm{Hz},{ }^{3} \mathrm{~J}_{\mathrm{H}-\mathrm{H}}=3.8 \mathrm{~Hz},{ }^{4} \mathrm{~J}_{\mathrm{H}-\mathrm{F}}=0.9 \mathrm{~Hz}, 1 \mathrm{H}\right), 3.5\left(\mathrm{dd},{ }^{2} \mathrm{~J}_{\mathrm{H}-\mathrm{H}}=18.7 \mathrm{~Hz},{ }^{3} \mathrm{~J}_{\mathrm{H}-\mathrm{H}}=9.2\right.$ $\mathrm{Hz}, 1 \mathrm{H}), 3.4-3.1(\mathrm{~m}, 2 \mathrm{H}) .{ }^{13} \mathrm{C}$ NMR $\left(75 \mathrm{MHz},\left(\mathrm{CD}_{3}\right)_{2} \mathrm{CO}\right) \delta 197.0,167.9$ $\left(\mathrm{dd},{ }^{3} J_{C-F}=8.9 \mathrm{~Hz},{ }^{3} J_{C-F}=4.6 \mathrm{~Hz}\right), 137.4,134.3,129.5,129.0,123.8\left(\mathrm{t},{ }^{1} J_{C}\right.$ $F=245.1 \mathrm{~Hz}), 74.2\left(\mathrm{t},{ }^{3} J_{C-F}=4.5 \mathrm{~Hz}\right), 40.2\left(\mathrm{t},{ }^{2} J_{C-F}=26.8 \mathrm{~Hz}\right), 39.7\left(\mathrm{t},{ }^{2} J_{C-F}\right.$ $=23.4 \mathrm{~Hz}), 36.1\left(\mathrm{dd},{ }^{3} J_{C-F}=4.9 \mathrm{~Hz},{ }^{3} J_{C-F}=2.6 \mathrm{~Hz}\right) .{ }^{19} \mathrm{~F}\{\mathrm{H}\} \mathrm{NMR}(471 \mathrm{MHz}$, $\left.\left(\mathrm{CD}_{3}\right)_{2} \mathrm{CO}\right) \delta-98.03\left(\mathrm{AB}\right.$ system, ${ }^{2} J_{F-F}=254.8 \mathrm{~Hz}$ ), -100.29 (AB system, $\left.{ }^{2} J_{F-F}=254.7 \mathrm{~Hz}\right)$. HRMS $(\mathrm{ESI})$ : calcd. for $[\mathrm{M}+\mathrm{Na}]^{+}\left(\mathrm{C}_{13} \mathrm{H}_{11} \mathrm{NO}_{5} \mathrm{~F}_{2} \mathrm{Na}\right)=$ 300.0689; found: 300.0684 (2 ppm).

\section{ethyl 3,3-difluoro-4-(nitromethyl)-6-oxo-6-phenylhexanoate 17a}

To 3,3-difluoro-4-(nitromethyl)-6-oxo-6-phenylhexanoic acid 16a (100 mg, $0.33 \mathrm{mmol}), \mathrm{N}, \mathrm{N}$-Dicyclohexylcarbodiimide "DCC" (137 mg, $0.66 \mathrm{mmol})$, and ethanol ( $39 \mu \mathrm{L}, 0.66 \mathrm{mmol}$ ), were added and mixed in $4 \mathrm{ml} \mathrm{DCM}$ for $24 \mathrm{~h}$. The reaction was monitored by TLC. At the end concentrated $\mathrm{NH}_{4} \mathrm{Cl}$ $(2 \mathrm{~mL})$ was added and the reaction mixture was extracted with DCM $(3 \times 10 \mathrm{~mL})$. The organic layers were separated, washed with water $(1 \times 10 \mathrm{~mL})$, dried over $\mathrm{Na}_{2} \mathrm{SO}_{4}$ and concentrated under vacuum. After purification by chromatography on silica gel, 17a (78 mg, 72\%) was obtained as pale yellow oil. $R_{f}=0.6$ (Petroleum Ether / Ethyl Acetate: 8/2). ${ }^{1} \mathrm{H}$ NMR $\left(300 \mathrm{MHz}, \mathrm{CDCl}_{3}\right) \delta 8.00-7.93(\mathrm{~m}, 2 \mathrm{H}), 7.66-7.57(\mathrm{~m}, 1 \mathrm{H})$, $7.53-7.45(\mathrm{~m}, 2 \mathrm{H}), 4.80\left(\mathrm{dd},{ }^{2} \mathrm{~J}_{\mathrm{H}-\mathrm{H}}=13.6 \mathrm{~Hz},{ }^{3} \mathrm{~J}_{\mathrm{H}-\mathrm{H}}=5.7 \mathrm{~Hz}, 1 \mathrm{H}\right), 4.62$ $\left(\mathrm{dd},{ }^{2} \mathrm{~J}_{\mathrm{H}-\mathrm{H}}=13.6 \mathrm{~Hz},{ }^{3} \mathrm{JH}_{\mathrm{H} H}=5.1 \mathrm{~Hz}, 1 \mathrm{H}\right), 4.21\left(\mathrm{q},{ }^{3} \mathrm{JH}_{\mathrm{H} H}=7.1 \mathrm{~Hz}, 2 \mathrm{H}\right), 4.02$ $-3.78(\mathrm{~m}, 1 \mathrm{H}), 3.45\left(\mathrm{dd},{ }^{2} \mathrm{~J}_{\mathrm{H}-\mathrm{H}}=18.4 \mathrm{~Hz},{ }^{3} \mathrm{~J}_{\mathrm{H}-\mathrm{H}}=4.7 \mathrm{~Hz}, 1 \mathrm{H}\right), 3.34(\mathrm{dd}$, $\left.{ }^{2} J_{H-H}=18.4 \mathrm{~Hz},{ }^{3} J_{H-H}=8.3 \mathrm{~Hz}, 1 \mathrm{H}\right), 3.11\left(\mathrm{dd},{ }^{2} \mathrm{JH}_{\mathrm{H}}=15.1 \mathrm{~Hz},{ }^{3} \mathrm{JH}_{\mathrm{H}-\mathrm{H}}=4.1\right.$ $\mathrm{Hz}, 1 \mathrm{H}), 3.05\left(\mathrm{dd},{ }^{2} \mathrm{~J}_{\mathrm{H}-\mathrm{H}}=15.1 \mathrm{~Hz},{ }^{3} \mathrm{JH}-\mathrm{H}=3.7 \mathrm{~Hz}, 1 \mathrm{H}\right), 1.29\left(\mathrm{t},{ }^{3} \mathrm{~J}_{\mathrm{H}-\mathrm{H}}=7.1\right.$ $\mathrm{Hz}, 3 \mathrm{H}) .{ }^{13} \mathrm{C}$ NMR $\left(75 \mathrm{MHz}, \mathrm{CDCl}_{3}\right) \delta 196.1,166.2\left(\mathrm{t},{ }^{3} \mathrm{JC}_{\mathrm{C}-\mathrm{F}}=7.4 \mathrm{~Hz}\right), 136.1$, 134.0, 129.0, 128.3, $121.8\left(\mathrm{t},{ }^{1} J_{C-F}=246.9 \mathrm{~Hz}\right), 73.6\left(\mathrm{t},{ }^{3} J_{C-F}=4.8 \mathrm{~Hz}\right)$, $61.9,40.7\left(\mathrm{t},{ }^{2} J_{C-F}=27.6 \mathrm{~Hz}\right), 39.1\left(\mathrm{t},{ }^{2} J_{C-F}=23.8 \mathrm{~Hz}\right), 34.8\left(\mathrm{t},{ }^{3} J_{C-F}=3.7\right.$ $\mathrm{Hz}$ ), 14.1. ${ }^{19} \mathrm{~F} \mathrm{NMR}\left(471 \mathrm{MHz}, \mathrm{CDCl}_{3}\right) \delta-97.24$ (AB system, ${ }^{2} J_{F-F}=257.7$ $\mathrm{Hz}$ ), -97.93 (AB system, ${ }^{2} \mathrm{~J}_{F-F}=257.7 \mathrm{~Hz}$ ). HRMS (ESI): calcd. for [M+Na] ${ }^{+}$ $\left(\mathrm{C}_{15} \mathrm{H}_{17} \mathrm{NO}_{5} \mathrm{~F}_{2} \mathrm{Na}\right)=352.0967$; found: 352.0972 (1 ppm).

\section{3-(3-ethoxy-1,1-difluoro-3-oxopropyl)-5-phenyl-3,4-dihydro-2H- pyrrole 1-oxide 18a}

The reaction was carried out with ethyl 3,3-difluoro-4-(nitromethyl)-6-oxo6-phenylhexanoate $17 \mathrm{a}(60 \mathrm{mg}, 0.18 \mathrm{mmol})$ according to the general procedure 3 . After purification by chromatography on silica gel, 18a was obtained as pale yellow oil $(42 \mathrm{mg}, 77 \%)$. $R_{f}=0.1$ (Petroleum Ether/ Ethyl Acetate: $5 / 5) .{ }^{1} \mathrm{H}$ NMR $\left(300 \mathrm{MHz}, \mathrm{CDCl}_{3}\right) \delta 8.36-8.22(\mathrm{~m}, 2 \mathrm{H}), 7.50-$ $7.36(\mathrm{~m}, 3 \mathrm{H}), 4.38-4.29(\mathrm{~m}, 2 \mathrm{H}), 4.20\left(\mathrm{q},{ }^{3} \mathrm{~J}_{\mathrm{H}-\mathrm{H}}=7.1 \mathrm{~Hz}, 2 \mathrm{H}\right), 3.61-3.15$ (m, 3H), $3.10-2.85(\mathrm{~m}, 2 \mathrm{H}), 1.28\left(\mathrm{t},{ }^{3} \mathrm{~J}_{\mathrm{H}-\mathrm{H}}=7.1 \mathrm{~Hz}, 3 \mathrm{H}\right) .{ }^{13} \mathrm{C} \mathrm{NMR}(75$ $\left.\mathrm{MHz}, \mathrm{CDCl}_{3}\right) \delta 166.3\left(\mathrm{t},{ }^{3} \mathrm{~J}_{\mathrm{C}-\mathrm{F}}=8.0 \mathrm{~Hz}\right), 139.3,130.8,128.7,128.6,127.3$, $121.1\left(\mathrm{t},{ }^{3} J_{C-F}=245.3 \mathrm{~Hz}\right), 64.0\left(\mathrm{dd},{ }^{3} J_{C-F}=5.7 \mathrm{~Hz},{ }^{3} J_{C-F}=3.5 \mathrm{~Hz}\right), 61.8$, $40.8\left(\mathrm{t},{ }^{2} J_{C-F}=28.2 \mathrm{~Hz}\right), 35.5\left(\mathrm{t},{ }^{2} J_{C-F}=25.1 \mathrm{~Hz}\right), 31.3\left(\mathrm{dd},{ }^{3} J_{C-F}=6.1 \mathrm{~Hz}\right.$, $\left.{ }^{3} J_{C-F}=4.1 \mathrm{~Hz}\right), 14.1 .{ }^{19} \mathrm{~F}\{\mathrm{H}\} \mathrm{NMR}\left(471 \mathrm{MHz}, \mathrm{CDCl}_{3}\right) \delta-101.23(\mathrm{~d}, \mathrm{AB}$ system, $\left.{ }^{2} J_{F-F}=253.8 \mathrm{~Hz}\right),-102.47\left(\mathrm{AB}\right.$ system, $\left.{ }^{2} J_{F-F}=253.8 \mathrm{~Hz}\right)$. HRMS (ESI): calcd. for $[\mathrm{M}+\mathrm{Na}]^{+}\left(\mathrm{C}_{15} \mathrm{H}_{17} \mathrm{NO}_{3} \mathrm{~F}_{2} \mathrm{Na}\right)=320.1069$; found: 320.1071 (1 ppm).

\section{3,3-difluoro-4-(nitromethyl)-6-oxo-6-phenylhexyl} methylbenzenesulfonate $19 \mathrm{a}$

To 4,4-difluoro-6-hydroxy-3-(nitromethyl)-1-phenylhexan-1-one 14a (150 $\mathrm{mg}, 0.52 \mathrm{mmol}$ ), $p$-toluenesulfonyl chloride (200 $\mathrm{mg}, 1.04 \mathrm{mmol}$ ), pyridine $(84 \mu \mathrm{L}, 1.04 \mathrm{mmol})$, and catalytic amount of DMAP were added and mixed in $5 \mathrm{ml} \mathrm{DCM}$ for $48 \mathrm{~h}$. The reaction was monitored by TLC. At the end saturated $\mathrm{NH}_{4} \mathrm{Cl}(2 \mathrm{~mL})$ was added and the reaction mixture was extracted with DCM $(3 \times 10 \mathrm{~mL})$. The organic layers were separated, washed with water (1×10 mL), dried over $\mathrm{Na}_{2} \mathrm{SO}_{4}$ and concentrated under vacuum. After purification by chromatography on silica gel, 19a (145 mg, 63\%) was obtained as white solid. $R_{f}=0.3$ (Petroleum Ether / Ethyl Acetate: 8/2). mp $=89^{\circ} \mathrm{C} .{ }^{1} \mathrm{H}$ NMR $\left(300 \mathrm{MHz}, \mathrm{CDCl}_{3}\right) \delta 7.99-7.89(\mathrm{~m}, 2 \mathrm{H}), 7.82-7.71(\mathrm{~m}$, $2 \mathrm{H}), 7.66-7.55(\mathrm{~m}, 1 \mathrm{H}), 7.54-7.42(\mathrm{~m}, 2 \mathrm{H}), 7.39-7.28(\mathrm{~m}, 2 \mathrm{H}), 4.67$ $\left(\mathrm{dd},{ }^{2} \mathrm{~J}_{\mathrm{H}-\mathrm{H}}=13.8 \mathrm{~Hz},{ }^{3} \mathrm{~J}_{\mathrm{H}-\mathrm{H}}=6.2 \mathrm{~Hz}, 1 \mathrm{H}\right), 4.48\left(\mathrm{dd},{ }^{2} \mathrm{~J}_{\mathrm{H}-\mathrm{H}}=13.8 \mathrm{~Hz},{ }^{3} \mathrm{~J}_{\mathrm{H}-\mathrm{H}}=\right.$ $5.2 \mathrm{~Hz}, 1 \mathrm{H}), 4.34-4.15(\mathrm{~m}, 2 \mathrm{H}), 3.74-3.51(\mathrm{~m}, 1 \mathrm{H}), 3.35\left(\mathrm{dd},{ }^{2}{ }^{2} \mathrm{H}-\mathrm{H}=18.6\right.$ $\left.\mathrm{Hz},{ }^{3} J_{H-H}=4.5 \mathrm{~Hz}, 1 \mathrm{H}\right), 3.21\left(\mathrm{dd},{ }^{2} J_{H-H}=18.6 \mathrm{~Hz},{ }^{3} \mathrm{~J}_{\mathrm{H}-\mathrm{H}}=8.1 \mathrm{~Hz}, 1 \mathrm{H}\right), 2.43$ (s, 3H), $2.42-2.25(\mathrm{~m}, 2 \mathrm{H}) .{ }^{13} \mathrm{C} \mathrm{NMR}\left(75 \mathrm{MHz}, \mathrm{CDCl}_{3}\right) \delta 195.8,145.4$, 135.9, 134.1, 132.4, 130.1, 128.9, 128.2, 128.0, $123.1\left(\mathrm{t},{ }^{1} J_{C-F}=246.0 \mathrm{~Hz}\right)$, $73.3\left(\mathrm{t},{ }^{3} J_{C-F}=4.8 \mathrm{~Hz}\right), 63.3\left(\mathrm{t},{ }^{3} J_{C-F}=5.9 \mathrm{~Hz}\right), 39.3\left(\mathrm{t},{ }^{2} J_{C-F}=23.7 \mathrm{~Hz}\right), 35.1$ $\left(\mathrm{dd},{ }^{3} J_{C-F}=4.3 \mathrm{~Hz},{ }^{3} J_{C-F}=3.0 \mathrm{~Hz}\right), 34.5\left(\mathrm{t},{ }^{2} J_{C-F}=24.8 \mathrm{~Hz}\right), 21.7 .{ }^{19} \mathrm{~F}\{\mathrm{H}\}$ $\operatorname{NMR}\left(471 \mathrm{MHz}, \mathrm{CDCl}_{3}\right) \delta-101.54$ (AB system, $\left.{ }^{2} J_{F-F}=253.2 \mathrm{~Hz}\right),-102.91$ (AB system, $\left.{ }^{2} J_{F-F}=253.2 \mathrm{~Hz}\right)$. HRMS (ESI): calcd. for $[\mathrm{M}+\mathrm{Na}]^{+}$ $\left(\mathrm{C}_{20} \mathrm{H}_{21} \mathrm{NO}_{6} \mathrm{~F}_{2} \mathrm{SNa}\right)=464.0950$; found: 464.0956 (1 ppm).

\section{3-(1,1-difluoro-3-(tosyloxy)propyl)-5-phenyl-3,4-dihydro-2H-pyrrole 1-oxide 20a}

The reaction was carried out with 3,3-difluoro-4-(nitromethyl)-6-oxo-6phenylhexyl 4-methylbenzenesulfonate $19 \mathrm{a}(100 \mathrm{mg}, 0.23 \mathrm{mmol})$ according to the general procedure 3 . After purification by chromatography on silica gel, 20a was obtained as colorless oil ( $75 \mathrm{mg}, 81 \%) . \mathrm{R}_{\mathrm{f}}=0.3$ (Petroleum Ether/ Ethyl Acetate: 2.5/7.5). ${ }^{1} \mathrm{H}$ NMR $\left(300 \mathrm{MHz}, \mathrm{CDCl}_{3}\right) \delta$ $8.33-8.23(\mathrm{~m}, 2 \mathrm{H}), 7.82-7.73(\mathrm{~m}, 2 \mathrm{H}), 7.48-7.40(\mathrm{~m}, 3 \mathrm{H}), 7.39-7.31$ $(\mathrm{m}, 2 \mathrm{H}), 4.34-4.17(\mathrm{~m}, 4 \mathrm{H}), 3.51-2.88(\mathrm{~m}, 3 \mathrm{H}), 2.44(\mathrm{~s}, 3 \mathrm{H}), 2.41-2.22$ $(\mathrm{m}, 2 \mathrm{H}) .{ }^{13} \mathrm{C} \mathrm{NMR}\left(75 \mathrm{MHz}, \mathrm{CDCl}_{3}\right) \delta 145.5,139.3,132.4,130.8,130.2$, 128.6, 128.0, 127.3, $122.4\left(\mathrm{t},{ }^{1} J_{C-F}=244.3 \mathrm{~Hz}\right), 63.9\left(\mathrm{dd},{ }^{3} J_{C-F}=5.7 \mathrm{~Hz}\right.$, $\left.{ }^{3} J_{C-F}=3.5 \mathrm{~Hz}\right), 63.4\left(\mathrm{t},{ }^{3} J_{C-F}=5.9 \mathrm{~Hz}\right), 36.3\left(\mathrm{t},{ }^{2} J_{C-F}=25.6 \mathrm{~Hz}\right), 34.8\left(\mathrm{t},{ }^{2} \mathrm{JC}_{\mathrm{C}}\right.$ $F=25.7 \mathrm{~Hz}), 31.3\left(\mathrm{t},{ }^{3} \mathrm{JC}_{-\mathrm{F}}=5.0 \mathrm{~Hz}\right), 21.8 .{ }^{19} \mathrm{~F}\{\mathrm{H}\} \mathrm{NMR}\left(471 \mathrm{MHz}, \mathrm{CDCl}_{3}\right)$ $\delta-104.66$ (AB system, ${ }^{2} J_{F-F}=250.8 \mathrm{~Hz}$ ), -106.45 (AB system, ${ }^{2} J_{F-F}=250.9$ $\mathrm{Hz})$. HRMS (ESI): calcd. for $[\mathrm{M}+\mathrm{Na}]^{+}\left(\mathrm{C}_{20} \mathrm{H}_{21} \mathrm{NO}_{4} \mathrm{~F}_{2} \mathrm{SNa}\right)=432.1052$; found: 432.1058 (2 ppm).

\section{3-(3-azido-1,1-difluoropropyl)-5-phenyl-3,4-dihydro-2H-pyrrole 1-} oxide 21a

To 3-(1,1-difluoro-3-(tosyloxy)propyl)-5-phenyl-3,4-dihydro-2H-pyrrole 1oxide $20 \mathrm{a}(60 \mathrm{mg}, 0.15 \mathrm{mmol})$, sodium azide $(15 \mathrm{mg}, 0.22 \mathrm{mmol}$ ) was added in $2 \mathrm{~mL}$ DMF. The reaction was monitored by TLC. At the end saturated $\mathrm{NH}_{4} \mathrm{Cl}(2 \mathrm{~mL})$ was added and the reaction mixture was extracted with DCM $(3 \times 5 \mathrm{~mL})$. The organic layers were separated, washed with water ( $1 \times 5 \mathrm{~mL}$ ), dried over $\mathrm{Na}_{2} \mathrm{SO}_{4}$ and concentrated under vacuum. After purification by chromatography on silica gel, 21a (35 mg, 85\%) was obtained as colorless oil $(75 \mathrm{mg}, 81 \%) . R_{f}=0.3$ (Petroleum Ether/ Ethyl Acetate: $2.5 / 7.5) . \mathrm{mp}=72{ }^{\circ} \mathrm{C} .{ }^{1} \mathrm{H} \mathrm{NMR}\left(300 \mathrm{MHz}, \mathrm{CDCl}_{3}\right) \delta 8.40-8.24(\mathrm{~m}$, $2 \mathrm{H}), 7.54-7.42(\mathrm{~m}, 3 \mathrm{H}), 4.39-4.32(\mathrm{~m}, 2 \mathrm{H}), 3.61\left(\mathrm{t},{ }^{3} \mathrm{JH}_{\mathrm{H}-\mathrm{H}}=6.8 \mathrm{~Hz}, 2 \mathrm{H}\right)$, $3.44-3.02(\mathrm{~m}, 3 \mathrm{H}), 2.30-2.11(\mathrm{~m}, 2 \mathrm{H}) .{ }^{13} \mathrm{C} \mathrm{NMR}\left(75 \mathrm{MHz}, \mathrm{CDCl}_{3}\right) \delta$ 139.5, 130.9, 128.7, 128.6, 127.4, $122.7\left(\mathrm{t},{ }^{1} J_{C-F}=244.2 \mathrm{~Hz}\right), 64.1\left(\mathrm{dd},{ }^{3} J_{C-}\right.$ $\left.F=5.5 \mathrm{~Hz},{ }^{3} J_{C-F}=3.9 \mathrm{~Hz}\right), 44.7\left(\mathrm{t},{ }^{3} J_{C-F}=5.3 \mathrm{~Hz}\right), 36.6\left(\mathrm{t},{ }^{2} J_{C-F}=25.9 \mathrm{~Hz}\right)$, 
$34.6\left(\mathrm{t},{ }^{2} J_{C-F}=25.2 \mathrm{~Hz}\right), 31.4\left(\mathrm{dd},{ }^{3} J_{C-F}=5.8 \mathrm{~Hz},{ }^{3} J_{C-F}=4.4 \mathrm{~Hz}\right) .{ }^{19} \mathrm{~F}\{\mathrm{H}\}$ $\operatorname{NMR}\left(471 \mathrm{MHz}, \mathrm{CDCl}_{3}\right) \delta-101.23\left(\mathrm{AB}\right.$ system, $\left.{ }^{2} J_{F-F}=253.9 \mathrm{~Hz}\right),-102.47$ (AB system, ${ }^{2} J_{F-F}=253.9 \mathrm{~Hz}$ ). HRMS (ESI): calcd. for $[\mathrm{M}+\mathrm{Na}]^{+}$ $\left(\mathrm{C}_{13} \mathrm{H}_{14} \mathrm{~N}_{4} \mathrm{OF}_{2} \mathrm{Na}\right)=303.1027$; found: 303.1027 (0 ppm).

\section{General procedure 4 for the synthesis of difluoropropargylic alcohols 23}

To a solution of $n$-butyllithium $2.5 \mathrm{M}(2.77 \mathrm{~mL}, 6.92 \mathrm{mmol})$ in anhydrous THF $\left(40 \mathrm{~mL}\right.$ ) cooled to $-90^{\circ} \mathrm{C}$, was added under nitrogen, a solution of 22 (500 mg, $2.10 \mathrm{mmol})$. The mixture was stirred for $30 \mathrm{~min}$ at $-90^{\circ} \mathrm{C}$. Then, aldehyde 2 (1.3 equiv) in anhydrous THF $(10 \mathrm{~mL})$ was added at $-90^{\circ} \mathrm{C}$, stirred for $45 \mathrm{~min}$, then the reaction mixture was allowed to warm to r.t. within $2 \mathrm{~h}$. The mixture was then treated with a saturated $\mathrm{NH}_{4} \mathrm{Cl}$ solution and extracted with ethyl acetate $(3 \times 40 \mathrm{~mL})$. The combined organic phases were washed with water, dried over $\mathrm{Na}_{2} \mathrm{SO}_{4}$ and concentrated under vacuum. After purification by chromatography on silica gel, the propargylic alcohols $\mathbf{2 3}$ were obtained.

\section{4,4-difluoro-1-(4-fluorophenyl)hept-2-yne-1,7-diol 23b}

Colorless oil, yield $380 \mathrm{mg}(70 \%) . R_{f}=0.2$ (Hexane / Ethyl Acetate: $7 / 3$ ). ${ }^{1} \mathrm{H}$ NMR $\left(300 \mathrm{MHz}, \mathrm{CDCl}_{3}\right) \delta 7.54-7.39(\mathrm{~m}, 2 \mathrm{H}), 7.12-7.01(\mathrm{~m}, 2 \mathrm{H})$, $5.48\left(\mathrm{t},{ }^{3} \mathrm{~J}_{\mathrm{H}-\mathrm{H}}=4.0 \mathrm{~Hz}, 1 \mathrm{H}\right), 3.68\left(\mathrm{t},{ }^{3} \mathrm{JH}-\mathrm{H}=6.3 \mathrm{~Hz}, 2 \mathrm{H}\right), 2.38-2.02(\mathrm{~m}, 3 \mathrm{H})$, $1.95-1.63(\mathrm{~m}, 2 \mathrm{H}) .{ }^{13} \mathrm{C} \mathrm{NMR}\left(75 \mathrm{MHz}, \mathrm{CDCl}_{3}\right) \delta 163.0\left(\mathrm{~d},{ }^{1} \mathrm{JC}_{\mathrm{C} F}=247.7\right.$ $\mathrm{Hz}), 135.0\left(\mathrm{dt},{ }^{4} J_{C-F}=3.3 \mathrm{~Hz},{ }^{5} J_{C-F}=1.6 \mathrm{~Hz}\right), 128.6\left(\mathrm{~d},{ }^{3} J_{C-F}=8.5 \mathrm{~Hz}\right)$, $115.9\left(\mathrm{~d},{ }^{2} J_{C-F}=21.8 \mathrm{~Hz}\right), 114.9\left(\mathrm{t},{ }^{1} J_{C-F}=233.0 \mathrm{~Hz}\right), 86.9\left(\mathrm{t},{ }^{3} J_{C-F}=6.8\right.$ $\mathrm{Hz}), 79.5\left(\mathrm{t},{ }^{2} J_{C-F}=41.2 \mathrm{~Hz}\right), 63.5\left(\mathrm{t},{ }^{4} J_{C-F}=2.0 \mathrm{~Hz}\right), 61.6,35.8\left(\mathrm{t},{ }^{2} J_{C-F}=\right.$ $26.4 \mathrm{~Hz}), 26.0\left(\mathrm{t},{ }^{3} J_{C-F}=3.4 \mathrm{~Hz}\right) \cdot{ }^{19} \mathrm{~F}\{\mathrm{H}\} \mathrm{NMR}\left(282 \mathrm{MHz}, \mathrm{CDCl}_{3}\right) \delta-83.16$ (s), -112.91 (s). HRMS (ESI): calcd. for $[\mathrm{M}+\mathrm{Na}]^{+}\left(\mathrm{C}_{13} \mathrm{H}_{13} \mathrm{O}_{2} \mathrm{~F}_{3} \mathrm{Na}\right)=$ 281.0760; found: 281.0762 (1 ppm)

\section{1-(4-chlorophenyl)-4,4-difluorohept-2-yne-1,7-diol 23c}

Yellow oil, yield $496 \mathrm{mg}(86 \%) . R_{f}=0.2$ (hexane / Ethyl Acetate: $7 / 3$ ). ${ }^{1} \mathrm{H}$ NMR $\left(300 \mathrm{MHz}, \mathrm{CDCl}_{3}\right) \delta 7.44-7.36(\mathrm{~m}, 2 \mathrm{H}), 7.36-7.30(\mathrm{~m}, 2 \mathrm{H}), 5.44$ (t, $\left.{ }^{3} \mathrm{H}-\mathrm{H}=4.0 \mathrm{~Hz}, 1 \mathrm{H}\right), 3.64\left(\mathrm{t},{ }^{3} \mathrm{JH}-\mathrm{H}=6.3 \mathrm{~Hz}, 2 \mathrm{H}\right), 3.38(\mathrm{bs}, 1 \mathrm{H}), 2.35-$ $2.05(\mathrm{~m}, 2 \mathrm{H}), 1.91-1.61(\mathrm{~m}, 2 \mathrm{H}) .{ }^{13} \mathrm{C}$ NMR $\left(75 \mathrm{MHz}, \mathrm{CDCl}_{3}\right) \delta 137.6$, 134.7, 129.0, 128.1, $114.8\left(\mathrm{t},{ }^{1} J_{C-F}=232.9 \mathrm{~Hz}\right), 86.8\left(\mathrm{t},{ }^{3} J_{C-F}=6.8 \mathrm{~Hz}\right), 79.4$ $\left(\mathrm{t},{ }^{2} J_{C-F}=41.1 \mathrm{~Hz}\right), 63.3\left(\mathrm{t},{ }^{4} J_{C-F}=1.9 \mathrm{~Hz}\right), 61.4,35.7\left(\mathrm{t},{ }^{2} J_{C-F}=26.4 \mathrm{~Hz}\right)$, $25.9\left(\mathrm{t},{ }^{3} \mathrm{~J}_{\mathrm{C}-\mathrm{F}}=3.5 \mathrm{~Hz}\right) .{ }^{19} \mathrm{~F}\{\mathrm{H}\} \mathrm{NMR}\left(282 \mathrm{MHz}, \mathrm{CDCl}_{3}\right) \delta-83.07$ (s). HRMS (ESI): calcd. for $[\mathrm{M}+\mathrm{Na}]^{+}\left(\mathrm{C}_{13} \mathrm{H}_{13} \mathrm{O}_{2} \mathrm{~F}_{2}{ }^{35} \mathrm{CINa}\right)=297.0464$; found: 297.0467 (1 ppm).

\section{4,4-difluoro-1-(furan-2-yl)hept-2-yne-1,7-diol 23d}

Black oil, yield $435 \mathrm{mg}(90 \%) . \mathrm{R}_{\mathrm{f}}=0.1$ (hexane / Ethyl Acetate: $\left.7 / 3\right) .{ }^{1} \mathrm{H}$ $\operatorname{NMR}\left(300 \mathrm{MHz}, \mathrm{CDCl}_{3}\right) \delta 7.43\left(\mathrm{dd},{ }^{3} \mathrm{JH}_{\mathrm{H}-\mathrm{H}}=1.8 \mathrm{~Hz},{ }^{4} \mathrm{~J}-\mathrm{H}=0.9 \mathrm{~Hz}, 1 \mathrm{H}\right), 6.46$ (dt, ${ }^{3} \mathrm{JH}_{\mathrm{H}-\mathrm{H}}=3.3 \mathrm{~Hz},{ }^{4} \mathrm{~J}_{\mathrm{H}-\mathrm{H}}=0.8 \mathrm{~Hz}, 1 \mathrm{H}$ ), 6.37 (dd, ${ }^{3} \mathrm{~J}_{\mathrm{H}-\mathrm{H}}=3.3 \mathrm{~Hz},{ }^{3} \mathrm{JH}_{\mathrm{H}-\mathrm{H}}=1.9$ $\mathrm{Hz}, 1 \mathrm{H}), 5.53\left(\mathrm{t},{ }^{3} J_{H-H}=3.9 \mathrm{~Hz}, 1 \mathrm{H}\right), 3.71\left(\mathrm{t},{ }^{3} \mathrm{JH}_{\mathrm{H}-\mathrm{H}}=6.3 \mathrm{~Hz}, 2 \mathrm{H}\right), 2.33-2.04$ (m, 3H), $1.94-1.75(\mathrm{~m}, 2 \mathrm{H}) .{ }^{13} \mathrm{C} \mathrm{NMR}\left(75 \mathrm{MHz}, \mathrm{CDCl}_{3}\right) \delta 151.5\left(\mathrm{t},{ }^{5} \mathrm{JC}-\mathrm{F}=\right.$ $1.9 \mathrm{~Hz}), 143.6,114.8\left(\mathrm{t},{ }^{1} J_{C-F}=233.1 \mathrm{~Hz}\right), 110.7,108.5,84.7\left(\mathrm{t},{ }^{3} J_{C-F}=6.8\right.$ $\mathrm{Hz}), 78.5\left(\mathrm{t},{ }^{2} J_{C-F}=41.3 \mathrm{~Hz}\right), 61.6,57.8\left(\mathrm{t},{ }^{4} J_{C-F}=1.8 \mathrm{~Hz}\right), 35.8\left(\mathrm{t},{ }^{2} J_{C-F}=\right.$ $26.3 \mathrm{~Hz}), 26.0\left(\mathrm{t},{ }^{3} J_{C-F}=3.5 \mathrm{~Hz}\right) .{ }^{19} \mathrm{~F}\{\mathrm{H}\} \mathrm{NMR}\left(282 \mathrm{MHz}, \mathrm{CDCl}_{3}\right) \delta-83.55$. HRMS (ESI): calcd. for $[\mathrm{M}+\mathrm{Na}]^{+}\left(\mathrm{C}_{11} \mathrm{H}_{12} \mathrm{O}_{3} \mathrm{~F}_{2} \mathrm{Na}\right)=253.0647$; found: 253.0647 (0 ppm).

\section{4,4-difluoro-1-(4-methoxyphenyl)hept-2-yne-1,7-diol 23e}

Colorless oil, yield $540 \mathrm{mg}(95 \%) . \mathrm{R}_{\mathrm{f}}=0.3$ (Petroleum Ether / Ethyl Acetate: 6/4). ${ }^{1} \mathrm{H}$ NMR $\left(300 \mathrm{MHz}, \mathrm{CDCl}_{3}\right) \delta 7.50-7.35(\mathrm{~m}, 2 \mathrm{H}), 6.97-$ $6.87(\mathrm{~m}, 2 \mathrm{H}), 5.48\left(\mathrm{t},{ }^{3} \mathrm{JH}_{\mathrm{H} H}=4.0 \mathrm{~Hz}, 1 \mathrm{H}\right), 3.82(\mathrm{~s}, 3 \mathrm{H}), 3.71\left(\mathrm{t},{ }^{3} \mathrm{~J}_{\mathrm{H}-\mathrm{H}}=6.3\right.$ $\mathrm{Hz}, 2 \mathrm{H}), 2.32-2.06$ (m, 2H), 1.90 (bs, 2H, OH), $1.89-1.76(\mathrm{~m}, 2 \mathrm{H}) .{ }^{13} \mathrm{C}$ $\mathrm{NMR}\left(75 \mathrm{MHz}, \mathrm{CDCl}_{3}\right) \delta 160.2,131.4,128.3,114.9\left(\mathrm{t},{ }^{1} \mathrm{JC}_{\mathrm{F}-\mathrm{F}}=232.8 \mathrm{~Hz}\right)$, $114.3,87.1\left(\mathrm{t},{ }^{3} J_{C-F}=6.8 \mathrm{~Hz}\right), 79.3\left(\mathrm{t},{ }^{2} J_{C-F}=41.0 \mathrm{~Hz}\right), 64.0\left(\mathrm{t},{ }^{4} J_{C-F}=2.0\right.$ $\mathrm{Hz}), 61.7,55.5,35.9\left(\mathrm{t},{ }^{2} J_{C-F}=26.4 \mathrm{~Hz}\right), 26.1$ (t, $\left.{ }^{3} J_{C-F}=3.5 \mathrm{~Hz}\right) .{ }^{19} \mathrm{~F}\{\mathrm{H}\}$ $\operatorname{NMR}\left(471 \mathrm{MHz}, \mathrm{CDCl}_{3}\right) \delta-83.13$ (s). HRMS (ESI): calcd. for [M+Na] ${ }^{+}$ $\left(\mathrm{C}_{14} \mathrm{H}_{16} \mathrm{O}_{3} \mathrm{~F}_{2} \mathrm{Na}\right)=293.0960$; found: 293.0963 (1 ppm).

\section{General procedure 5 for the synthesis of bromo- compounds} 24

Representative procedure: 7-bromo-4,4-difluoro-1-(4fluorophenyl)hept-2-yn-1-ol 24b

Triphenylphosphine $(460 \mathrm{mg}, 1.76 \mathrm{mmol})$ in dichloromethane $(5 \mathrm{~mL})$ was added dropwise to a vigorously stirred mixture of alcohol 23b (350 mg, $1.36 \mathrm{mmol}$ ) with carbon tetrabromide $(585 \mathrm{mg}, 1.76 \mathrm{mmol})$ in dichloromethane $(10 \mathrm{~mL})$ at $0{ }^{\circ} \mathrm{C}$ under nitrogen. Petroleum ether was added, and the precipitate was then filtered off. Brominated compound $\mathbf{2 4 b}$ was separated by column chromatography to obtain a yellow oil (383 mg, $88 \%) . R_{f}=0.6$ (Hexane / Ethyl Acetate: $\left.8 / 2\right) .{ }^{1} \mathrm{H} \mathrm{NMR}\left(300 \mathrm{MHz}, \mathrm{CDCl}_{3}\right) \delta$ $7.55-7.41(\mathrm{~m}, 2 \mathrm{H}), 7.19-6.99(\mathrm{~m}, 2 \mathrm{H}), 5.52\left(\mathrm{t},{ }^{3} \mathrm{JH}-\mathrm{H}=3.9 \mathrm{~Hz}, 1 \mathrm{H}\right), 3.45$ (t, $\left.{ }^{3} \mathrm{H}-\mathrm{H}=6.3 \mathrm{~Hz}, 2 \mathrm{H}\right), 2.69$ (bs, $\left.1 \mathrm{H}\right), 2.50-1.92(\mathrm{~m}, 4 \mathrm{H}) .{ }^{13} \mathrm{C}$ NMR $(75$ $\left.\mathrm{MHz}, \mathrm{CDCl}_{3}\right) \delta 163.1\left(\mathrm{~d},{ }^{1} J_{C-F}=248.0 \mathrm{~Hz}\right), 134.8\left(\mathrm{dt},{ }^{4} J_{C-F}=3.3 \mathrm{~Hz},{ }^{5} \mathrm{~J}_{\mathrm{C}-\mathrm{F}}\right.$ $=1.6 \mathrm{~Hz}), 128.6\left(\mathrm{~d},{ }^{3} J_{C-F}=8.4 \mathrm{~Hz}\right), 115.9\left(\mathrm{~d},{ }^{2}{ }^{J} \mathrm{C}-\mathrm{F}=21.7 \mathrm{~Hz}\right), 114.2(\mathrm{t}$, $\left.{ }^{1} J_{C-F}=233.6 \mathrm{~Hz}\right), 86.9\left(\mathrm{t},{ }^{3} J_{C-F}=6.7 \mathrm{~Hz}\right), 79.3\left(\mathrm{t},{ }^{2} J_{C-F}=41.0 \mathrm{~Hz}\right), 63.6(\mathrm{t}$, $\left.{ }^{4} J_{C-F}=1.9 \mathrm{~Hz}\right), 37.9\left(\mathrm{t},{ }^{2} J_{C-F}=26.6 \mathrm{~Hz}\right), 32.2,26.2\left(\mathrm{t},{ }^{3} J_{C-F}=3.7 \mathrm{~Hz}\right) .{ }^{19} \mathrm{~F}$ $\{\mathrm{H}\} \mathrm{NMR}\left(282 \mathrm{MHz}, \mathrm{CDCl}_{3}\right) \delta-83.23$ (s), -112.63(s). HRMS (ESI): calcd. for $[\mathrm{M}+\mathrm{Na}]^{+}\left(\mathrm{C}_{13} \mathrm{H}_{12} \mathrm{OF}_{3}{ }^{79} \mathrm{BrNa}\right)=342.9915$; found: 342.9915 (0 ppm).

\section{7-bromo-1-(4-chlorophenyl)-4,4-difluorohept-2-yn-1-ol 24c}

Yellow oil, yield $262 \mathrm{mg}(61 \%)$ from $350 \mathrm{mg}(1.27 \mathrm{mmol})$ of $23 \mathrm{c}$. $R_{\mathrm{f}}=0.6$ (Hexane / Ethyl Acetate: 8/2). ${ }^{1} \mathrm{H}$ NMR $\left(300 \mathrm{MHz}, \mathrm{CDCl}_{3}\right) \delta 7.50-7.41(\mathrm{~m}$, 2H), $7.41-7.27(\mathrm{~m}, 2 \mathrm{H}), 5.52\left(\mathrm{t},{ }^{3} \mathrm{~J} H-H=3.2 \mathrm{~Hz}, 1 \mathrm{H}\right), 3.45\left(\mathrm{t},{ }^{3} \mathrm{JH}-\mathrm{H}=6.3\right.$ $\mathrm{Hz}, 2 \mathrm{H}), 2.70$ (bs, $1 \mathrm{H}), 2.38-2.20(\mathrm{~m}, 2 \mathrm{H}), 2.16-1.99(\mathrm{~m}, 2 \mathrm{H}) .{ }^{13} \mathrm{C} N M R$ $\left(75 \mathrm{MHz}, \mathrm{CDCl}_{3}\right) \delta 137.5\left(\mathrm{t},{ }^{5} \mathrm{~J}_{\mathrm{C}-\mathrm{F}}=1.5 \mathrm{~Hz}\right), 134.9,129.2,128.1,114.2(\mathrm{t}$, $\left.{ }^{1} J_{C-F}=233.6 \mathrm{~Hz}\right), 86.7\left(\mathrm{t},{ }^{3} J_{C-F}=6.7 \mathrm{~Hz}\right), 79.4\left(\mathrm{t},{ }^{2} J_{C-F}=41.0 \mathrm{~Hz}\right), 63.6(\mathrm{t}$, $\left.{ }^{4} J_{C-F}=1.9 \mathrm{~Hz}\right), 37.9\left(\mathrm{t},{ }^{2} J_{C-F}=26.6 \mathrm{~Hz}\right), 32.2,26.1\left(\mathrm{t},{ }^{3 \mathrm{j}} J_{C-F}=3.7 \mathrm{~Hz}\right) .{ }^{19} \mathrm{~F}$ $\{\mathrm{H}\} \mathrm{NMR}\left(282 \mathrm{MHz}, \mathrm{CDCl}_{3}\right) \delta-83.28$ (s). HRMS (ESI): calcd. for [M+Na] ${ }^{+}$ $\left(\mathrm{C}_{13} \mathrm{H}_{12} \mathrm{OF}_{2}{ }^{35} \mathrm{Cl}^{79} \mathrm{BrNa}\right)=358.9620$; found: 358.9621 (0 ppm).

\section{7-bromo-4,4-difluoro-1-(furan-2-yl)hept-2-yn-1-ol 24d}

Black oil, yield $227 \mathrm{mg}(51 \%)$ from $350 \mathrm{mg}(1.52 \mathrm{mmol})$ of $23 \mathrm{~d}$. $\mathrm{R}_{\mathrm{f}}=0.5$ (Hexane / Ethyl Acetate: 8/2). ${ }^{1} \mathrm{H}$ NMR $\left(300 \mathrm{MHz}, \mathrm{CDCl}_{3}\right) \delta 7.44$ (dd, ${ }^{3} \mathrm{~J}_{\mathrm{H}-\mathrm{H}}$ $\left.=1.9 \mathrm{~Hz},{ }^{4} \mathrm{~J}_{\mathrm{H}-\mathrm{H}}=0.9 \mathrm{~Hz}, 1 \mathrm{H}\right), 6.47\left(\mathrm{dt},{ }^{3} \mathrm{~J}_{\mathrm{H}-\mathrm{H}}=3.3 \mathrm{~Hz},{ }^{4} \mathrm{~J}_{\mathrm{H}-\mathrm{H}}=0.8 \mathrm{~Hz}, 1 \mathrm{H}\right)$, $6.38\left(\mathrm{dd},{ }^{3} \mathrm{~J}_{\mathrm{H}-\mathrm{H}}=3.3 \mathrm{~Hz},{ }^{3} \mathrm{~J}_{\mathrm{H}-\mathrm{H}}=1.8 \mathrm{~Hz}, 1 \mathrm{H}\right), 5.54\left(\mathrm{t},{ }^{3} \mathrm{~J}_{\mathrm{H}-\mathrm{H}}=3.9 \mathrm{~Hz}, 1 \mathrm{H}\right)$, $3.46\left(\mathrm{t},{ }^{3} \mathrm{JH}_{\mathrm{H}-\mathrm{H}}=6.2 \mathrm{~Hz}, 2 \mathrm{H}\right), 2.81$ (bs, $\left.1 \mathrm{H}\right), 2.42-2.02(\mathrm{~m}, 4 \mathrm{H}) .{ }^{13} \mathrm{C} \mathrm{NMR}$ $\left(75 \mathrm{MHz}, \mathrm{CDCl}_{3}\right) \delta 151.2\left(\mathrm{t},{ }^{5} \mathrm{~J}_{\mathrm{C}-\mathrm{F}}=2.0 \mathrm{~Hz}\right), 143.6,114.1\left(\mathrm{t},{ }^{1} \mathrm{~J}_{\mathrm{C}-F}=233.6\right.$ $\mathrm{Hz}), 110.7,108.6,84.8\left(\mathrm{t},{ }^{3} J_{C-F}=6.9 \mathrm{~Hz}\right), 78.3\left(\mathrm{t},{ }^{2} J_{C-F}=41.1 \mathrm{~Hz}\right), 57.8(\mathrm{t}$, $\left.{ }^{4} J_{C-F}=2.0 \mathrm{~Hz}\right), 37.9\left(\mathrm{t},{ }^{2} J_{C-F}=26.5 \mathrm{~Hz}\right), 32.2,26.1\left(\mathrm{t},{ }^{3} J_{C-F}=3.8 \mathrm{~Hz}\right) .{ }^{19} \mathrm{~F}$ $\{\mathrm{H}\} \mathrm{NMR}\left(282 \mathrm{MHz}, \mathrm{CDCl}_{3}\right) \delta-83.5$ (s). HRMS (ESI): calcd. for $[\mathrm{M}+\mathrm{H}]^{+}$ $\left(\mathrm{C}_{11} \mathrm{H}_{12} \mathrm{O}_{2} \mathrm{~F}_{2}{ }^{81} \mathrm{Br}\right)=294.9962$; found: 294.9962 (0 ppm).

\section{7-bromo-4,4-difluoro-1-(4-methoxyphenyl)hept-2-yn-1-ol 24e}

Pale yellow oil, yield $332 \mathrm{mg}(77 \%)$ from $350 \mathrm{mg}(1.29 \mathrm{mmol})$ of $23 \mathrm{e} . \mathrm{R}_{\mathrm{f}}=$ 0.4 (Petroleum Ether / Ethyl Acetate: 8/2). ${ }^{1} \mathrm{H}$ NMR $(300 \mathrm{MHz}$, Chloroformd) $\delta 7.46-7.39(\mathrm{~m}, 2 \mathrm{H}), 6.96-6.89(\mathrm{~m}, 2 \mathrm{H}), 5.49\left(\mathrm{t},{ }^{3} \mathrm{~J}_{\mathrm{H}-\mathrm{H}}=4.0 \mathrm{~Hz}, 1 \mathrm{H}\right)$, $3.82(\mathrm{~s}, 3 \mathrm{H}), 3.46\left(\mathrm{t},{ }^{3} \mathrm{JH}_{\mathrm{H}-\mathrm{H}}=6.3 \mathrm{~Hz}, 2 \mathrm{H}\right), 2.36-2.07(\mathrm{~m}, 5 \mathrm{H}) .{ }^{13} \mathrm{C}$ NMR $(75$ $\mathrm{MHz}$, Chloroform- $d$ ) $\delta 160.2,131.3\left(\mathrm{t},{ }^{5} \mathrm{~J}_{\mathrm{C}-F}=1.6 \mathrm{~Hz}\right), 128.3,114.4,114.3$ $\left(\mathrm{t},{ }^{1} J_{C-F}=233.3 \mathrm{~Hz}\right), 87.4\left(\mathrm{t},{ }^{3} J_{C-F}=6.7 \mathrm{~Hz}\right), 79.0\left(\mathrm{t},{ }^{2} J_{C-F}=40.8 \mathrm{~Hz}\right), 64.0$ $\left(\mathrm{t},{ }^{4} J_{C-F}=2.0 \mathrm{~Hz}\right), 55.5,38.0\left(\mathrm{t},{ }^{2} J_{C-F}=26.7 \mathrm{~Hz}\right), 32.3,26.2\left(\mathrm{t},{ }^{3} J_{C-F}=3.7\right.$ $\mathrm{Hz}) .{ }^{19} \mathrm{~F}\{\mathrm{H}\} \mathrm{NMR}\left(471 \mathrm{MHz}, \mathrm{CDCl}_{3}\right) \delta-83.08$ (s). HRMS (ESI): calcd. for $[\mathrm{M}+\mathrm{Na}]^{+}\left(\mathrm{C}_{14} \mathrm{H}_{15} \mathrm{O}_{2} \mathrm{~F}_{2}{ }^{81} \mathrm{BrNa}\right)=357.0095$; found: 357.0095 (0 ppm).

\section{General procedure 6 for the synthesis of cyclic nitro compounds 25}

Representative procedure: 2-(2,2-difluoro-6-nitrocyclohexyl)-1phenylethan-1-one 25a

To the brominated compound 24a (200 $\mathrm{mg}, 0.66 \mathrm{mmol}$ ) was added nitromethane $(71 \mu \mathrm{l}, 1.31 \mathrm{mmol})$ and DBU (395 $\mu \mathrm{l}, 2.63 \mathrm{mmol})$ in THF (3 $\mathrm{ml})$. The reaction mixture was stirred at room temperature overnight. At the end saturated $\mathrm{NH}_{4} \mathrm{Cl}(10 \mathrm{~mL})$ was added and the reaction mixture was extracted with ethyl acetate $(3 \times 20 \mathrm{~mL})$. The organic layers were separated, 
washed with water $(1 \times 10 \mathrm{~mL})$, dried over $\mathrm{Na}_{2} \mathrm{SO}_{4}$ and concentrated under vacuum. After purification by chromatography on silica gel, 25a (142 mg, $76 \%$ ) was obtained as white solid. $R_{f}=0.5$ (Hexane / Ethyl Acetate: 9/1). $\mathrm{mp}=93^{\circ} \mathrm{C} .{ }^{1} \mathrm{H} \mathrm{NMR}\left(300 \mathrm{MHz}, \mathrm{CDCl}_{3}\right) \delta 8.02-7.86(\mathrm{~m}, 2 \mathrm{H}), 7.64-7.52$ $(\mathrm{m}, 1 \mathrm{H}), 7.52-7.39(\mathrm{~m}, 2 \mathrm{H}), 4.55\left(\mathrm{td},{ }^{3} \mathrm{~J}_{\mathrm{H}-\mathrm{H}}=11.6 \mathrm{~Hz},{ }^{3} \mathrm{~J}_{\mathrm{H}-\mathrm{H}}=4.0 \mathrm{~Hz}, 1 \mathrm{H}\right)$, $3.56\left(\mathrm{dd},{ }^{2} \mathrm{JH}_{\mathrm{H}-\mathrm{H}}=17.8 \mathrm{~Hz},{ }^{3} \mathrm{JH}-\mathrm{H}=5.6 \mathrm{~Hz}, 1 \mathrm{H}\right), 3.51-3.36(\mathrm{~m}, 1 \mathrm{H}), 2.97$ $\left(\mathrm{dd},{ }^{2} \mathrm{~J}_{\mathrm{H}-\mathrm{H}}=17.8 \mathrm{~Hz},{ }^{3} \mathrm{JH}_{\mathrm{H} H}=4.3 \mathrm{~Hz}, 1 \mathrm{H}\right), 2.43-1.61(\mathrm{~m}, 6 \mathrm{H}) .{ }^{13} \mathrm{C}$ NMR $(75$ $\left.\mathrm{MHz}, \mathrm{CDCl}_{3}\right) \delta 195.8,136.4,133.5,128.8,128.3,122.9\left(\mathrm{dd},{ }^{1} \mathrm{~J}-\mathrm{F}=249.5\right.$ $\left.\mathrm{Hz},{ }^{1} J_{C-F}=242.4 \mathrm{~Hz}\right), 87.0\left(\mathrm{~d},{ }^{3} J_{C-F}=8.5 \mathrm{~Hz}\right), 42.6\left(\mathrm{dd},{ }^{2} J_{C-F}=24.4,{ }^{2} J_{C-}\right.$ $F=22.2 \mathrm{~Hz}), 34.0\left(\mathrm{t},{ }^{3} J_{C-F}=2.4 \mathrm{~Hz}\right), 33.2\left(\mathrm{dd},{ }^{2} J_{C-F}=25.0 \mathrm{~Hz},{ }^{2} J_{C-F}=22.3\right.$ $\mathrm{Hz}), 30.2\left(\mathrm{~d},{ }^{4} J_{C-F}=1.6 \mathrm{~Hz}\right), 19.3\left(\mathrm{~d},{ }^{3} J_{C-F}=10.2 \mathrm{~Hz}\right) .{ }^{19} \mathrm{~F}\{\mathrm{H}\}$ NMR $(282$ $\mathrm{MHz}, \mathrm{CDCl}_{3}$ ) $\delta-95.5\left(\mathrm{AB}\right.$ system, ${ }^{2} \mathrm{JF}_{-F}=246.6 \mathrm{~Hz}$ ), -108.2 (AB system, $\left.{ }^{2} J_{F-F}=246.6 \mathrm{~Hz}\right)$. HRMS (ESI): calcd. for $[\mathrm{M}+\mathrm{Na}]^{+}\left(\mathrm{C}_{14} \mathrm{H}_{15} \mathrm{NO}_{3} \mathrm{~F}_{2} \mathrm{Na}\right)=$ 306.0912; found: 306.0914 (1 ppm).

2-(2,2-difluoro-6-nitrocyclohexyl)-1-(4-fluorophenyl)ethan-1-one 25b White solid, yield $124 \mathrm{mg}(66 \%)$ from $200 \mathrm{mg}(0.62 \mathrm{mmol})$ of $24 \mathrm{~b}$. $\mathrm{R}_{\mathrm{f}}=0.5$ (Hexane / Ethyl Acetate: 9/1). $\mathrm{mp}=89^{\circ} \mathrm{C} .{ }^{1} \mathrm{H}$ NMR $\left(300 \mathrm{MHz}, \mathrm{CDCl}_{3}\right) \delta$ $8.04-7.90(\mathrm{~m}, 2 \mathrm{H}), 7.19-7.00(\mathrm{~m}, 2 \mathrm{H}), 4.53\left(\mathrm{td},{ }^{3} \mathrm{JH}_{\mathrm{H} H}=11.6 \mathrm{~Hz},{ }^{3} \mathrm{JH}_{\mathrm{H}-\mathrm{H}}=\right.$ $4.0 \mathrm{~Hz}, 1 \mathrm{H}), 3.51\left(\mathrm{dd},{ }^{2} \mathrm{~J}_{\mathrm{H}-\mathrm{H}}=17.6 \mathrm{~Hz},{ }^{3} \mathrm{JH}-\mathrm{H}=5.5 \mathrm{~Hz}, 1 \mathrm{H}\right), 3.47-3.32(\mathrm{~m}$, $1 \mathrm{H}), 2.94\left(\mathrm{dd},{ }^{2} \mathrm{~J}_{\mathrm{H}-\mathrm{H}}=17.6 \mathrm{~Hz},{ }^{3} \mathrm{~J}_{\mathrm{H}-\mathrm{H}}=4.4 \mathrm{~Hz}, 1 \mathrm{H}\right), 2.42-1.59(\mathrm{~m}, 6 \mathrm{H}) .{ }^{13} \mathrm{C}$ $\operatorname{NMR}\left(75 \mathrm{MHz}, \mathrm{CDCl}_{3}\right) \delta 194.2,166.0\left(\mathrm{~d},{ }^{1} J_{C-F}=255.3 \mathrm{~Hz}\right), 132.8\left(\mathrm{~d},{ }^{4} J_{C-F}\right.$ $=3.1 \mathrm{~Hz}), 131.0\left(\mathrm{~d},{ }^{3} J_{C-F}=9.5 \mathrm{~Hz}\right), 122.9\left(\mathrm{dd},{ }^{1} J_{C-F}=249.4 \mathrm{~Hz},{ }^{1} J_{C-F}=\right.$ $242.5 \mathrm{~Hz}), 115.9\left(\mathrm{~d},{ }^{2} J_{C-F}=22.0 \mathrm{~Hz}\right), 86.9\left(\mathrm{~d},{ }^{3} J_{C-F}=8.4 \mathrm{~Hz}\right), 42.7\left(\mathrm{dd},{ }^{2} J_{C}-\right.$ $\left.F=24.4 \mathrm{~Hz},{ }^{2} J_{C-F}=22.2 \mathrm{~Hz}\right), 33.9\left(\mathrm{t},{ }^{3} J_{C-F}=2.4 \mathrm{~Hz}\right), 33.2\left(\mathrm{dd},{ }^{2} J_{C-F}=24.9\right.$ $\left.\mathrm{Hz},{ }^{2} J_{C-F}=22.2 \mathrm{~Hz}\right), 30.1\left(\mathrm{~d},{ }^{4} J_{C-F}=1.7 \mathrm{~Hz}\right), 19.2\left(\mathrm{~d},{ }^{3} J_{C-F}=10.2 \mathrm{~Hz}\right) .{ }^{19} \mathrm{~F}$ $\{\mathrm{H}\} \operatorname{NMR}\left(282 \mathrm{MHz}, \mathrm{CDCl}_{3}\right) \delta-95.51\left(\mathrm{~d},{ }^{2} J_{F-F}=246.3 \mathrm{~Hz}\right),-104.61(\mathrm{~s})$, $108.22\left(\mathrm{~d},{ }^{2} J_{F-F}=246.4 \mathrm{~Hz}\right)$. HRMS (ESI): calcd. for $[\mathrm{M}+\mathrm{Na}]^{+}$ $\left(\mathrm{C}_{14} \mathrm{H}_{14} \mathrm{NO}_{3} \mathrm{~F}_{3} \mathrm{Na}\right)=324.0818$; found: 324.0822 (1 ppm).

1-(4-chlorophenyl)-2-(2,2-difluoro-6-nitrocyclohexyl)ethan-1-one 25c White solid, yield $132 \mathrm{mg}(70 \%)$ from $200 \mathrm{mg}(0.59 \mathrm{mmol})$ of $24 \mathrm{c}$. $R_{\mathrm{f}}=0.5$ (Hexane / Ethyl Acetate: 9/1). $\mathrm{mp}=98{ }^{\circ} \mathrm{C} .{ }^{1} \mathrm{H}$ NMR $\left(300 \mathrm{MHz}, \mathrm{CDCl}_{3}\right) \delta$ $7.91-7.83(\mathrm{~m}, 2 \mathrm{H}), 7.47-7.39(\mathrm{~m}, 2 \mathrm{H}), 4.52\left(\mathrm{td},{ }^{3} \mathrm{~J}_{\mathrm{H}-\mathrm{H}}=11.7 \mathrm{~Hz},{ }^{3} \mathrm{JH}_{\mathrm{H} H}=\right.$ $4.1 \mathrm{~Hz}, 1 \mathrm{H}), 3.50\left(\mathrm{dd},{ }^{2} \mathrm{JH}_{\mathrm{H} H}=17.6 \mathrm{~Hz},{ }^{3} \mathrm{JH}_{\mathrm{H}-\mathrm{H}}=5.5 \mathrm{~Hz}, 1 \mathrm{H}\right), 3.46-3.32(\mathrm{~m}$, $1 \mathrm{H}), 2.94\left(\mathrm{dd},{ }^{2} \mathrm{JH}_{\mathrm{H}-\mathrm{H}}=17.6 \mathrm{~Hz},{ }^{3} \mathrm{~J}_{\mathrm{H}-\mathrm{H}}=4.3 \mathrm{~Hz}, 1 \mathrm{H}\right), 2.40-1.62(\mathrm{~m}, 6 \mathrm{H}) .{ }^{13} \mathrm{C}$ $\operatorname{NMR}\left(75 \mathrm{MHz}, \mathrm{CDCl}_{3}\right) \delta 194.7,140.0,134.7,129.7,129.1,122.9$ (dd, ${ }^{1} J_{C}$ $\left.F=249.4 \mathrm{~Hz},{ }^{1} J_{C-F}=242.4 \mathrm{~Hz}\right), 86.9\left(\mathrm{~d},{ }^{3} J_{C-F}=8.4 \mathrm{~Hz}\right), 42.7\left(\mathrm{dd},{ }^{2} J_{C-F}=\right.$ $\left.24.4 \mathrm{~Hz},{ }^{2} J_{C-F}=22.2 \mathrm{~Hz}\right), 33.9\left(\mathrm{t},{ }^{3} J_{C-F}=2.4 \mathrm{~Hz}\right), 33.2\left(\mathrm{dd},{ }^{2} J_{C-F}=25.0 \mathrm{~Hz}\right.$, $\left.{ }^{2} J_{C-F}=22.2 \mathrm{~Hz}\right), 30.2\left(\mathrm{~d},{ }^{4} J_{C-F}=1.6 \mathrm{~Hz}\right), 19.2\left(\mathrm{~d},{ }^{3} J_{C-F}=10.3 \mathrm{~Hz}\right) .{ }^{19} \mathrm{~F}\{\mathrm{H}\}$ NMR $\left(282 \mathrm{MHz}, \mathrm{CDCl}_{3}\right) \delta-95.43\left(\mathrm{AB}\right.$ system, $\left.{ }^{2} \mathrm{~J}_{F-F}=246.4 \mathrm{~Hz}\right),-108.22$ (AB system, ${ }^{2} J_{F-F}=246.4 \mathrm{~Hz}$ ). HRMS (ESI): calcd. for $[\mathrm{M}+\mathrm{Na}]^{+}$ $\left(\mathrm{C}_{14} \mathrm{H}_{14} \mathrm{NO}_{3} \mathrm{~F}_{2}{ }^{35} \mathrm{CINa}\right)=340.0522$; found: 340.0520 (1 ppm).

\section{2-(2,2-difluoro-6-nitrocyclohexyl)-1-(furan-2-yl)ethan-1-one 25d} White solid, yield $97 \mathrm{mg}(52 \%)$ from $200 \mathrm{mg}(0.68 \mathrm{mmol})$ of $24 \mathrm{~d}$. $\mathrm{R}_{\mathrm{f}}=0.3$ (Hexane / Ethyl Acetate: 9/1). mp $=98{ }^{\circ} \mathrm{C} .{ }^{1} \mathrm{H}$ NMR $\left(300 \mathrm{MHz}, \mathrm{CDCl}_{3}\right) \delta$ $7.58\left(\mathrm{dd},{ }^{3} \mathrm{~J}_{\mathrm{H}-\mathrm{H}}=1.7 \mathrm{~Hz},{ }^{4} \mathrm{~J}_{\mathrm{H}-\mathrm{H}}=0.8 \mathrm{~Hz}, 1 \mathrm{H}\right), 7.22\left(\mathrm{dd},{ }^{3} \mathrm{~J}_{\mathrm{H}-\mathrm{H}}=3.6 \mathrm{~Hz},{ }^{4} \mathrm{~J}_{\mathrm{H}-\mathrm{H}}\right.$

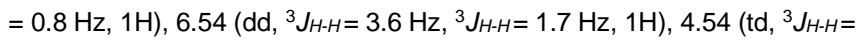
$\left.11.5 \mathrm{~Hz},{ }^{3} \mathrm{H}-\mathrm{H}=4.0 \mathrm{~Hz}, 1 \mathrm{H}\right), 3.49-3.19(\mathrm{~m}, 2 \mathrm{H}), 2.89-2.76(\mathrm{~m}, 1 \mathrm{H}), 2.40$ $-1.54(\mathrm{~m}, 6 \mathrm{H}) .{ }^{13} \mathrm{C}$ NMR $\left(75 \mathrm{MHz}, \mathrm{CDCl}_{3}\right) \delta 184.8,152.1,146.7,122.8$ $\left(\mathrm{dd},{ }^{1} J_{C-F}=249.4 \mathrm{~Hz},{ }^{1} J_{C-F}=242.5 \mathrm{~Hz}\right), 117.6,112.6,86.9\left(\mathrm{~d},{ }^{3} J_{C-F}=8.4\right.$ $\mathrm{Hz}), 42.2\left(\mathrm{dd},{ }^{2} J_{C-F}=24.5 \mathrm{~Hz},{ }^{2} J_{C-F}=22.3 \mathrm{~Hz}\right), 33.6\left(\mathrm{t},{ }^{3} J_{C-F}=2.5 \mathrm{~Hz}\right), 33.2$ $\left(\mathrm{dd},{ }^{2} J_{C-F}=25.0 \mathrm{~Hz},{ }^{2} J_{C-F}=22.3 \mathrm{~Hz}\right), 30.3\left(\mathrm{~d},{ }^{4} J_{C-F}=1.5 \mathrm{~Hz}\right), 19.3\left(\mathrm{~d},{ }^{3} J_{C-F}\right.$ $=10.2 \mathrm{~Hz}) \cdot{ }^{19} \mathrm{~F}\{\mathrm{H}\} \mathrm{NMR}\left(282 \mathrm{MHz}, \mathrm{CDCl}_{3}\right) \delta-95.46\left(\mathrm{AB}\right.$ system, ${ }^{2} \mathrm{~J}_{F-F}=$ $246.8 \mathrm{~Hz}),-108.40\left(\mathrm{~d},{ }^{2} J_{F-F}=246.8 \mathrm{~Hz}\right)$. HRMS $(\mathrm{ESI})$ : calcd. for $[\mathrm{M}+\mathrm{Na}]^{+}$ $\left(\mathrm{C}_{12} \mathrm{H}_{13} \mathrm{NO}_{4} \mathrm{~F}_{2} \mathrm{Na}\right)=296.0705$; found: 296.0708 (1 ppm).

\section{2-(2,2-difluoro-6-nitrocyclohexyl)-1-(4-methoxyphenyl)ethan-1-one} $25 \mathrm{e}$

White solid, yield $92 \mathrm{mg}(49 \%)$ from $200 \mathrm{mg}(0.60 \mathrm{mmol})$ of $24 \mathrm{e} . \mathrm{R}_{\mathrm{f}}=0.4$ (Petroleum Ether / Ethyl Acetate: $8 / 2) . \mathrm{mp}=125^{\circ} \mathrm{C} .{ }^{1} \mathrm{H}$ NMR $(300 \mathrm{MHz}$, $\left.\mathrm{CDCl}_{3}\right) \delta=7.97-7.89(\mathrm{~m}, 2 \mathrm{H}), 6.99-6.86(\mathrm{~m}, 2 \mathrm{H}), 4.61-4.45(\mathrm{~m}, 1 \mathrm{H})$, $3.86(\mathrm{~s}, 3 \mathrm{H}), 3.58-3.29(\mathrm{~m}, 2 \mathrm{H}), 2.42-1.52(\mathrm{~m}, 6 \mathrm{H})$. NMR $(75 \mathrm{MHz}$,
$\left.\mathrm{CDCl}_{3}\right) \delta 194.2,163.8,130.6,129.4,122.9\left(\mathrm{dd},{ }^{1} J_{C-F}=249.7 \mathrm{~Hz},{ }^{1} J_{C-F}=\right.$ $249.5 \mathrm{~Hz}), 113.9,87.0\left(\mathrm{~d},{ }^{3} J_{C-F}=8.4 \mathrm{~Hz}\right), 55.6,42.6\left(\mathrm{dd},{ }^{2} J_{C-F}=24.4 \mathrm{~Hz}\right.$, $\left.{ }^{2} J_{C-F}=22.2 \mathrm{~Hz}\right), 33.5\left(\mathrm{t},{ }^{3} J_{C-F}=2.3 \mathrm{~Hz}\right), 33.2\left(\mathrm{dd},{ }^{2} J_{C-F}=25.1 \mathrm{~Hz},{ }^{2} J_{C-F}=\right.$ $22.4 \mathrm{~Hz}), 30.1\left(\mathrm{~d},{ }^{4} J_{C-F}=1.6 \mathrm{~Hz}\right), 19.3\left(\mathrm{dd},{ }^{3} J_{C-F}=10.2 \mathrm{~Hz},{ }^{3} J_{C-F}=0.5 \mathrm{~Hz}\right)$. ${ }^{19} \mathrm{~F}\{\mathrm{H}\} \mathrm{NMR}\left(471 \mathrm{MHz}, \mathrm{CDCl}_{3}\right) \delta-95.60\left(\mathbf{A B}\right.$ system, $\left.{ }^{2} J_{F-F}=246.2 \mathrm{~Hz}\right)$, 108.21 (AB system, ${ }^{2} J_{F-F}=246.6 \mathrm{~Hz}$ ). HRMS (ESI): calcd. for $[\mathrm{M}+\mathrm{Na}]^{+}$ $\left(\mathrm{C}_{15} \mathrm{H}_{17} \mathrm{NO}_{4} \mathrm{~F}_{2} \mathrm{Na}\right)=336.1018$; found: 336.1022 (1 ppm).

\section{Synthesis of 4,4-difluoro-2-phenyl-3a,4,5,6,7,7a-hexahydro-3H-indole} 1-oxide 26a

According to the general procedure 3 . White solid, yield $37 \mathrm{mg}$ (56\%) from $75 \mathrm{mg}(0.26 \mathrm{mmol})$ of $25 \mathrm{a}$. $\mathrm{R}_{\mathrm{f}}=0.2$ (Hexane / Ethyl Acetate: $\left.4 / 6\right) . \mathrm{mp}=$ $206{ }^{\circ} \mathrm{C} .{ }^{1} \mathrm{H}$ NMR $\left(300 \mathrm{MHz}, \mathrm{CDCl}_{3}\right) \delta 8.38-8.18(\mathrm{~m}, 2 \mathrm{H}), 7.52-7.33(\mathrm{~m}$, $3 \mathrm{H}), 3.97\left(\mathrm{t},{ }^{3} \mathrm{JH}_{\mathrm{H}}=11.9 \mathrm{~Hz}, 1 \mathrm{H}\right), 3.23-3.01(\mathrm{~m}, 2 \mathrm{H}), 2.64-2.49(\mathrm{~m}, 1 \mathrm{H})$, $2.45-2.17(\mathrm{~m}, 2 \mathrm{H}), 2.16-1.45(\mathrm{~m}, 4 \mathrm{H}) .{ }^{13} \mathrm{C} \mathrm{NMR}\left(75 \mathrm{MHz}, \mathrm{CDCl}_{3}\right) \delta$ $140.1,130.5,129.3,128.7,127.2,122.3\left(\mathrm{dd},{ }^{1} J_{C-F}=246.9 \mathrm{~Hz},{ }^{1} J_{C-F}=245.3\right.$ $\mathrm{Hz}), 73.4\left(\mathrm{~d},{ }^{3} J_{C-F}=9.0 \mathrm{~Hz}\right), 46.9\left(\mathrm{dd},{ }^{2} J_{C-F}=25.5 \mathrm{~Hz},{ }^{2} J_{C-F}=22.6 \mathrm{~Hz}\right), 34.2$ $\left(\mathrm{dd},{ }^{2} J_{C-F}=25.0 \mathrm{~Hz},{ }^{2} J_{C-F}=21.9 \mathrm{~Hz}\right), 27.8,26.5\left(\mathrm{~d},{ }^{4} J_{C-F}=1.6 \mathrm{~Hz}\right), 21.2(\mathrm{~d}$, $\left.{ }^{3} J_{C-F}=9.5 \mathrm{~Hz}\right) .{ }^{19} \mathrm{~F}\{\mathrm{H}\} \mathrm{NMR}\left(282 \mathrm{MHz}, \mathrm{CDCl}_{3}\right) \delta-99.88$ (AB system, ${ }^{2} J_{F-F}$ $=234.0 \mathrm{~Hz}),-113.99\left(\mathrm{AB}\right.$ system, $\left.{ }^{2} \mathrm{~J} F-F=234.0 \mathrm{~Hz}\right)$. HRMS (ESI): calcd. for $[\mathrm{M}+\mathrm{Na}]^{+}\left(\mathrm{C}_{14} \mathrm{H}_{15} \mathrm{NOF}_{2} \mathrm{Na}\right)=274.1013$; found: $274.1013(0 \mathrm{ppm})$.

Synthesis of 4,4-difluoro-2-(4-fluorophenyl)-3a,4,5,6,7,7a-hexahydro$3 \mathrm{H}$-indole 1-oxide 26b

According to the general procedure 3. Pale yellow solid, yield $47 \mathrm{mg}$ (70\%) from $75 \mathrm{mg}(0.25 \mathrm{mmol})$ of 25b. $R_{\mathrm{f}}=0.1$ (Hexane / Ethyl Acetate: $\left.4 / 6\right) . \mathrm{mp}$ $=216{ }^{\circ} \mathrm{C} .{ }^{1} \mathrm{H}$ NMR $\left(300 \mathrm{MHz}, \mathrm{CDCl}_{3}\right) \delta 8.41-8.27(\mathrm{~m}, 2 \mathrm{H}), 7.19-7.06$ $(\mathrm{m}, 2 \mathrm{H}), 3.95\left(\mathrm{t},{ }^{3} \mathrm{~J}_{\mathrm{H}-\mathrm{H}}=11.9 \mathrm{~Hz}, 1 \mathrm{H}\right), 3.24-2.92(\mathrm{~m}, 2 \mathrm{H}), 2.63-2.49(\mathrm{~m}$, $1 \mathrm{H}), 2.45-2.13(\mathrm{~m}, 2 \mathrm{H}), 2.10-1.43(\mathrm{~m}, 4 \mathrm{H}) .{ }^{13} \mathrm{C} \mathrm{NMR}\left(75 \mathrm{MHz}, \mathrm{CDCl}_{3}\right)$ $\delta 163.4\left(\mathrm{~d},{ }^{1} J_{C-F}=252.8 \mathrm{~Hz}\right), 139.1,129.5\left(\mathrm{~d},{ }^{3} J_{C-F}=8.3 \mathrm{~Hz}\right), 125.8\left(\mathrm{~d},{ }^{4} J_{C}-\right.$ $F=3.3 \mathrm{~Hz}), 122.3\left(\mathrm{dd},{ }^{1} J_{C-F}=246.9 \mathrm{~Hz},{ }^{1} J_{C-F}=245.4 \mathrm{~Hz}\right), 115.8\left(\mathrm{~d},{ }^{2} J_{C-F}=\right.$ $21.7 \mathrm{~Hz}), 73.3\left(\mathrm{~d},{ }^{3} J_{C-F}=8.9 \mathrm{~Hz}\right), 46.8\left(\mathrm{dd},{ }^{2} J_{C-F}=25.5 \mathrm{~Hz},{ }^{2} J_{C-F}=22.6 \mathrm{~Hz}\right)$, $34.2\left(\mathrm{dd},{ }^{2} J_{C-F}=25.0 \mathrm{~Hz},{ }^{2} J_{C-F}=21.9 \mathrm{~Hz}\right), 27.8,26.5\left(\mathrm{~d},{ }^{4} J_{C-F}=1.7 \mathrm{~Hz}\right)$, $21.1\left(\mathrm{~d},{ }^{3} J_{C-F}=9.6 \mathrm{~Hz}\right) .{ }^{19} \mathrm{~F}\{\mathrm{H}\} \mathrm{NMR}\left(282 \mathrm{MHz}, \mathrm{CDCl}_{3}\right) \delta-99.90(\mathrm{AB}$ system, ${ }^{2} J_{F-F}=234.4 \mathrm{~Hz}$ ), $-107.81(\mathrm{~s}),-114.08\left(\right.$ AB system, ${ }^{2} J_{F-F}=234.4$ $\mathrm{Hz})$. HRMS (ESI): calcd. for $[\mathrm{M}+\mathrm{Na}]^{+}\left(\mathrm{C}_{14} \mathrm{H}_{14} \mathrm{NOF}_{3} \mathrm{Na}\right)=292.0919$; found: 292.0918 (1 ppm).

Synthesis of 2-(4-chlorophenyl)-4,4-difluoro-3a,4,5,6,7,7a-hexahydro$3 \mathrm{H}$-indole 1-oxide 26c

According to the general procedure 3 . Yellow solid, yield $40 \mathrm{mg}(60 \%)$ from $75 \mathrm{mg}(0.24 \mathrm{mmol})$ of $25 \mathrm{c} . \mathrm{R}_{\mathrm{f}}=0.2$ (Hexane / Ethyl Acetate: $\left.4 / 6\right) . \mathrm{mp}=$ $208^{\circ} \mathrm{C} .{ }^{1} \mathrm{H}$ NMR $\left(300 \mathrm{MHz}, \mathrm{CDCl}_{3}\right) \delta 8.40-8.18(\mathrm{~m}, 2 \mathrm{H}), 7.47-7.30(\mathrm{~m}$, $2 \mathrm{H}), 3.95\left(\mathrm{t},{ }^{3} \mathrm{JH}_{\mathrm{H}}=12.0 \mathrm{~Hz}, 1 \mathrm{H}\right), 3.22-2.97(\mathrm{~m}, 2 \mathrm{H}), 2.64-2.49(\mathrm{~m}, 1 \mathrm{H})$, $2.43-1.97(\mathrm{~m}, 3 \mathrm{H}), 1.90-1.46(\mathrm{~m}, 3 \mathrm{H}) .{ }^{13} \mathrm{C} \mathrm{NMR}\left(75 \mathrm{MHz}, \mathrm{CDCl}_{3}\right) \delta$ $139.0,136.0,128.9,128.4,127.8,122.2\left(\mathrm{dd},{ }^{1} J_{C-F}=246.9 \mathrm{~Hz},{ }^{1} J_{C-F}=245.4\right.$ $\mathrm{Hz}), 73.5\left(\mathrm{~d},{ }^{3} J_{C-F}=9.0 \mathrm{~Hz}\right), 46.8\left(\mathrm{dd},{ }^{2} J_{C-F}=25.5 \mathrm{~Hz},{ }^{2} J_{C-F}=22.6 \mathrm{~Hz}\right), 34.2$ $\left(\mathrm{dd},{ }^{2} J_{C-F}=25.0 \mathrm{~Hz},{ }^{2} J_{C-F}=21.9 \mathrm{~Hz}\right), 27.6,26.4\left(\mathrm{~d},{ }^{4} J_{C-F}=1.6 \mathrm{~Hz}\right), 21.1(\mathrm{~d}$, $\left.{ }^{3} J_{C-F}=9.5 \mathrm{~Hz}\right) .{ }^{19} \mathrm{~F}\{\mathrm{H}\} \mathrm{NMR}\left(282 \mathrm{MHz}, \mathrm{CDCl}_{3}\right) \delta-99.90$ (AB system, ${ }^{2} J_{F-F}$ $=234.2 \mathrm{~Hz}$ ), -113.97 (AB system, ${ }^{2} J_{F-F}=234.2 \mathrm{~Hz}$ ). HRMS (ESI): calcd. for $[\mathrm{M}+\mathrm{Na}]^{+}\left(\mathrm{C}_{14} \mathrm{H}_{14} \mathrm{NOF}_{2}{ }^{35} \mathrm{CINa}\right)=308.0624$; found: 308.0627 (1 ppm).

Synthesis of 4,4-difluoro-2-(furan-2-yl)-3a,4,5,6,7,7a-hexahydro-3Hindole 1-oxide 26d

According to the general procedure 3 . Yellow solid, yield $53 \mathrm{mg}(80 \%)$ from $75 \mathrm{mg}\left(0.27 \mathrm{mmol}\right.$ ) of $25 \mathrm{~d}$. $R_{\mathrm{f}}=0.1$ (Hexane / Ethyl Acetate: 4/6). $\mathrm{mp}=$ $213^{\circ} \mathrm{C} .{ }^{1} \mathrm{H} \mathrm{NMR}\left(300 \mathrm{MHz}, \mathrm{CDCl}_{3}\right) \delta 7.78\left(\mathrm{~d},{ }^{3} \mathrm{H}-\mathrm{H}=3.5 \mathrm{~Hz}, 1 \mathrm{H}\right), 7.49(\mathrm{~d}$, $\left.{ }^{3} J_{H-H}=1.7 \mathrm{~Hz}, 1 \mathrm{H}\right), 6.58\left(\mathrm{dd},{ }^{3} J_{H-H}=3.5 \mathrm{~Hz},{ }^{3} J_{H-H}=1.8 \mathrm{~Hz}, 1 \mathrm{H}\right), 3.88(\mathrm{t}$, $\left.{ }^{3} J_{H-H}=12.1 \mathrm{~Hz}, 1 \mathrm{H}\right), 3.21\left(\mathrm{dd},{ }^{2} J_{H-H}=16.1 \mathrm{~Hz},{ }^{3} J_{H-H}=6.6 \mathrm{~Hz}, 1 \mathrm{H}\right), 3.03$ (ddd, $\left.{ }^{2} \mathrm{~J}_{\mathrm{H}-\mathrm{H}}=16.1 \mathrm{~Hz},{ }^{3} \mathrm{JH}_{\mathrm{H}-\mathrm{H}}=11.1 \mathrm{~Hz},{ }^{4} \mathrm{~J}_{\mathrm{H}-\mathrm{F}}=2.8 \mathrm{~Hz}, 1 \mathrm{H}\right), 2.62-2.48(\mathrm{~m}$, $1 \mathrm{H}), 2.46-2.00(\mathrm{~m}, 3 \mathrm{H}), 1.94-1.40(\mathrm{~m}, 3 \mathrm{H}) .{ }^{13} \mathrm{C} \mathrm{NMR}\left(75 \mathrm{MHz}, \mathrm{CDCl}_{3}\right)$ $\delta$ 145.4, 143.7, 133.7, $122.2\left(\mathrm{dd},{ }^{1} J_{C-F}=247.0 \mathrm{~Hz},{ }^{1} J_{C-F}=245.4 \mathrm{~Hz}\right), 114.7$, 112.7, $72.0\left(\mathrm{~d},{ }^{3} J_{C-F}=9.1 \mathrm{~Hz}\right), 47.6\left(\mathrm{dd},{ }^{2} J_{C-F}=25.5 \mathrm{~Hz},{ }^{2} J_{C-F}=22.6 \mathrm{~Hz}\right)$, $34.2\left(\mathrm{dd},{ }^{2} J_{C-F}=25.1 \mathrm{~Hz},{ }^{2} J_{C-F}=21.9 \mathrm{~Hz}\right), 26.3\left(\mathrm{~d},{ }^{3} J_{C-F}=1.6 \mathrm{~Hz}\right), 26.0(\mathrm{~d}$, 
$\left.{ }^{4} J_{C-F}=1.0 \mathrm{~Hz}\right), 21.2\left(\mathrm{~d},{ }^{3} J_{C-F}=9.6 \mathrm{~Hz}\right) .{ }^{19} \mathrm{~F}\{\mathrm{H}\} \mathrm{NMR}\left(282 \mathrm{MHz}, \mathrm{CDCl}_{3}\right) \delta-$ 99.8 (AB system, $\left.{ }^{2} J_{F-F}=234.7 \mathrm{~Hz}\right),-113.7\left(\mathrm{AB}\right.$ system, $\left.{ }^{2} J_{F-F}=234.7 \mathrm{~Hz}\right)$. HRMS (ESI): calcd. for $[\mathrm{M}+\mathrm{Na}]^{+}\left(\mathrm{C}_{12} \mathrm{H}_{13} \mathrm{NO}_{2} \mathrm{~F}_{2} \mathrm{Na}\right)=264.0807$; found: 264.0810 (1 ppm).

\section{Synthesis of 4,4-difluoro-2-(4-methoxyphenyl)-3a,4,5,6,7,7a- hexahydro-3H-indole 1-oxide $26 \mathrm{e}$}

According to the general procedure 3 . White solid, yield $51 \mathrm{mg}(75 \%)$ from $75 \mathrm{mg}(0.24 \mathrm{mmol})$ of $25 \mathrm{e} . \mathrm{R}_{\mathrm{f}}=0.1$ (Petroleum Ether / Ethyl Acetate: $5 / 5$ ). $\mathrm{mp}=201^{\circ} \mathrm{C} .{ }^{1} \mathrm{H}$ NMR $\left(500 \mathrm{MHz}, \mathrm{CDCl}_{3}\right) \delta 8.44-8.25(\mathrm{~m}, 2 \mathrm{H}), 7.06-$ $6.78(\mathrm{~m}, 2 \mathrm{H}), 4.05-3.78(\mathrm{~m}, 1 \mathrm{H}), 3.86(\mathrm{~s}, 3 \mathrm{H}), 3.33-2.97(\mathrm{~m}, 2 \mathrm{H}), 2.75$ $-2.48(\mathrm{~m}, 1 \mathrm{H}), 2.40-2.03(\mathrm{~m}, 3 \mathrm{H}), 1.92-1.49(\mathrm{~m}, 3 \mathrm{H}) .{ }^{13} \mathrm{C}$ NMR $(126$ $\left.\mathrm{MHz}, \mathrm{CDCl}_{3}\right) \delta 161.3,140.8,129.4,127.4,122.4\left(\mathrm{t},{ }^{1} J_{C-F}=246.2 \mathrm{~Hz}\right)$, $114.0,73.1,55.5,47.0\left(\mathrm{t},{ }^{2} J_{C-F}=23.8 \mathrm{~Hz}\right), 34.3\left(\mathrm{dd},{ }^{2} J_{C-F}=25.2 \mathrm{~Hz},{ }^{2} J_{C-F}=\right.$ $21.8 \mathrm{~Hz}), 27.7,26.6,21.2\left(\mathrm{~d},{ }^{3} \mathrm{~J}_{\mathrm{C}-\mathrm{F}}=9.5 \mathrm{~Hz}\right) .{ }^{19} \mathrm{~F}\{\mathrm{H}\} \mathrm{NMR}(471 \mathrm{MHz}$, $\left.\mathrm{CDCl}_{3}\right) \delta-99.85\left(\mathrm{AB}\right.$ system, $\left.{ }^{2} J_{F-F}=234.0 \mathrm{~Hz}\right),-113.96$ (AB system, ${ }^{2} J_{F-F}$ $=234.0 \mathrm{~Hz})$. HRMS (ESI): calcd. for $[\mathrm{M}+\mathrm{Na}]^{+}\left(\mathrm{C}_{15} \mathrm{H}_{17} \mathrm{NO}_{2} \mathrm{~F}_{2} \mathrm{Na}\right)=$ 304.1120; found: 304.1123 (1 ppm).

\section{Acknowledgements}

This research has been supported in Lebanon by the Research Grant program at Lebanese University. In France it has been supported by French CNRS and University of Rennes 1 (CNRS UMR 6226). We thank European FEDER founds for acquisition of D8Venture X-ray diffractometer used for crystal structure determination.

The authors would like to acknowledge the National Council for Scientific Research of Lebanon (CNRS-L)/Agence Universitaire de la Francophonie (AUF)/and the Lebanese University (UL) for granting a doctoral fellowship to Ali Soulieman. We are very grateful to the Centre Régional de Mesures Physiques de l'Ouest, Rennes (CRMPO) team for the physicochemical analyses and to Dr F. Justaud for fruitful discussions.

Keywords: fluorine $\cdot$ nitrones $\cdot$ cascade reactions $\bullet$ propargylic fluorides $\cdot$ nitroalkanes

[1] a) D. Becker, Cell. Mol. Life Sci. 1999, 56, 626-633; b) D. Becker, J. Am. Chem. Soc. 1996, 118, 905-906; c) D. Becker, J. Ley, L. Echegoyen, R. Alvarado, J. Am. Chem. Soc. 2002, 124, 4678-4684.

[2] a) M. Rosselin, F. Choteau, K.Zéamari, K. M. Nash, A. Das, R. Lauricella, E. Lojou, B.Tuccio, F. A. Villamena, G. Durand, J. Org. Chem. 2014, 79, 6615-6626; b) M. Rosselin, B. Tuccio, P. Pério, F. A. Villamena, P.-L. Fabre, G.Durand, Electrochimica Acta 2016, 193, 231-239; c) A. Deletraz, K. Zéamari, F. Di Meo, P.-L. Fabre, K. Reybier, P. Trouillas, B. Tuccio, G. Durand, New J. Chem. 2019, 43, 15754-15762.

[3] F. A. Villamena, J. L. Zweier, Antiox. Redox Signal. 2004, 6, 619-629.

[4] G. I. Likhtenshtein, In Nitroxides: Brief History, Fundamentals, and Recent Developments; Likhtenshtein, G. I., Ed.; Springer International Publishing: Cham, 2020.

[5] R. A. Floyd, H. C. C. F. Neto, G. A. Zimmerman, K. Hensley, R. A. Towner, Free Radical Biology and Medicine 2013, 62, 145.

[6] (a) H. Karoui, C. Nsanzumuhire, F. Le Moigne, M. Hardy, D. Siri, E. Derat, A. Rockenbauer, O. Ouari, P. Tordo, Chem. Eur. J. 2014, 20, 4064-4071; (b) A. Deletraz, K. Zeamari, K. Hua, M. Combes, F. A. Villamena, B. Tuccio, N. Callizot, G. Durand, J. Org. Chem. 2020, 85, 6073-6085; (c) D. G. Piotrowska, L. Mediavilla, L. Cuarental, I. E. Glowacka, J. MarcelloContelles, D. Hadjipavlou-Litina, F. Lopez-Munoz, M. J. Oset-Gasque, ACS Omega, 2019, 4, 8581-8587; (d) S. Cancella, L. Canclini, G. Mourglia-Ettlin, P. Hernandez, A. Merlino, Eur. J. Pharmacol. 2020, 871, 172926.

[7] L. Belayev, D. Becker, O. Alonso, Y. Liu, R. Busto, J. Ley, M. Ginsberg, J. Neurosurg. 2002, 96, 1077-1083.
[8] B. Guo, D. Xu, H. Duan, J. Du, Z. Zhang, S. Lee, Y. Wang, Biol. Pharm. Bull. 2014, b13-00743.

[9] X. Cao, J. W. Phillis, Brain Res.1994, 644, 267-272

[10] M. Ginsberg, D. Becker, R. Busto, A. Belayev, Y. Zhang, L. Khoutorova, J. Ley, W. Zhao, L. Belayev, Ann. Neurol., 2003, 54, 330-342.

[11] Q. Zhao, K. Pahlmark, M. Smith, B. Siesjö, Acta Physiol. Scand. 1994, 152, 349-350

[12] R. Floyd, H. Chandru, T. He, R. Towner, Anti-Cancer Agents in Medicinal Chemistry (Formerly Current Medicinal Chemistry-Anti-Cancer Agents), 2011, 11, 373-379

[13] R. Floyd, FASEB J., 1990, 4, 2587-2597.

[14] T. He, S. Doblas, D. Saunders, R. Casteel, M. Lerner, J. Ritchey, T. Snider, R. Floyd, R. Towner, Free Rad. Biol. Med. 2011, 51, 490-502.

[15] D. Nakae, H. Kishida, T. Enami, Y. Konishi, K. Hensley, R. Floyd, Y. Kotake, Cancer Science, 2003, 94, 26-31.

[16] S. Murahashi, Y. Imada, Chem. Rev. 2019, 119, 4684-4716.

[17] For selected reviews on this topic, see a) S. Purser, P. R. Moore, S. Swallow, V. Gouverneur, Chem. Soc. Rev. 2008, 37, 320-330; b) E. P. Gillis, K. J. Eastman, M. D. Hill, D. J. Donnelly, N. A. Meanwell, J. Med. Chem. 2015, 58, 8315-8359; c) P. A. Champagne, J. Desroches, J.-D. Hamel, M. Vandamme, J.-F. Paquin, Chem. Rev. 2015, 115, 9073-9174; d)Y. Zhou, J. Wang, Z. Gu, S. Wang, W. Zhu, J. L. Aceňa, V. A. Soloshonok, K. Izawa, H. Liu, Chem. Rev. 2016, 116, 422-518; e) D. E. Yerien, S. Bonesi, A. Postigo, Org. Biomol. Chem. 2016, 14, 8398-8427; f) N. A. Meanwell, J. Med. Chem. 2018, 61, 5822-5880; M. Inoue, Y. Sumii, N. Shibata, ACS Omega 2020, 5, 10633-10640 and references cited therein.

[18] B. S. Selinsky, L. A. Levy, A. G. Motten, R. E. London J. Magn. Res. 1989, 81, 57-67.

[19] a) G. Mloston, E. Obijalska, M. Celeda, V. Mittermeier, A. Linden, H. Heimgartner, J. Fluor. Chem. 2014, 165, 27-32; b) Q. Zeng, L. Zhang, J Yang, B. Xu, Y. Xiao, J. Zhang, Chem. Comm. 2014, 50, 4203-4206; c) H. Kawai, Z. Yuan, T. Kitayama, E. Tokunaga, N. Shibata, Ang. Chem. Inter. Edit. 2013, 52, 5575-5579; d) S. Xu, Z.-M. Zhang, B. Xu, B. Liu, Y. Liu, J. Zhang, J. Am. Chem. Soc. 2018, 140, 2272-2283.

[20] For representative examples of various uses of ${ }^{19} \mathrm{~F} \mathrm{NMR}$ in bioorganic and medicinal chemistry, see : a) T. Didenko, J. J. Liu, R. Horst, R. C. Stevens, K. Wüthrich, Current Opinion in Structural Biology, 2013, 23 740-747; b) H. Chen, S. Viel, F. Ziarelli, L. Peng, Chem. Soc. Rev., 2013 42, 7971-7982; c) E. Marsh, G. Neil, Y. Suzuki, ACS Chem. Biol. 2014, 9, $1242-1250$; d) I. Tirotta, V. Dichiarante, C. Pigliacelli, G. Cavallo, G. Terraneo, F. B. Bombelli, P. Metrangolo, G. Resnati, Chem. Rev., 2015, 115, 1106-1129; e) K. E. Arntson, W. C. K. Pomerantz, J. Med. Chem. 2016, 59, 5158-5171; f) T. Sugiki, K. Furuita, T. Fujiwara, C. Kojima, Molecules, 2018, 23, 148/1-148/27; g) C. Dalvit, A. Vulpetti, J. Med.Chem. 2019, 62, 2218-2244; h) C. B. Kang, Current Medicinal Chemistry, 2019, 26, 4964-4983; i) D. Xie, M. Yu, R. T. Kadakia, E. L. Que, Acc. Chem. Res. 2020, 53, 2-10; j) J. N. Frei, R. W. Broadhurst, M. J. Bostock, A. Solt, A. J. Jones, F. Gabriel, A. Tandale, B. Shrestha, D. Nietlispach, Nature Commun. 2020, 11, 1-14.

[21] For a review see: A. Hachem, D. Grée, S. Chandrasekhar, R. Grée, Synthesis 2017, 49, 2101-2116 and references cited therein.

[22] L. Hariss, R. Ibrahim, N. Jaber, T. Roisnel, R. Grée, A. Hachem Eur. J. Org. Chem. 2018, 3782-3791

[23] L. Hariss, K. B. Hadir, M. El-Masri, T. Roisnel, R. Grée, A. Hachem, Beilstein J. Org. Chem. 2017, 13, 2115-2121.

[24] CCDC 1990242 (for compound 6e) and CCDC 1990243 (for compound 26a) and CCDC 1990244 (for compound 26b) contain the supplementary crystallographical data for this paper. These data can be obtained free of charge from the Cambridge Crystallographic Data Centre.

[25] Y. Kiyotsuka, J. Igarashi, Y. Kobayashi, Tetrahedron Lett. 2002, 43 2725-2729.

[26] A closely related process has been observed already in the synthesis of sulfur-containing heterocycles, see ref [22]. 


\section{Entry for the Table of Contents}

Insert graphic for Table of Contents here.

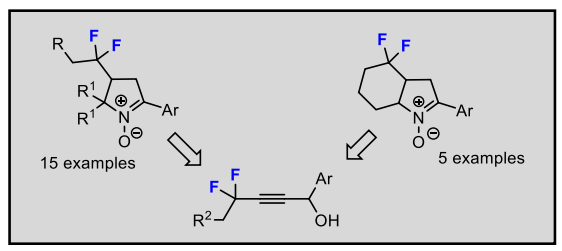

New mono- and bicyclic nitrones containing a $\mathrm{CF}_{2}$ group have been efficiently synthetized starting from propargylic fluorides. The key steps involve isomerization of propargylic alcohols to enones, followed by nucleophilic addition of nitro alkane anions and then intramolecular Michael additions. The last step is a reductive cyclisation from the nitro group. 